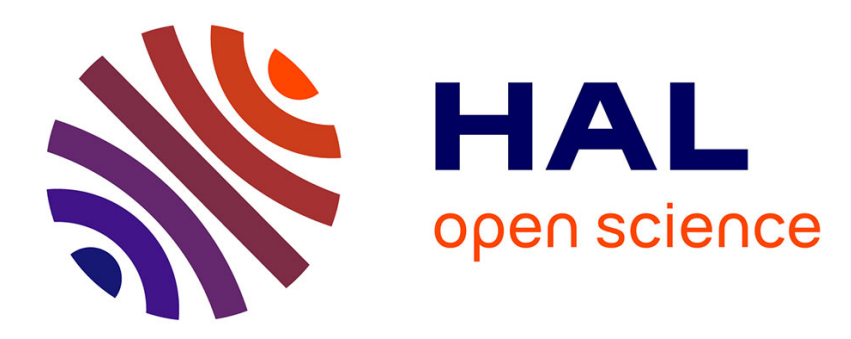

\title{
Testing for Spatial Autocorrelation in a Fixed Effects Panel Data Model
}

\author{
Nicolas Debarsy, Cem Ertur
}

\section{To cite this version:}

Nicolas Debarsy, Cem Ertur. Testing for Spatial Autocorrelation in a Fixed Effects Panel Data Model. Regional Science and Urban Economics, 2010, 40, pp.453-470. halshs-00414133

\section{HAL Id: halshs-00414133 \\ https://shs.hal.science/halshs-00414133}

Submitted on 28 Sep 2009

HAL is a multi-disciplinary open access archive for the deposit and dissemination of scientific research documents, whether they are published or not. The documents may come from teaching and research institutions in France or abroad, or from public or private research centers.
L'archive ouverte pluridisciplinaire HAL, est destinée au dépôt et à la diffusion de documents scientifiques de niveau recherche, publiés ou non, émanant des établissements d'enseignement et de recherche français ou étrangers, des laboratoires publics ou privés. 


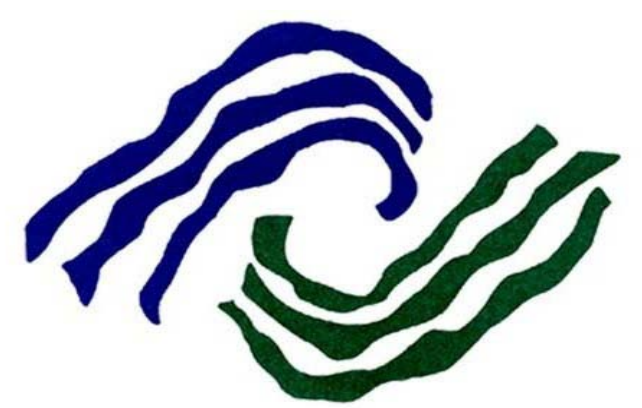

Laboratoire d'Economie d'Orléans

\title{
Document de Recherche
}

$n^{\circ} 2009-12$

\section{«Testing for Spatial Autocorrelation in a fixed Effects Panel Data Model »}

\author{
Nicolas DEBARSY \\ Cem ERTUR
}

Laboratoire d'Economie d'Orléans - UMR CNRS 6221 Faculté de Droit, d'Economie et de Gestion, Rue de Blois, B.P. 6739 - 45067 Orléans Cedex 2 - France

Tél : 33 (0)2 38417037 - 33 (0)2 38494819 - Fax : 33 (0)2 38417380

E-mail : leo@univ-orleans.fr - http://www.univ-orleans.fr/DEG/LEO 


\title{
Testing for spatial autocorrelation in a fixed effects panel data model
}

\author{
Nicolas Debarsy* and Cem Ertur ${ }^{\dagger}$
}

July 2009

\begin{abstract}
This paper derives several Lagrange Multiplier statistics and the corresponding likelihood ratio statistics to test for spatial autocorrelation in a fixed effects panel data model. These tests allow discriminating between the two main types of spatial autocorrelation which are relevant in empirical applications, namely endogenous spatial lag versus spatially autocorrelated errors. In this paper, five different statistics are suggested. The first one, the joint test, detects the presence of spatial autocorrelation whatever its type. Hence, it indicates whether specific econometric estimation methods should be implemented to account for the spatial dimension. In case they need to be implemented, the other four tests support the choice between the different specifications, i.e. endogenous spatial lag, spatially autocorrelated errors or both. The first two are simple hypothesis tests as they detect one kind of spatial autocorrelation assuming the other one is absent. The last two take into account the presence of one type of spatial autocorrelation when testing for the presence of the other one. We use the methodology developed in Lee and $\mathrm{Yu}$ (2008) to set up and estimate the general likelihood function. Monte Carlo experiments show the good performance of our tests. Finally, as an illustration, they are applied to the Feldstein-Horioka puzzle. They indicate a misspecification of the investment-saving regression due to the omission of spatial autocorrelation. The traditional saving-retention coefficient is shown to be upward biased. In contrast our results favor capital mobility.

JEL Classification: C12, C21, C23

Keywords: Spatial Autocorrelation; Panel Data; Hypothesis Tests; FeldsteinHorioka
\end{abstract}

\footnotetext{
${ }^{*}$ Doctoral Researcher of the FNRS, PAI, CERPE, University of Namur.

${ }^{\dagger}$ Laboratoire d'Economie d'Orléans (LEO - UMR 6221 CNRS), Université d'Orléans.
} 


\section{Résumé}

Cette article propose de tester la présence d'autocorrélation spatiale dans un modèle de données de panel à effets fixes. Différentes statistiques du multiplicateur de Lagrange ainsi que les statistiques correspondantes du rapport de vraisemblance sont présentées. Ces tests permettent de distinguer les deux formes principales d'autocorrélation spatiales qui sont d'intérêt dans les applications empiriques, celle fondée sur la présence d'une variable endogène spatialement décalée et celle fondée sur un processus d'erreurs spatialement autocorrélées. Cinq statistiques de test sont ainsi développées. La première correspond au test d'une hypothèse nulle jointe et permet de détecter la présence d'autocorrélation spatiale quelque soit sa forme. Elle indique ainsi si des techniques économétriques spécifiques doivent être implémentées pour tenir compte de la dimension spatiale. Dans l'affirmative, les quatres autres statistiques de test permettent de choisir la spécification économétrique appropriée : le modèle avec variable endogène spatialement décalée, le modèle avec erreurs spatialement autocorrélées ou le modèle intégrant à la fois une variable endogène spatialement décalée et des erreurs spatialement autocorrélées. Les deux premières correspondent au test d'une hypothèse nulle simple dans la mesure où elles permettent de détecter une forme d'autocorrélation spatiale en supposant l'absence de la forme alternative, tandis que les deux dernières permettent de détecter la présence d'une forme d'autocorrélation spatiale en présence de la forme alternative. La méthodologie d'estimation par le maximum de vraisemblance développée par Lee et Yu (2008) est utilisée. Des simulations de Monte Carlo montrent la bonne performance des tests proposés. Finalement, une illustration empirique fondée sur le paradoxe de Feldstein-Horioka est présentée. Les tests développés indiquent la mauvaise spécification du modèle due à l'omission de l'autocorrélation spatiale entraînant un biais positif du coefficient de rétention de l'épargne traditionnellement obtenu dans la littérature. Nos résultats suggèrent une mobilité du capital plus importante.

Classification JEL : C12, C21, C23

Mots-clés : Autocorrelation spatiale; Données de panel; Tests d'hypothèse; Feldstein-Horioka 


\section{Introduction}

In the past decade, spatial econometric models designed to deal with spatial autocorrelation and heterogeneity have been mainly developed within the framework of cross-sectional data (Anselin, 1988a; Anselin and Bera 1998; Anselin, 2006). Estimation methods and hypothesis tests have been extensively studied in the econometric literature. Lee (2004) presents a comprehensive investigation of the asymptotic properties of the maximum likelihood estimators widely used in the literature to estimate spatial models. Kelejian and Prucha $(1998,1999)$ and Conley $(1999)$ propose generalized method of moments as an alternative estimation method. Various test statistics, mainly based on the Lagrange Multiplier (LM) principle, have been elaborated in Anselin et al. (1996) and Anselin (2001). Those spatial econometric tools have been successfully applied in the economic literature to highlight significant spatial effects in many fields such as growth economics ${ }^{1}$ (Conley and Ligon, 2002; Ertur and Koch, 2007), international economics (Baltagi et al., 2007b, 2008), labor economics (Conley and Toppa, 2002), public economics (Brueckner, 1998, 2003) agricultural and environmental economics (Anselin et al. 2004; Bell and Bockstael, 2000; Murdoch et al., 1997) etc. It is now widely known that ignoring spatial effects when they are actually present leads, at best, to inefficient OLS estimators and biased statistical inference and, at worst, to biased and inconsistent OLS estimators. ${ }^{2}$

Although Anselin (1988a, chapter 10, p.137-168) also proposed an early study of spatial panel data models, it is only recently that an increasing interest is emerging in the econometric literature calling into question the typical cross-sectional independence assumption in panel data models (Elhorst, 2003 ; Anselin et al., 2008 ; Yu et al. 2008 ; Lee and Yu, 2008, 2009). Panel data models are primarily designed to deal with individual heterogeneity, which can of course be inherently spatial, but not with individual interactions or spatial autocorrelation. In other words, in the fixed effects framework, heterogeneity due to individual characteristics, for instance "absolute" geographical localization, is easily taken into account by demeaning. However heterogeneity due to differentiated feedback effects, originated in cross-section interactions, based, for instance, on "relative" geographical localization of individuals with respect to each other, cannot be dealt with and requires explicit modeling of spatial autocorrelation. We label the latter type of heterogeneity interactive heterogeneity, genuinely spatial by nature, to avoid confusion with what is traditionally called spatial heterogeneity in the literature, which is actually standard individual heterogeneity coming from spatial structural instability in coefficients or residual variance. Spatial panel data models are exactly designed to deal with both type of heterogeneity: pure individual heterogeneity captured by fixed effects and interactive heterogeneity captured by impact coefficients or elasticities computed from the reduced form of the spatial autoregressive model taking into account the interaction structure between individuals.

To the best of our knowledge, Baltagi et al. (2003) are the first to propose to the applied researcher a joint test and two conditional LM tests as well as the corresponding likelihood ratio tests (LR) for the spatial error component model. The joint statistic simultaneously tests for the existence of spatial error correlation and random individual effects. The conditional statistics test for the existence of spatial error correlation assuming the presence of random individual effects or for the exis-

1. See Rey and Le Gallo (2009) for a recent survey.

2. LeSage and Pace (2009) present the state of the art in spatial econometric techniques. 
tence of random individual effects assuming the presence of spatial error correlation. Baltagi et al. (2007a) generalize these tests by deriving new test statistics that also consider the problem of serial correlation in the remainder error term. Finally, Baltagi and Liu (2008) allow for autoregressive spatial lag dependence in the dependent variable rather than the error term.

Other strands of the literature focus on nonparametric covariance matrix estimation techniques as Driscoll and Kray (1998) or on the residual multifactor approach, which assumes that the cross dependence can be characterized by a finite number of unobserved common factors, possibly due to economy-wide shocks that affect all units albeit with different intensities. Under this framework, the error term is a linear combination of few common time-specific effects with heterogeneous factor loadings plus an idiosyncratic (individual-specific) error term (Pesaran, 2006) and the model is estimated using the Common Correlated Effects (CCE) estimator. Furthermore Pesaran and Tosetti (2009) and Chudik et al. (2009) extend this approach to spatial processes. Note also that Pesaran (2004) propose a general diagnostic test for cross-section dependance in panels known as CD tests.

However none of those tests are designed to discriminate between the two types of spatial autocorrelation that may be relevant in applied models, namely spatially autocorrelated errors versus spatially lagged endogenous variable. This is precisely the aim of this paper in the framework of fixed effects spatial panel data model.

To be more precise, let us consider the classical linear model for the time period $t: \mathbf{Y}_{t}=\mathbf{X}_{t} \beta+\mathbf{U}_{t}$, where $\mathbf{Y}_{t}$ represents the $n \times 1$ vector of the dependent variable for all individuals, $\mathbf{X}_{t}$ is the $n \times k$ matrix of covariates and $\mathbf{U}_{t}$ is the error term. The first type of spatial autocorrelation usually takes the form of a simple first order spatial autoregressive process on the error term: $\mathbf{U}_{t}=\lambda \mathbf{W} \mathbf{U}_{t}+\mathbf{V}_{t}$ where $\mathbf{W}$ is the $n \times n$ row-standardized interaction or spatial weight matrix, $\lambda$ represents the intensity of spatial correlation between residuals and $\mathbf{V}_{t}$ is a $n \times 1$ vector of well behaved disturbances. This model, labeled SEM, is characterized, in reduced form, by a global spatial diffusion of a random shock: $\mathbf{Y}_{t}=\mathbf{X}_{t} \beta+(\mathbf{I}-\lambda \mathbf{W})^{-1} \mathbf{V}_{t}$ The second type of spatial autocorrelation usually takes the form of the first order spatial autoregressive model, labeled SAR: $\mathbf{Y}_{t}=\rho \mathbf{W} \mathbf{Y}_{t}+\mathbf{X}_{t} \beta+\mathbf{U}_{t}$ where $\mathbf{W} \mathbf{Y}_{t}$ is the $n \times 1$ vector of the endogenous spatial lag variable representing the spatially weighted mean of neighboring $\mathbf{Y}_{t}$ values, $\rho$ is the spatial autoregressive parameter and $\mathbf{U}_{t}$ is a $n \times 1$ vector of well behaved disturbances. Needless to say that the latter type of modeling spatial dependence may be theoretically founded and may be derived from structural equations as shown, for instance, in Ertur and Koch $(2007,2008)$ in the framework of the neo-classical and Schumpeterian growth theories. Note also, that in contrast to the former model, the latter allows, in reduced form, for full interactive heterogeneity of the impact coefficients or elasticities by means of the so called global spatial multiplier effect in addition to the global spatial diffusion property of a random shock (Anselin, 2003): $\mathbf{Y}_{t}=(\mathbf{I}-\rho \mathbf{W})^{-1} \mathbf{X}_{t} \beta+(\mathbf{I}-\rho \mathbf{W})^{-1} \mathbf{U}_{t}$. Finally, it is also possible to consider a model with both types of spatial autocorrelation: $\mathbf{Y}_{t}=\rho \mathbf{W} \mathbf{Y}_{t}+\mathbf{X}_{t} \beta+\mathbf{U}_{t}$ where $\mathbf{U}_{t}$ itself follows of first order spatial autoregressive process: $\mathbf{U}_{t}=\lambda \mathbf{M} \mathbf{U}_{t}+\mathbf{V}_{t}$ where $\mathbf{M}$ is a spatial weight matrix, which is often identical to $\mathbf{W}$ in empirical settings. This general model, labeled $\operatorname{SARAR}(1,1)$, exhibits the same properties as the previous spatial autoregressive model in terms of interactive heterogeneity, although the global spatial diffusion process of a random shock is here more complex.

The aim of this paper is therefore to assess whether spatial autocorrelation is present in the sample considered and in the affirmative, to identify the most appro- 
priate spatial specification as this appears to be a crucial point from the modeling perspective of individual and interactive heterogeneity. Five different LM and the corresponding LR tests statistics are provided. The joint test detects the presence of spatial autocorrelation whatever its type. Hence, it indicates whether specific econometric estimation methods should be implemented to account for the spatial dimension. In the case they need to be implemented, the other four tests support the choice between the different spatial specifications, i.e. a model with an endogenous spatial lag variable, a spatially autocorrelated error process or both. The first two are simple hypothesis tests as they detect only one type of spatial autocorrelation assuming the absence of the other one. The last two account for the possible presence of one type of spatial autocorrelation when testing for the presence of the other one. We use the methodology recently developed in Lee and Yu (2008) to set up the likelihood function of the fixed effects spatial panel data model. Some Monte Carlo experiments show the good performance of our tests in various settings.

Finally, an empirical application of our tests is provided in the framework of the well-known Feldstein-Horioka puzzle based on the strong empirical evidence of high correlation between domestic saving and domestic investment rates (Feldstein and Horioka, 1980). Actually, using our tests, we find evidence of significant spatial autocorrelation in the form of an endogenous spatial lag variable in the fixed effects spatial panel data specification. This result has major implications: first from the econometric perspective, the usual within estimator is thus biased and inconsistent. Second, individual and interactive heterogeneity are therefore both of interest. To be more precise the reduced form of the specification should be used to compute the heterogeneous impact coefficients, which reflect both direct effects including own spillovers and indirect effects, i.e. spillovers. In other words, a change in the saving rate in one country, say country $i$, affects the investment rate of that country $i$, which in turn affects the investment rates of other countries, which then feed back to the investment rate of country $i$. Estimation of the SAR fixed effects panel data model shows a significant drop in the mean of the heterogeneous saving-retention coefficients, which reflect the direct effect including own-spillovers. Moreover, we find strong evidence in favor of indirect spillovers effects, which are all significant. Our econometric results favor therefore capital mobility in contrast to most of the previous results obtained in the literature. Furthermore, they thus underline the importance of properly testing for spatial autocorrelation and estimating the adequate spatial econometric specification when using fixed effects panel data models.

The remainder of the paper is organized as follows: section 2 presents the fixed effects spatial panel data model, the general framework used and the test statistics proposed. All derivations are relegated to the appendix. Section 3 presents the performance of the tests by using Monte Carlo simulations. Section 4 presents the application of our tests to the Feldstein-Horioka puzzle. The last section concludes.

\section{The model and the test statistics}

Our benchmark model, elaborated by Lee and Yu (2008), combines a spatial autoregressive model with spatially autocorrelated disturbances of order $(1,1)$, labeled SARAR(1,1), in a fixed effects spatial panel data setting:

$$
\begin{aligned}
\mathbf{Y}_{n, t} & =\rho \mathbf{W}_{n} \mathbf{Y}_{n, t}+\mathbf{X}_{n, t} \beta+\mu_{n}+\mathbf{U}_{n, t} \\
\mathbf{U}_{n, t} & =\lambda \mathbf{M}_{n} \mathbf{U}_{n, t}+\mathbf{V}_{n, t} \quad t=1, \ldots, T
\end{aligned}
$$


where $\mathbf{Y}_{n, t}=\left(y_{1, t}, y_{2, t}, \ldots, y_{n, t}\right)$ is the $n \times 1$ vector of the dependent variable for all individuals in period $t, \mathbf{X}_{n, t}$ is the $n \times k$ matrix of exogenous variables, $\beta$ is the associated vector of unknown regression coefficients to be estimated. $\mathbf{V}_{n, t}=$ $\left(v_{1, t}, v_{2, t}, \ldots, v_{n, t}\right)$ is the innovation term, $v_{i, t}$ is i.i.d. across $i$ and $t$ with zero mean and variance $\sigma^{2}$, and $\mu_{n}$ is the $n \times 1$ vector of individual fixed effects. $\mathbf{W}_{n}$ and $\mathbf{M}_{n}$ are $n \times n$ non-stochastic matrices typically referred to as interaction or spatial weight matrices, $\rho$ and $\lambda$ are the unknown spatial autoregressive parameters to be estimated. Our analysis also allows for $\mathbf{W}_{n}=\mathbf{M}_{n}$, which will be frequently the case in applications. We assume the same interaction pattern for all time periods (i.e. constant weight matrices over time). We consider fixed effects for individuals only and assume SAR errors in the idiosyncratic term. ${ }^{3}$ Finally, since we are interested in the spatial dimension of the model, the sample is arranged first by period and second by individual. ${ }^{4}$

The assumptions underlying the asymptotic properties of (quasi)-maximum likelihood estimators for the SARAR $(1,1)$ model are developed and discussed in $\mathrm{Yu}$ et al. (2008) and Lee and $\mathrm{Yu}$ (2008) in the panel data setting. Let us define $\mathbf{S}_{n}(\rho)=\left(\mathbf{I}_{n}-\rho \mathbf{W}_{n}\right)$ and $\mathbf{R}_{n}(\lambda)=\left(\mathbf{I}_{n}-\lambda \mathbf{M}_{n}\right)$. A restricted version of this set of assumptions for normal disturbances is presented below:

Assumption 1. $\mathbf{W}_{n}$ and $\mathbf{M}_{n}$ are non-negative and non stochastic interaction matrices and are fixed through time. Moreover, $w_{i i}=0$ and $m_{i i}=0$, for $i=1, \ldots, n .^{5}$ Finally, both matrices are assumed to be uniformly bounded in row and column sums in absolute value.

Assumption 2. $\mathbf{S}_{\mathbf{n}}(\rho)$ and $\mathbf{R}_{n}(\lambda)$ are invertible for all $\rho \in \mathbb{P}$ and $\lambda \in \Lambda$. Moreover $\mathbf{S}_{\mathbf{n}}(\rho)^{-1}$ and $\mathbf{R}_{n}(\lambda)^{-1}$ are also uniformly bounded in row and column sums in absolute value for all $\rho \in \mathbb{P}$ and $\lambda \in \Lambda$. Furthermore, $\mathbb{P}$ and $\Lambda$ are compact.

Assumption 3. The disturbances $v_{i, t}$ are normally, identically and independently distributed across $i$ and $t$ with zero mean and variance $\sigma^{2}$.

Assumption 4. The regressor matrix $\mathbf{X}$ has full column rank, and its elements are non stochastic and uniformly bounded in $n$ and $t$. The limit of $\frac{1}{n T} \sum_{t=1}^{T}\left(\mathbf{X}_{n, t}-\right.$ $\left.\overline{\mathbf{X}}_{n, T}\right)^{\prime}\left(\mathbf{X}_{n, t}-\overline{\mathbf{X}}_{n, T}\right)$ exists and is non singular, where $\overline{\mathbf{X}}_{i, T}=\frac{1}{T} \sum_{t=1}^{T} \mathbf{X}_{i, t}$ is the time average for individual $i$.

Without loss of generality, we assume that, $\mathbf{W}_{n}$ and $\mathbf{M}_{n}$ are row-normalized. According to Lee and $\mathrm{Yu}(2008, \mathrm{p} .4)$, it first allows to consider as parameter space for both $\rho$ and $\lambda$ a compact subset of $(-1,1) .{ }^{6}$ Secondly, referring to Anselin and Bera (1998, p.243): "it facilitates the interpretation of operations with the weight matrix as an averaging of neighbouring values."

Under this set of assumptions, for each period $t$, the model can then be rewritten as follows in reduced form:

$$
\mathbf{Y}_{n, t}=\mathbf{S}_{n}(\rho)^{-1} \mathbf{X}_{n, t} \beta+\mathbf{S}_{n}(\rho)^{-1} \mu_{n}+\mathbf{S}_{n}(\rho)^{-1} \mathbf{R}_{n}(\lambda)^{-1} \mathbf{V}_{n, t}
$$

3. Kapoor et al. (2007) suggest an alternative framework where both the error term and individual effects are assumed to be spatially autocorrelated.

4. In other words, $i$ is the fast moving index whereas $t$ is the slow moving one.

5. This normalization assumption implies some simplifications in the interpretation of spatial parameters since self-influence is excluded in practice, see Yu et al. (2008, p.120).

6 . If the spatial weight matrix, for any reason, is not row-standardized, we can find a reparametrization that ensures the compactness of the parameter space (see Kelejian and Prucha, 2008). 
The variance-covariance matrix of the error term $\mathbf{U}_{n, t}$ is then:

$$
E\left(\mathbf{U}_{n, t} \mathbf{U}_{n, t}^{\prime}\right)=\sigma^{2} \mathbf{S}_{n}(\rho)^{-1} \mathbf{R}_{n}(\lambda)^{-1}\left(\mathbf{R}_{n}(\lambda)^{-1}\right)^{\prime}\left(\mathbf{S}_{n}(\rho)^{-1}\right)^{\prime},
$$

with $\sigma^{2} \mathbf{I}_{n}$, the variance-covariance matrix of the homoskedastic normal multivariate disturbance $\mathbf{V}_{n, t}$.

We follow the methodology developed by Lee and Yu (2008) to formulate the likelihood function. These authors do not apply the typical within transformation to the data to wipe out the individual effects. They show that even though the direct (within) approach developed in Elhorst (2003) provides consistent $\rho$ and $\beta$ coefficients, the variance of the disturbances is not consistently estimated when $T$ is small but $n$ is large (see Lee and Yu, 2008, 2009). These authors exploit the properties of eigenvectors to transform data in such a way that individual effects still get removed but the variance of the transformed error term is consistently estimated. Let us recall that the within estimation applies the demeaning operator $\mathbf{J}_{T}=\left(\mathbf{I}_{T}-\right.$ $\left.\frac{1}{T} \iota_{T} \iota_{T}^{\prime}\right)$ to the original data set and estimates the transformed model. Lee and $\mathrm{Yu}$ (2008) define $\left[\mathbf{F}_{T, T-1} \frac{1}{\sqrt{T}} \iota_{T}\right]$ as the orthonormal matrix of the eigenvectors of the demeaning operator, with $\mathbf{F}_{T, T-1}$ the $T \times(T-1)$ matrix of eigenvectors of $\mathbf{J}_{T}$ corresponding to eigenvalues equal to 1 and $\iota_{T}$, a $T \times 1$ vector of ones. The suggested transformation applies the $\mathbf{F}$ matrix to the original data set:

$$
\left(\begin{array}{c}
\mathbf{Y}_{n, 1}^{*} \\
\vdots \\
\mathbf{Y}_{n, T-1}^{*}
\end{array}\right)=\left(\mathbf{F}_{T, T-1}^{\prime} \otimes \mathbf{I}_{n}\right)\left(\begin{array}{c}
\mathbf{Y}_{n, 1} \\
\vdots \\
\mathbf{Y}_{n, T}
\end{array}\right)
$$

Hence, the transformed sample is shrunk of one period of time. Our benchmark model thus becomes:

$$
\begin{aligned}
& \mathbf{Y}_{n, t}^{*}=\rho \mathbf{W}_{n} \mathbf{Y}_{n, t}^{*}+\mathbf{X}_{n, t}^{*} \beta+\mathbf{U}_{n, t}^{*} \\
& \mathbf{U}_{n, t}^{*}=\lambda \mathbf{M}_{n} \mathbf{U}_{n, t}^{*}+\mathbf{V}_{n, t}^{*} \quad t=1, \ldots, T-1 .
\end{aligned}
$$

Let us look how the variance-covariance matrix of the transformed disturbance, $\mathbf{V}_{n, t}^{*}$ is affected by this "pseudo-within" transformation. As $\mathbf{V}_{n, t}^{*}$ is a linear transformation of the original disturbance, its mean remains 0 and its variance-covariance matrix is:

$$
\begin{aligned}
E\left(\mathbf{V}^{*} \mathbf{V}^{* \prime}\right) & =\left(\begin{array}{c}
\mathbf{V}_{n, 1}^{*} \\
\vdots \\
\mathbf{V}_{n, T-1}^{*}
\end{array}\right)\left(\begin{array}{lll}
\mathbf{V}_{n, 1}^{*} & \ldots & \mathbf{V}_{n, T-1}^{*}
\end{array}\right) \\
& =\sigma^{2}\left(\mathbf{F}_{T, T-1}^{\prime} \otimes \mathbf{I}_{n}\right)\left(\mathbf{F}_{T, T-1} \otimes \mathbf{I}_{n}\right)=\sigma^{2} \mathbf{I}_{n(T-1)} .
\end{aligned}
$$

Two results can be highlighted: the variance-covariance matrix is well-defined and $v_{i, t}^{*}$ are independent for all $i$ and $t$ since normality is assumed. Denoting $\theta^{\prime}=\left[\beta^{\prime}, \rho, \lambda, \sigma^{2}\right]$ and $\eta^{\prime}=\left[\beta^{\prime}, \rho, \lambda\right]$, we can write the log-likelihood function as follows:

$$
\begin{aligned}
L(\theta)=-\frac{n(T-1)}{2} \ln 2 \pi-\frac{n(T-1)}{2} \ln \sigma^{2}+(T-1) \ln \left|\mathbf{S}_{n}(\rho)\right| \\
+(T-1) \ln \left|\mathbf{R}_{n}(\lambda)\right|-\frac{1}{2 \sigma^{2}} \sum_{t-1}^{T-1} \mathbf{V}_{n, t}^{*^{\prime}}(\eta) \mathbf{V}_{n, t}^{*}(\eta),
\end{aligned}
$$


with $\mathbf{V}_{n, t}^{*}=\mathbf{R}_{n}(\lambda)\left[\mathbf{S}_{n}(\rho) \mathbf{Y}_{n, t}^{*}-\mathbf{X}_{n, t}^{*} \beta\right]$. Moreover, Lee and $\mathrm{Yu}(2008,2009)$ show that under normality of the disturbances, this log-likelihood is the conditional loglikelihood of $\mathbf{Y}_{n, t}, t=1, \ldots, T$, conditional on $\overline{\mathbf{Y}}_{i, T}=\frac{1}{T} \sum_{t=1}^{T} \mathbf{Y}_{i, t}$, the time average for individual $i$.

Five different hypotheses are considered in this paper:

i) $H_{0}^{a}: \rho=\lambda=0$; This is the joint test. Under the alternative, at least one spatial parameter is different from 0 .

ii) $H_{0}^{b}: \rho=0$; This is a simple hypothesis test. $H_{a}: \rho \neq 0$. Under the alternative the specification is the SAR model.

iii) $H_{0}^{c}: \lambda=0$; This is the second simple hypothesis test. Under the alternative, the specification includes SAR errors.

iv) $H_{0}^{d}: \lambda=0$, with $\rho$ possibly different from $0 . H_{a}$ : the specification to be estimated is the general model (5).

v) $H_{0}^{e}: \rho=0$, with $\lambda$ possibly different from 0 . Under the alternative, the appropriate model is (5).

The joint test determines whether the spatial dimension matters. If it is not the case, there is no need to proceed further with the other tests. However, if the joint null hypothesis is rejected, the applied researcher should test the simple hypotheses to identify the most appropriate specification. If the two null hypotheses are both rejected, then one should test the last two null hypotheses to discriminate between the two alternative specifications of spatial autocorrelation. If both are again rejected than the appropriate specification is the $\operatorname{SARAR}(1,1)$ model.

Let us point out that the asymptotic distributions of our test statistics are not formally derived but they are likely to hold under assumptions developed in Lee and $\mathrm{Yu}$ (2008). ${ }^{7}$ In this paper, we focus instead on their finite sample performance by means of Monte Carlo simulations. The next subsections are devoted to the presentation of our LM and LR tests statistics. ${ }^{8}$

\subsection{Joint test statistics for $H_{0}^{a}: \rho=\lambda=0$}

Under the joint null hypothesis, the specification to be estimated is the usual fixed effects panel model. However, since the transformation used is different from the usual within transformation, we could wonder whether the OLS method is still appropriate. We have already shown that the variance-covariance matrix of the transformed error term is spherical. Moreover, the transformed regression (5) is linear since the $\mathbf{F}$ matrix is made of eigenvectors. Finally, the rank of $\mathbf{X}^{*}$ is not affected by the transformation. To prove the strict exogeneity assumption (needed for the consistency of OLS estimators), one uses the equality $\mathbf{F}_{T, T-1} \mathbf{F}_{T, T-1}^{\prime}=\mathbf{J}_{T}$, the original demeaning operator. Hence:

$$
\begin{aligned}
E\left[\mathbf{X}^{*^{\prime}} \mathbf{V}_{n, t}^{*}\right] & =E\left[\mathbf{X}^{\prime}\left(\mathbf{F}_{T, T-1} \otimes \mathbf{I}_{n}\right)\left(\mathbf{F}_{T, T-1}^{\prime} \otimes \mathbf{I}_{n}\right) \mathbf{V}_{n, t}\right] \\
& =E\left[\mathbf{X}^{\prime}\left(\mathbf{J}_{T} \otimes \mathbf{I}_{n}\right) \mathbf{V}_{\mathbf{n}, \mathbf{t}}\right]
\end{aligned}
$$

7. We computed qqplots for all our tests available from the authors upon request. They show that even in small samples, the tests already approach a $\chi^{2}$ distribution.

8. For the sake of brevity, we will only present final expressions: details are provided in the Appendix. 
Wooldridge (2002, p.268) has shown that (8), which corresponds to the usual within transformation, satisfies the strict exogeneity assumption. Since all assumptions for the OLS estimation to provide consistent estimators are met, the model under the null can be estimated by least squares. The joint LM statistic is then:

$$
L M_{J}=\tilde{Q}^{-1}\left[T_{22} \tilde{R}_{y}^{2}-2 T_{12} \tilde{R}_{v} \tilde{R}_{y}+\left(\tilde{D}+T_{11}\right) \tilde{R}_{v}^{2}\right]
$$

with, as notations,

$$
\begin{aligned}
\tilde{R}_{v} & =\frac{\sum_{t=1}^{T-1} \tilde{\mathbf{V}}_{n, t}^{{ }^{\prime}} \mathbf{M}_{n} \tilde{\mathbf{V}}_{n, t}^{*}}{\tilde{\sigma}^{2}} ; \\
\tilde{R}_{y} & =\frac{\sum_{t=1}^{T-1} \tilde{\mathbf{V}}_{n, t}^{*^{\prime}} \mathbf{W}_{n} \mathbf{Y}_{n, t}^{*}}{\tilde{\sigma}^{2}} ; \\
\tilde{D} & =\tilde{\sigma}^{-2} \sum_{t=1}^{T-1}\left(\mathbf{W}_{n} \mathbf{X}_{n, t}^{*} \tilde{\beta}\right)^{\prime} \mathbf{M}_{\mathbf{X}^{*}}\left(\mathbf{W}_{n} \mathbf{X}_{n, t}^{*} \tilde{\beta}\right) ; \\
\tilde{Q} & =\left(\tilde{D}+T_{11}\right) T_{22}-T_{12}^{2} .
\end{aligned}
$$

Also, $T_{11}=(T-1) \operatorname{tr}\left[\left(\mathbf{W}_{n}+\mathbf{W}_{n}^{\prime}\right) \mathbf{W}_{n}\right], T_{22}=(T-1) \operatorname{tr}\left[\left(\mathbf{M}_{n}+\mathbf{M}_{n}^{\prime}\right) \mathbf{M}_{n}\right], T_{12}=$ $(T-1) \operatorname{tr}\left(\left(\mathbf{M}_{n}^{\prime}+\mathbf{M}_{n}\right) \mathbf{W}_{n}\right)$ and $\mathbf{M}_{\mathbf{X}^{*}}$ is the usual annihilator matrix, namely $\mathbf{I}_{n(T-1)}-$ $\mathbf{X}^{*}\left(\mathbf{X}^{*^{\prime}} \mathbf{X}^{*}\right)^{-1} \mathbf{X}^{*^{\prime}}$. Finally, $\tilde{\mathbf{V}}_{n, t}^{*}=\mathbf{Y}_{n, t}^{*}-\mathbf{X}_{n, t}^{*} \tilde{\beta}$ are the residuals of the constrained model and $\tilde{\sigma}^{2}$ is the associated residual variance, simply estimated by OLS.

The LR test is based on the difference between the log-likelihood for unrestricted $\hat{\theta}$ and restricted models $\tilde{\theta}:{ }^{9}$

$$
L R=2[L(\hat{\theta})-L(\tilde{\theta})],
$$

with the coefficients evaluated at their unrestricted and restricted estimates. The LR test is asymptotically distributed as $\chi^{2}(q)$ with $q$ the number of constraints imposed. The unrestricted model is (5). If we concentrate out $\hat{\sigma}^{2}$ and $\hat{\beta}$, we get the following expression for the unrestricted log-likelihood function:

$$
\begin{aligned}
L(\hat{\theta})=-\frac{n(T-1)}{2}(1+\ln 2 \pi)-\frac{n(T-1)}{2} & \ln \frac{\sum_{t=1}^{T-1} \hat{\mathbf{V}}_{n, t}^{\prime *} \hat{\mathbf{V}}_{n, t}^{*}}{n(T-1)} \\
& +(T-1)\left[\ln \left|\mathbf{S}_{n}(\hat{\rho})\right|+\ln \left|\mathbf{R}_{n}(\hat{\lambda})\right|\right]
\end{aligned}
$$

with $\hat{\mathbf{V}}_{n, t}^{*}=\mathbf{R}_{n}(\hat{\lambda})\left[\mathbf{S}_{n}(\hat{\rho}) \mathbf{Y}_{n, t}^{*}-\mathbf{X}_{n, t}^{*} \hat{\beta}\right]$. Let us now consider the restricted model which is the classical linear model. Under the null hypothesis $H_{0}^{a}$ the restricted log-likelihood function is:

$$
L(\tilde{\theta})=-\frac{n(T-1)}{2} \ln 2 \pi-\frac{n(T-1)}{2} \ln \tilde{\sigma}^{2}-\frac{1}{2 \tilde{\sigma}^{2}} \sum_{t=1}^{T-1} \tilde{\mathbf{V}}_{n, t}^{* \prime} \tilde{\mathbf{V}}_{n, t}^{*}
$$

with $\tilde{\mathbf{V}}_{n, t}^{*}=\mathbf{Y}_{n, t}^{*}-\mathbf{X}_{n, t}^{*} \tilde{\beta}$. Again, concentrating out with respect to $\tilde{\sigma}^{2}$ and $\tilde{\beta}$, we obtain the following expression:

$$
L(\tilde{\theta})=-\frac{n(T-1)}{2}(1+\ln 2 \pi)-\frac{n(T-1)}{2} \ln \frac{\sum_{t=1}^{T-1} \tilde{\mathbf{V}}_{n, t}^{* \prime} \tilde{\mathbf{V}}_{n, t}^{*}}{n(T-1)}
$$

9. From now on coefficients topped by a denote estimators of the constrained models while those topped by a ^ denote estimators of the unconstrained models. 
The joint LR test statistic is therefore:

$$
L R_{J}=(n(T-1))\left[\ln \tilde{\sigma}^{2}-\ln \hat{\sigma}^{2}\right]+2(T-1)\left[\ln \left|\mathbf{S}_{n}(\hat{\rho})\right|+\ln \left|\mathbf{R}_{n}(\hat{\lambda})\right|\right],
$$

with $\tilde{\sigma}=\frac{1}{n(T-1)} \sum_{t=1}^{T-1} \tilde{\mathbf{V}}_{n, t}^{* \prime} \tilde{\mathbf{V}}_{n, t}^{*}$, the estimated residual variance of the constrained model and $\hat{\sigma}=\frac{1}{n(T-1)} \sum_{t=1}^{T-1} \hat{\mathbf{V}}_{n, t}^{* \prime} \hat{\mathbf{V}}_{n, t}^{*}$, the estimated residual variance of the unrestricted model.

This test indicates to the researcher whether the spatial dimension matters. When the null hypothesis is rejected, the econometric specification should explicitly account for spatial autocorrelation. As soon as one faces spatial data, this test should be performed since spatial autocorrelation causes traditional estimation methods to produce unreliable estimators. Let us however mention that when this statistic is significant, further tests should be conducted to find out the most appropriate specification. The aim of the four last tests is to fill this gap. The first two (ii and iii) are simple hypothesis tests. They thus test the presence of one type of spatial autocorrelation assuming the absence of the other. The last two (iv and v) are conditional test statistics. They are more general since they account for a possible misspecification of the model in terms of spatial autocorrelation.

\subsection{Simple hypothesis tests}

This section is concerned with the construction of marginal statistics to test for one particular form of spatial autocorrelation assuming the absence of the other.

\subsubsection{Marginal test statistics for $H_{0}^{b}: \rho=0$ (assuming $\lambda=0$ )}

The aim of these statistics is to test for spatial autocorrelation in the form of an endogenous spatial lag variable, i.e. a SAR model, assuming no SAR errors (i.e. $\lambda=0)$. The constrained model is the classical linear regression:

$$
\mathbf{Y}_{n, t}^{*}=\mathbf{X}_{n, t}^{*} \beta+\mathbf{V}_{n, t}^{*} \quad t=1, \ldots, T-1,
$$

with $\mathbf{V}_{n, t}^{*}$ distributed according to a normal distribution with zero mean and a variance-covariance matrix equal to $\sigma^{2} I_{n(T-1)}$. The unconstrained model is the SAR specification, formally written as:

$$
\mathbf{Y}_{n, t}^{*}=\rho \mathbf{W}_{n} y_{n, t}^{*}+\mathbf{X}_{n, t}^{*} \beta+\mathbf{V}_{n, t}^{*} \quad t=1, \ldots, T-1,
$$

and should be estimated by ML or GMM. Hence, the LM statistic is written as:

$$
L M_{\rho}=\frac{\sum_{t=1}^{T-1}\left(\tilde{\mathbf{V}}_{n, t}^{*^{\prime}} \mathbf{W}_{n} \mathbf{Y}_{n, t}^{*} / \tilde{\sigma}^{2}\right)^{2}}{\tilde{D}+T_{11}},
$$

with the same notations as before. As the constrained model can be estimated by least squares, $\tilde{\mathbf{V}}_{n, t}^{*}$ are the residuals of the OLS estimation of (15) and $\tilde{\sigma}^{2}$ is the estimate of the associated residual variance.

Using the same methodology as for the joint statistic, the LR counterpart is:

$$
L R_{\rho}=(n(T-1))\left[\ln \tilde{\sigma}^{2}-\ln \hat{\sigma}^{2}\right]+2(T-1)\left[\ln \left|\mathbf{S}_{n}(\hat{\rho})\right|\right],
$$

where $\tilde{\sigma}^{2}$ is the residual variance of the constrained model (15) whereas $\hat{\sigma}^{2}$ and $\hat{\rho}$ are estimated using the unconstrained model (16). 


\subsubsection{Marginal test statistics for $H_{0}^{c}: \lambda=0$ (assuming $\rho=0$ )}

Again for this test, the restricted model is (15). The specification under the alternative is:

$$
\begin{aligned}
& \mathbf{Y}_{n, t}^{*}=\mathbf{X}_{n, t}^{*} \beta+\mathbf{U}_{n, t}^{*} \\
& \mathbf{U}_{n, t}^{*}=\lambda \mathbf{M}_{n} \mathbf{U}_{n, t}^{*}+\mathbf{V}_{n, t}^{*} \quad t=1, \ldots, T-1 .
\end{aligned}
$$

The LM statistic is written as:

$$
L M_{\lambda}=\frac{\sum_{t=1}^{T-1}\left(\tilde{\mathbf{V}}_{n, t}^{*^{\prime}} \mathbf{M}_{n} \tilde{\mathbf{V}}_{n, t}^{*} / \tilde{\sigma}^{2}\right)^{2}}{T_{22}}
$$

Again, $\tilde{\mathbf{V}}_{n, t}^{*}$ are the residuals of the estimation of (15) and $\tilde{\sigma}^{2}$ is the estimate of the associated residual variance.

The corresponding LR statistic compares specification (19) with the model under the null, namely (15). The statistic is then:

$$
L R_{\lambda}=(n(T-1))\left[\ln \tilde{\sigma}^{2}-\ln \hat{\sigma}^{2}\right]+2(T-1) \ln \left|\mathbf{R}_{n}(\hat{\lambda})\right| .
$$

For these simple hypothesis statistics, the null is the absence of spatial autocorrelation. If statistics for a SAR model $\left(L M_{\rho}\right.$ and $\left.L R_{\rho}\right)$ are significant, spatial autocorrelation under the form of a endogenous spatial lag must be included in the regression. Similarly, if $L M_{\lambda}$ and $L R_{\lambda}$ are significant, the regression must account for spatially autocorrelated errors.

However, as shown in the Monte Carlo study, these statistics are affected by misspecification of the model in terms of spatial autocorrelation and tend to over reject the null. Their conditional version solve this drawback.

\subsection{Conditional hypothesis tests}

The first two statistics, $L M_{\lambda \mid \rho}$ and $L R_{\lambda \mid \rho}$, will test the presence of spatial error correlation when an endogenous spatial lag is already accounted for. The last two, $L M_{\rho \mid \lambda}$ and $L R_{\rho \mid \lambda}$, will detect a endogenous spatial lag when spatially autocorrelated errors are already included in the model.

\subsubsection{Condtional test statistics for $H_{0}^{d}: \lambda=0$ given $\rho \neq 0$}

The main difference with the simple hypothesis tests above is that the constrained model contains a spatial component implying the use of ML or GMM to get reliable estimators. The appropriate specification under the null is equation (16). When the null is rejected, the correct specification is the general model (5). Disturbances of the restricted model are:

$$
\mathbf{V}_{n, t}^{*}=\mathbf{S}_{n}(\rho) \mathbf{Y}_{n, t}^{*}-\mathbf{X}_{n, t}^{*} \beta \quad t=1, \ldots, T-1,
$$

which can be replaced by the ML residuals of (16) when implementing the LM test.

The conditional test for spatially autocorrelated errors in the presence of an endogenous spatial lag is written as:

$$
L M_{\lambda \mid \rho}=\frac{\left(\sum_{t=1}^{T-1} \tilde{\mathbf{V}}_{n, t}^{*^{\prime}} M_{n} \tilde{\mathbf{V}}_{n, t}^{*} / \tilde{\sigma}^{2}\right)^{2}}{T_{22}-\left(\tilde{T}_{\lambda \rho}\right)^{2} * \operatorname{var}(\tilde{\rho})}
$$


with $\operatorname{var}(\tilde{\rho})$, the variance of the autoregressive coefficient estimated under the constrained model and $\tilde{T}_{\lambda \rho}=(T-1) \operatorname{tr}\left[\mathbf{M}_{n}^{\prime} \mathbf{W}_{n} \mathbf{S}_{n}(\tilde{\rho})^{-1}+\mathbf{M}_{n} \mathbf{W}_{n} \mathbf{S}_{n}(\tilde{\rho})^{-1}\right] . \tilde{\mathbf{V}}_{n, t}^{*}$ are the residuals of the constrained model (16).

To the difference of simple hypotheses tests, the LR statistic will include an additional term, the jacobian of the transformation contained in the log-likelihood function of the constrained model.

$$
\begin{aligned}
L R_{\lambda \mid \rho}=(n(T-1))\left[\ln \tilde{\sigma}^{2}-\right. & \left.\ln \hat{\sigma}^{2}\right] \\
& +2(T-1)\left[\ln \left|\mathbf{S}_{n}(\hat{\rho})\right|+\ln \left|\mathbf{R}_{n}(\hat{\lambda})\right|-\ln \left|\mathbf{S}_{n}(\tilde{\rho})\right|\right]
\end{aligned}
$$

with $\tilde{\sigma}^{2}$ and $\tilde{\rho}$, the residual variance and the estimated spatial autoregressive parameter of (16). Moreover, $\hat{\sigma}^{2}, \hat{\rho}$ and $\hat{\lambda}$ are respectively the residual variance, spatial parameter for the endogenous spatial lag and the spatial parameter for the spatially autocorrelated error component, estimated with the unconstrained model (5).

\subsubsection{Conditional tests statistics for $H_{0}^{e}: \rho=0$ given $\lambda \neq 0$}

The unconstrained model is the general one (5) while the constrained model includes SAR errors, i.e. equation (19). Again, the latter should be estimated by ML. Its error term can be written as:

$$
\mathbf{V}_{n, t}^{*}=\mathbf{R}_{n}(\lambda)\left[\mathbf{Y}_{\mathbf{n}, \mathbf{t}}{ }^{*}-\mathbf{X}_{n, t}^{*} \beta\right] \quad t=1, \ldots, T-1 .
$$

The conditional LM statistic is:

$$
L M_{\rho \mid \lambda}=\frac{\sum_{t=1}^{T-1}\left(\tilde{\mathbf{V}}_{n, t}^{*^{\prime}} \mathbf{R}_{n}(\tilde{\lambda}) \mathbf{W}_{n} \mathbf{Y}_{n, t}^{*} / \tilde{\sigma}^{2}\right)^{2}}{\tilde{I}_{11}-\tilde{I}_{12} \tilde{I}^{22} \tilde{I}_{21}} .
$$

$\tilde{I}^{22}$ is the variance-covariance matrix of the non-constrained parameters, namely $\tilde{\lambda}, \tilde{\beta}, \tilde{\sigma}^{2}$. Moreover, the other terms are defined as follows:

$$
\begin{gathered}
\tilde{I}_{11}=(T-1) \operatorname{tr}(\mathbf{W})^{2}+\frac{1}{\tilde{\sigma}^{2}} \sum_{t=1}^{T-1}\left[\left(\mathbf{R}_{n}(\tilde{\lambda}) \mathbf{W}_{n} \tilde{\mathbf{X}}_{n, t}^{*} \tilde{\beta}\right)^{\prime}\left(\mathbf{R}_{n}(\tilde{\lambda}) \mathbf{W}_{n} \mathbf{X}_{n, t}^{*} \tilde{\beta}\right)\right] \\
+(T-1) \operatorname{tr}\left[\left(\mathbf{R}_{n}(\tilde{\lambda}) \mathbf{W}_{n} \mathbf{R}_{n}(\tilde{\lambda})^{-1}\right)^{\prime}\left(\mathbf{R}_{n}(\tilde{\lambda}) \mathbf{W}_{n} \mathbf{R}_{n}(\tilde{\lambda})^{-1}\right)\right] \\
\tilde{I}_{12}^{\prime}=\left[\begin{array}{c}
\frac{1}{\tilde{\sigma}^{2}} \sum_{t=1}^{T-1} \mathbf{X}_{n, t}^{*^{\prime}} \mathbf{R}_{n}(\tilde{\lambda})^{\prime} \mathbf{R}_{n}(\tilde{\lambda}) \mathbf{W}_{n} \mathbf{X}_{n, t}^{*} \tilde{\beta} \\
(T-1) \operatorname{tr}\left[\left(\mathbf{M}_{n} \mathbf{R}_{n}(\tilde{\lambda})^{-1}\right)^{\prime} \mathbf{R}_{n}(\tilde{\lambda}) \mathbf{W}_{n} \mathbf{R}_{n}(\tilde{\lambda})^{-1}+\mathbf{M}_{n} \mathbf{W}_{n} \mathbf{R}_{n}\left(\tilde{\lambda}^{-1}\right)\right] \\
0
\end{array}\right] .
\end{gathered}
$$

All parameters involved in this test come from the constrained model, which is estimated by ML. If the statistic is significant, the most appropriate specification is the general model (5) since both spatial autocorrelation types are present.

To test this null hypothesis with a LR test, one has to apply the following formula:

$$
\begin{aligned}
L R_{\rho \mid \lambda}=(n(T-1))\left[\ln \tilde{\sigma}^{2}-\right. & \left.\ln \hat{\sigma}^{2}\right] \\
& +2(T-1)\left[\ln \left|\mathbf{S}_{n}(\hat{\rho})\right|+\ln \left|\mathbf{R}_{n}(\hat{\lambda})\right|-\ln \left|\mathbf{R}_{n}(\tilde{\lambda})\right|\right] .
\end{aligned}
$$




\section{Monte Carlo simulations}

To assess the finite-sample properties of our tests, we conduct some Monte Carlo experiments. Two different Data Generating Processes (DGP) are suggested. The first implements the ideal framework for the statistics and will be referred to the Ideal DGP (IDGP). The second DGP assesses the robustness of the proposed statistics. It is based on the format used in earlier studies in the spatial econometric literature (Anselin and Florax, 1995; Baltagi et al., 2003, 2007) and panel data models (Nerlove, 1971) and is viewed as the Robust DGP (RDGP).

The IDGP is set as follows:

$$
\begin{aligned}
y_{i, t} & =\rho \mathbf{W}_{\mathbf{i} . \mathbf{y}_{t}}+\beta x_{i, t}+u_{i, t} \\
u_{i, t} & =\lambda \mathbf{M}_{\mathbf{i} .} \mathbf{u}_{t}+v_{i, t},
\end{aligned}
$$

where $\mathbf{W}_{\mathbf{i}}$. is the $i^{\text {th }}$ row of the spatial weight matrix associated to the endogenous spatial lag while $\mathbf{M}_{\mathbf{i}}$. is the $i^{\text {th }}$ row of the spatial weight matrix associated with the spatially autocorrelated errors. Also, $x_{i, t} \sim N\left(\mu_{i}, 1\right)$ with $\mu \sim U(0,1) * 10$ and $v_{i, t} \sim I N(0,1)$. The $x$ variable is thus defined with a different mean for each individual. We decided not to include a constant for the sake of simplicity.

Two different patterns for interaction schemes are used in our simulations: $\mathbf{W} \neq \mathbf{M}$ and $\mathbf{W}=\mathbf{M}$. In the former, we set $\beta=1$ since this value is commonly used in Monte Carlo studies. For the latter however, to avoid problems of identification of $\lambda$ and $\rho, \beta x_{i, t}$ should contribute to the explanation of $y_{i, t}$ (i.e $\beta \neq 0$ ). According to LeSage and Pace (2009), the $\beta$ value should be set relatively higher than the residual variance to avoid low signal-to-noise problems. We thus set $\beta=3$ for that case. Hence, we consider $\beta=1$ for $\mathbf{W} \neq \mathbf{M}$ and $\beta=3$ when $\mathbf{W}=\mathbf{M}$.

The Robust DGP has the following expression:

$$
\begin{aligned}
y_{i, t} & =\rho \mathbf{W}_{\mathbf{i} . \mathbf{y}_{t}+\beta x_{i, t}+\mu_{i}+u_{i, t}} \\
x_{i, t} & =0.1 t+0.5 x_{i, t-1}+z_{i, t} \\
u_{i, t} & =\lambda \mathbf{M}_{\mathbf{i} .} \mathbf{u}_{t}+v_{i, t},
\end{aligned}
$$

with the same notations as above. To operationalize this process, parameters values and random variables are set as follows:

$$
\begin{aligned}
\beta & =1 ; 3, \\
\mu_{i} & \sim U(-5,5), \\
v_{i, t} & \sim I N(0,1), \\
z_{i, t} & \sim U(-0.5,0.5) .
\end{aligned}
$$

Initial individual values are defined as $x_{i, 0}=5+10 z_{i, 0}$. Again, two values were assigned to $\beta$ for the same reason as above. The main differences between the two DGP come from the serial autocorrelation of the explanatory variable and the inclusion of a trend. Hence, the RDGP will examine whether our tests are affected by the presence of non-modeled serial autocorrelation. $\mathbf{W}$ is a Rook-type of order 1 weight matrix while $\mathbf{M}$ is a Rook-type of either order 1 or 2 weight matrix. ${ }^{10}$ Both $\mathbf{W}$ and

10. The order of contiguity represents the closeness of one region with its neighbours. Order 1 means that the neighbourhood is constituted by adjacent regions. Order 2 neighbourhood is made of the neighbours of neighbours, etc. 
$\mathbf{M}$ are row-normalized.

We selected several panel sizes $(N=25,49,81)$ all defined on a regular grid for different time periods $(T=7,10)$. In these simulations, we let both spatial parameters $(\rho$ and $\lambda)$ vary over the set $[0,0.9]$ by increment of 0.1 . Experiments were replicated 1000 times. For the sake of compactness, we will only present two sample sizes: $N=81, T=7$ and $N=49, T=10{ }^{11}$ All computations were performed in MATLAB.

The results are summarized in Tables 1 to 11. Each Table presents the power of one type of statistic for one DGP (except otherwise explicitly mentioned). Under the null, the power of a test equals its size, which we set to 0.05. For the sake of clarity, all sizes are highlighted in bold. The two different interaction schemes are set as follows. Either both spatial weight matrices are of the Rook type of order 1 or $\mathbf{W}$ is of the Rook-type of order 1 while $\mathbf{M}$ is of Rook-type of order 2 .

\section{Insert Tables 1 to 6 around here}

Let us first study the performance of the joint statistic in the IDGP, summarized in Table 1. Under the null, the sample does not contain any spatial autocorrelation. Sizes of $L M_{J}$ and $L R_{J}$ are close to the $5 \%$ value. For example, when $\mathbf{W} \neq \mathbf{M}$, $N=81$ and $T=7$, sizes of $L M_{J}$ and $L R_{J}$ are respectively of 0.04 and 0.043 . Furthermore, the tests perform very well since a $100 \%$ rejection rate is obtained as soon as $\rho=0.2$ and $\lambda=0.3 .{ }^{12}$ We finally note that results for the Robust DGP (Table 2) are very similar, implying that serial correlation does not seem to affect the joint statistics.

Tables 3 and 4 report the behaviour of simple hypothesis tests for the presence of spatially autocorrelated errors $\left(L M_{\lambda}\right.$ and $\left.L R_{\lambda}\right)$. As these test statistics perform similarly in both DGP, we will analyze results for the Ideal one (Table 3 ). When the specification does not suffer from misspecification in terms of spatial autocorrelation (i.e $\rho=0$ ), the size of $L M_{\lambda}$ is close to its theoretical value in all situations considered (2 spatial schemes and 2 sample sizes). For example, when $\mathbf{W}=\mathbf{M}, N=49$ and $T=$ 10, the size of $L M_{\lambda}=0.055$. We also note that the equivalent $L R$ statistic is more conservative. Moreover, both tests have good power since we observe full rejection rate (of the null) as soon as $\lambda=0.5$ and powers increase smoothly. In case of model misspecification, empirical sizes are very misleading. Indeed, considering $\rho=0.5$ in the case $\mathbf{W} \neq \mathbf{M}, N=49, T=10$, the sizes of $L M_{\lambda}$ and $L R_{\lambda}$ are respectively of 0.978 and 0.949 against the theoretically 0.05 . The explanation lies in the construction of the simple hypothesis tests. As constrained models for both tests are identical, simple hypothesis statistics do not discriminate between spatially autocorrelated errors and endogenous spatial lag. Note that the sizes are even worse when the spatial weight matrices are identical.

The outcomes of the two simple hypothesis tests for the presence of an endogenous spatial lag $\left(L M_{\rho}\right.$ and $\left.L R_{\rho}\right)$ are presented in Tables 5 and 6 . We first note that size of $L M_{\rho}$ is close to the theoretical value, no matter the scenario. Also, as for $L R_{\lambda}$, $L R_{\rho}$ is undersized. The power of the two statistics are very good since we obtain

11. The results for the other designs are very similar. We also performed Monte Carlo experiments for negative values of spatial parameters and got very similar results.

12. When the figure is not reported in the table, this means that the rejection rate was $100 \%$. This convention has been adopted for all following tables. 
a full rejection rate as soon as $\rho=0.4$. We finally observe that these two tests are also affected by the presence of spatially autocorrelated errors and specifically when both spatial weight matrices are identical Again, all the results mentioned are valid for the Robust DGP.

\section{Insert Tables 7 to 11 around here}

The effect of model misspecification on simple hypothesis tests leads us to the analysis of conditional statistics where it is explicitly accounted for. The performance of the last tests are illustrated in Tables 7 to 11 . We first focus on the tests of the presence of spatially autocorrelated errors when an endogenous spatial is already accounted for in the specification (i.e $L M_{\lambda \mid \rho}$ and $L R_{\lambda \mid \rho}$ ). Their outcomes are summarized in Tables 7 and 8. We will first study the performance of these two tests in the Ideal DGP (without any serial correlation). As for simple hypothesis tests, sizes are reported in the two first columns of the Tables, in bold. The empirical size of the $L M$ statistic corresponds to its theoretical counterpart, no matter the importance of the endogenous spatial lag. For example, in the $\mathbf{W}=\mathbf{M}, \mathbf{N}=49, \mathrm{~T}=10$ case, when $\rho=0.8$, the size of $L M_{\lambda \mid \rho}=0.045$. We also note that $L R_{\lambda \mid \rho}$ is undersized. The power of these two statistic is quite high since we observe full rejection of the null hypothesis as soon as $\lambda=0.5$. Let us remark that the power of these statistics are not affected either by the value of the free spatial parameter $(\rho)$ or the possible equality of the two spatial weight matrices. Finally, Table 8 which presents results for the Robust DGP, exhibits similar outcomes.

The last experiments focus on the test statistics to detect an endogenous spatial lag when spatially autocorrelated errors are already present in the specification $\left(L M_{\rho \mid \lambda}\right.$ and $\left.L R_{\rho \mid \lambda}\right)$. The results are reported in Tables 9 to 11 . In the Ideal DGP (Table 9), these statistics behave the same way as the two previous one. Empirical size of the $L M$ statistic is similar to the required $5 \%$ while the $L R$ is undersized. Moreover, the value of $\lambda$ (the unconstrained parameter) does not affect the properties of both tests. Looking at power, as soon as $\rho$, the constrained parameter, is set to 0.3 , we observe a full rejection rate of the null hypothesis. Let us finally note that in this DGP, both $L M$ and $L R$ statistics are not affected by the possible equality of spatial weight matrices. Results for the Robust DGP, summarized in Tables 10 and 11, do not show any significant difference with the Ideal case.

\section{The Feldstein-Horioka puzzle revisited}

In their seminal article, Feldstein and Horioka (1980) (FH) found a high correlation between domestic saving and domestic investment rates for OECD countries in a cross-section setting. They interpret these findings as evidence against capital mobility between countries. Indeed, if capital was perfectly mobile, domestic saving would seek out the highest returns, no matter the location. This implies that an exogenous shock in investment would be financed by a perfectly elastic supply of global capital. On the contrary, if capital is immobile, we would observe a one-to-one relationship between domestic investment and saving rates since the latter should be invested locally. The FH result thus poses an uncomfortable puzzle since the conventional view in most exchange rate and open-economy macroeconomic models highlights the importance of capital mobility.

The literature challenged the FH result in several ways but obtained the same high correlation result. This persistence gave birth to the FH paradox. Authors first 
tested the FH hypothesis (high correlation) on different databases without being able to reject the FH findings (Feldstein, 1983; Penati and Dooley, 1984; Dooley et al, 1987; Tesar, 1991).

The application of panel data model to the $\mathrm{FH}$ puzzle was initiated by Krol (1996). He argued that time-averaged data (cross-section) bias results against capital mobility since they obscure variation in current account and thus, discrepancies between investment and saving (an argument already pointed out by Sinn, 1992). He partly solved the paradox but Jansen (2000) challenged his findings invoking the presence of Luxembourg in his sample. Krol's study was followed by many others applying either classical panel data models or more sophisticated methods as panel smoothing (Fouquau et al., 2008) or cointegrated panel data models (Coiteux and Olivier, 2000) without being able to solve the puzzle.

All the works mentioned above make the implicit assumption that countries are independent from each others. More precisely, investment rates (the dependent variable in $\mathrm{FH}$ ) are assumed to be independent across countries. We propose here to test this hypothesis, which is crucial since its violation causes classical estimators to be unreliable. Hence, we test the presence of spatial autocorrelation in the investmentsavings relationship in a fixed effects panel data model. The choice of the fixed effects panel framework comes from the literature. All panel data model estimations conclude to the presence of individual fixed effects and the absence of temporal effects (see for instance Jansen, 2000; Corbin, 2001). We show that spatial autocorrelation is indeed present and ignoring it leads to an upward biased estimator, which favors capital immobility.

In this paper, we consider investment and saving rates for 24 OECD countries between 1960 and 2000 (41 periods). ${ }^{13}$ Ratios of investment come from the Penn World Table and are defined as investment share of real gross domestic product per capita. Ratio of savings are defined as the percentage change of current savings to GDP. ${ }^{14}$ Table 12 reports some descriptive statistics for the two variables under consideration.

We consider first the results of the usual fixed effects panel data model applied to this dataset. We split the sample into three sub-periods. The first one covers the eleven first periods $(1960-1970)$. The reason is that in the late 60 's, a trend of liberalizing capital flows and deregulating financial markets occurred in most of the countries belonging to OECD (OECD, 1990). Then, we divide the remaining observations into two equal sub-periods to allow comparison with the first period.

We first estimate a traditional fixed effects panel model of the investment-saving correlation for the three sub-periods:

$$
\mathbf{I}_{t}=\mu+\beta \mathbf{S}_{t}+\epsilon_{t}, \quad t=1, \ldots, T,
$$

where, for each time period $t, \mathbf{I}_{t}$ is the $n \times 1$ vector of investment rates for all countries, $\mathbf{S}_{t}$ is the $n \times 1$ vector of saving rates, $\mu$ is the $n \times 1$ vector of individual fixed effects, and $\epsilon_{t}$ is a $n \times 1$ vector of assumed well behaved disturbances. Table 13 reports the results.

We clearly see a decrease in the relationship between domestic saving and investment rates across time since we start from a value of 0.932 for the sixties and end

13. Countries considered are: Australia, Austria, Belgium, Canada, Switzerland, Denmark, Spain, Finland, France, United Kingdom, Greece, Ireland, Iceland, Italy, Japan, Mexico, Netherlands, Norway, New Zealand, Portugal, Republic of Korea, Sweden, Turkey and United States.

14. They are actually computed as subtracting consumption share and government share of real gross domestic product per capita, from 100. 
up with a value of 0.442 for the last sub-period. This indicates an upsurge in capital mobility, as noted by OECD (1990).

However, these results are obtained under the restrictive and unlikely assumption of no spatial autocorrelation between countries, which are considered as "independent islands". As mentioned above, capturing spatial interactions requires the setup of an exogenous spatial weight matrix. Let us underline that, contrary to the time series case, there is no unique natural ordering of cross section observations in spatial econometrics. The interaction matrix $\mathbf{W}$ is then the fundamental tool to define a "relevant" order structure by specifying a "neighbourhood set" for each observation. More precisely, each observation is connected to a set of "neighbouring" observations by means of an exogenous pattern introduced in the interaction matrix. By convention an observation is not a neighbour to itself so that elements on the main diagonal of $\mathbf{W}$ are set to zero $\left(w_{i i}=0\right)$, whereas in each row $i$, a non zero element $w_{i j}$ defines $j$ as being a neighbor of $i$ and further specifies the way $i$ is connected to $j$.

Many different spatial weights matrices may then be specified to study the same issue and it may be difficult to identify the most "relevant" matrix, leaving the room for some arbitrariness. Sensitivity analysis of the results plays then an important role in practice. Traditionally, connectivity has been understood as geographical proximity, various weights matrices based on geographical space have therefore been used in the spatial econometric literature such as contiguity, nearest neighbors and geographical distance based matrices. However the definition is in fact much broader and can be generalized to any network structure to reflect any kind of interactions between observations. As also underlined by Durlauf et al. (2005, p. 643-645), what really matters when adapting these methods to growth econometrics is the identification of the appropriate notion of space and of the appropriate similarity or interaction measure. By analogy to Akerlof (1997) countries may be considered as localized in some general socio-economic and institutional or political space defined by a range of factors. Implementation of spatial methods requires then to identify accurately their location in such a general space. Ideally, such a matrix should be theory based but this is beyond the scope of the present paper.

We adopt here a heuristic approach by specifying two different interaction matrices, frequently used in the literature, to test the robustness of our results with regard to the choice of the spatial weight matrix. The first interaction matrix we use is based on inverse distance. The general term of this matrix is defined as $w_{i j}=d_{i j}^{-1}$ if $i \neq j$, where $d_{i j}$ is the arc-distance between capitals of countries $i$ and $j$. The second interaction matrix is the binary seven nearest neighbours weight matrix whose general term is defined as follow:

$$
\begin{cases}w_{i j}=1 & \text { if } d_{i j} \leq d_{i}, i \neq j \\ w_{i j}=0 & \text { if } d_{i j}>d_{i}, i \neq j,\end{cases}
$$

where $d_{i}$ is the $7^{\text {th }}$ order smallest arc-distance between countries $i$ and $j$ such that each country $i$ has exactly 7 neighbours. Note that the second matrix is sparse whereas the preceding one is not, reflecting quite different interaction patterns. Both interaction matrices are row-normalized as usually done in the applied literature. Finally, let us point out that we assume the same spatial scheme for both the spatially autocorrelated errors and the endogenous spatial lag.

Table 14 summarizes the results of the application of the spatial autocorrelation tests developed above. We first observe similar results for the two weight matrices, which pleads for the robustness against the choice of the interaction scheme. We 
will thus focus on the first spatial scheme. In the first sub-period, which covers the sixties, the joint tests $\left(L M_{J}\right.$ and $\left.L R_{J}\right)$ are not significant. Hence, spatial autocorrelation is not an issue and investment rates can be viewed as independent between countries. Given this result, the other tests should not be computed and the model can be estimated with traditional fixed effects methods. Moving to the second subperiod, we observe significant joint tests. Spatial autocorrelation should thus be accounted for in the econometric regression. Looking at simple hypothesis tests, we note that the two types of spatial autocorrelation are also significant. However, at this stage, we cannot determine what type of spatial autocorrelation characterizes the sample. We thus study their conditional counterparts to collect further information: $L M_{\rho \mid \lambda}$ and $L R_{\rho \mid \lambda}$ are significant. Accounting for spatially autocorrelated errors in the specification does not solve the problem since an endogenous spatial lag is still present. However, the two other conditional statistics $\left(L M_{\lambda \mid \rho}\right.$ and $\left.L R_{\lambda \mid \rho}\right)$ are not significant. Thus, once an endogenous spatial lag is included in the econometric specification, no residual spatial autocorrelation remains. For this sub-period, the adequate econometric specification consists in adding an extra regressor, the spatially lagged investment rate, to equation (31), which yields a SAR model.

The analysis of the last sub-period provides the same conclusion. Joint statistics are significant, marginal tests all reject their respective null hypothesis, and the study of conditional statistics reveals that an econometric specification containing an endogenous spatial lag is the most adequate to capture spatial autocorrelation. Note that $L R$ statistics are more conservative than their $L M$ counterpart. Table 14 finally reports a test for the presence of individual effects. For the first subperiod, we apply the traditional Chow test since spatial autocorrelation is not an issue. However, for the last two subperiods, we use a LR test to assess the significant of individual effects since the Chow statistic is affected by the presence of spatial autocorrelation (Anselin, 1988). Results indicate that individual effects should be accounted for in an econometric specification.

Formally, the SAR specification is written as follows:

$$
\mathbf{I}_{t}=\mu+\rho \mathbf{W I}_{t}+\beta \mathbf{S}_{t}+\epsilon_{t}, \quad t=1, \ldots, T .
$$

In this model the investment rate of a given country $i$ depends on investment rates in "neighboring" countries in addition to its own saving rate. It is estimated in implicit form with the maximum likelihood estimation method proposed in Lee and $\mathrm{Yu}$ (2008). However, we need to express its reduced form to obtain estimates of the spillovers effects as follows:

$$
\mathbf{I}_{t}=\left(\mathbf{I}_{\mathbf{n}}-\rho \mathbf{W}\right)^{-1} \mu+\left(\mathbf{I}_{\mathbf{n}}-\rho \mathbf{W}\right)^{-1} \beta \mathbf{S}_{t}+\left(\mathbf{I}_{\mathbf{n}}-\rho \mathbf{W}\right)^{-1} \epsilon_{t},
$$

This model implies that the saving rate of one country spills over countries. It is then possible to quantify the effect of a change in the saving rate of a given country $i$ on its own investment rate but also on the investment rate of all other countries in the sample. We therefore obtain the matrix of the derivatives of $\mathbf{I}_{t}$ relative to $\mathbf{S}_{t}$ :

$$
\boldsymbol{\Xi}_{I}^{S} \equiv \frac{\partial \mathbf{I}_{t}}{\partial \mathbf{S}_{t}}=\beta(\mathbf{I}-\rho \mathbf{W})^{-1}
$$

The diagonal elements of this matrix contain the direct effects including own spillover effects, which are inherently heterogeneous in presence of spatial autocorrelation, 
whereas off-diagonal elements represent indirect effects. Using obvious notations, we have:

$$
\frac{\partial \mathbf{I}_{t, i}}{\partial \mathbf{S}_{t, i}} \equiv\left(\boldsymbol{\Xi}_{\mathbf{I}}^{\mathbf{S}}\right)_{t, i i} \quad \text { and } \quad \frac{\partial \mathbf{I}_{t, i}}{\partial \mathbf{S}_{t, j}} \equiv\left(\boldsymbol{\Xi}_{\mathbf{I}}^{\mathbf{S}}\right)_{t, i j}
$$

Note that the own derivative for country $i$ includes the feedback effect where country $i$ affects country $j$ and country $j$ also affects country $i$ as well as longer paths which might go from country $i$ to $j$ to $k$ and back to $i$. The magnitude of those effects will depend on: (1) the degree of interaction among countries which is governed by the W matrix used in the model, (2) the parameter $\rho$ measuring the strength of spatial dependance between countries and (3) the parameter $\beta$.

Moreover, the sum across the $i^{\text {th }}$ row represents the total impact on the investment rate of country $i$ of a change of the saving rates by the same amount across all the $n$ countries of the sample. The sum down the $j^{\text {th }}$ column yields the total impact over the investment rates over all the $n$ countries of the sample of a change of the saving rate in country $j$, which is of particular interest here. The average direct impact is therefore defined as $n^{-1} \operatorname{tr}\left(\widehat{\boldsymbol{\Xi}_{\mathbf{I}}} \mathbf{S}\right)$ whereas the average total impact is defined as $n^{-1} \iota \widehat{\Xi}_{\mathbf{I}}^{\mathbf{S}} \iota$ where $\iota$ is the $n \times 1$ sum vector. Note that in our SAR model with a row-standardized interaction matrix the average total impact further simplifies to $(1-\rho)^{-1} \beta$. Finally the average indirect impact is by definition the difference between the average total impact and the average direct impact. ${ }^{15}$

Table 15 provides estimation results of equation (33) using both interaction matrices for the last two subperiods along with the within estimation of equation (31) for the first subperiod. The results confirm the view expressed in OECD (1990) that capital mobility, and thus interactions between countries, increased from the late sixties on. We note that the coefficients of the saving rates are significant as well as the spatial autocorrelation coefficients whatever the interaction matrix used, highlighting the robustness of our estimates with regard to the choice of the interaction scheme. The significance of the spatial autocorrelation parameter is crucial: first it implies that the investment rate in one country cannot be considered as independent from those of other countries and second it implies that the saving-retention coefficient is characterized by interactive heterogeneity.

The estimated matrix (35) is therefore presented in Table 16 for the second subperiod and in Table 17 for the last subperiod using the inverse distance matrix. ${ }^{16}$ We represent in bold the estimated direct effect including the own spillover effect (main diagonal). Note that all impact coefficients are significant using the Delta method. The average direct impact is 0.632 for the second subperiod and 0.367 for the last subperiod. Comparing these results with Table 13, the usual within estimators appear to be upward biased. Accounting for spatial autocorrelation thus pleads for capital mobility. Indeed, for the second sub-period, we see a drop of the saving-retention coefficient from 0.707 to 0.632 . For the last sub-period, the coefficient drops from 0.442 to 0.367 . The average indirect impact is 0.613 for the second subperiod and 0.396 for the last subperiod.

Let us finally remark that the tests indicate the predominance of an endogenous spatial lag to capture spatial autocorrelation. Spatial autocorrelation can thus be

15. LeSage and Pace (2009, chap. 2) present a comprehensive analysis of those effects along with some useful summary measures in the cross-section setting. Their extension to our panel data setting is straightforward. See also Kelejian et al. $(2006,2008)$ for some applications.

16. Results obtained using the 7 nearest-neighbours matrix are similar and available upon request from the authors. 
viewed as a substantive process and should deserve attention in the economic modeling of the investment-saving correlation. Until now, this has been overlooked in the literature.

\section{Conclusion}

This paper has developed several LM and LR statistics to test for the presence and the type of spatial autocorrelation in a fixed effects panel data model. Our estimation procedure is based on the Lee and $\mathrm{Yu}$ (2008) paper, which allows the estimation of a fixed effects spatial panel data by Maximum Likelihood. We suggest a general joint statistic as a preliminary test to detect spatial autocorrelation. This test is shown to be powerful against both types of spatial autocorrelation. If the joint statistic is significant, the two simple hypothesis tests should be applied. Each one tests for the presence of one type of spatial autocorrelation: spatially autocorrelated errors or endogenous spatial lag. When only one test is significant, the final specification should be set accordingly.

However, Monte Carlo results show that simple hypothesis tests are affected by model misspecification in terms of spatial autocorrelation. Simple hypothesis tests will thus generally be both significant, which does not allow to determine the best way to model spatial autocorrelation. To solve this drawback, conditional statistics are derived. If only one of them is significant, the best specification can be determined. For example, if only $L M_{\rho \mid \lambda}$ and $L R_{\rho \mid \lambda}$ are significant, the best spatial specification will include an endogenous spatial lag. If however, the two remain significant, the econometric regression should include both types of spatial autocorrelation. Furthermore, Monte Carlo experiments showed that the performance of the statistics are very good and unaffected by the DGP chosen.

Finally, the application of the tests to the Feldstein-Horioka puzzle shows that spatial autocorrelation is an important issue and should be accounted for in the econometric specification of the panel data model under the form of an endogenous spatial lag. Otherwise, results would be biased. We observed that explicitly accounting for spatial autocorrelation in a simple model leads to a drop of the investmentsaving relationship suggesting more capital mobility. The next step would be to theoretically derive an investment-saving relationship that accounts for spatial autocorrelation with a special focus on the relevant interaction matrix to adopt. 


\section{References}

Anselin L. 1988a. Spatial Econometrics, Methods and Models. Kluwer Academic Publishers: Dordrecht.

Anselin L. 1988b. Lagrange Multiplier Tests Diagnostics for Spatial Dependence and Spatial Heterogeneity. Geographical Analysis 20: 1-17.

Anselin L. 2001. Rao's Score Test in Spatial Econometrics. Journal of Statistical Planning and Inference 97: 113-139.

Anselin L. 2003. Spatial Externalities. Spatial Multipliers and Spatial Econometrics. International Regional Science Review 26: 153-166.

Anselin L. 2006. Spatial Econometrics. In Palgrave Handbook of Econometrics: Volume 1, Econometric Theory, Mills TC, Patterson K (eds). Palgrave Macmillan: Basingstoke.

Anselin L, Bera AK, Florax R, Yoon MJ. 1996. Simple diagnostic tests for spatial dependence. Regional Science and Urban Economics 26:1, 77-104.

Anselin L, Bera AK. 1998. Spatial dependence in linear regression models with an introduction to spatial econometrics. In Handbook of Applied Economic Statistic, Ullah A, Giles D (eds). Marcel Dekker: New York.

Anselin L, Florax R. 1995, Small sample properties of tests for spatial dependence in regression models: some further results. In New Directions in Spatial Econometrics, Anselin L, Florax R (eds). Springer: Berlin

Anselin L, Bongiovanni RG, Lowenberg-DeBoer J. 2004. A Spatial Econometric Approach to the Economics of Site-Specific Nitrogen Management in Corn Production. American Journal of Agricultural Economics 86: 675-687.

Anselin L, Le Gallo J, Jayet H. 2008. Spatial Panel Econometrics. In The Econometrics of Panel Data, Fundamentals and Recent Developments in Theory and Practice (3rd Edition), Matyas L, Sevestre P (eds). Kluwer Academic Publishers: Dordrecht.

Baltagi BH. 2008, Econometric Analysis of Panel Data, Fourth Edition. John Wiley and Sons: Chichester.

Baltagi BH, Song SH, Koh W. 2003. Testing Panel Data Regression Models with Spatial Error Correlation. Journal of Econometrics 117: 123-150.

Baltagi BH, Egger P, Pfaffermayr M. 2007a. A Generalized Spatial Panel Data Model with Random Effects. Working paper. Syracuse University.

Baltagi BH, Egger P, Pfaffermayr M. 2007b. Estimating models of complex FDI: Are there third-country effects? Journal of Econometrics 140: 260-281.

Baltagi BH, Egger P, Pfaffermayr M. 2008. Estimating regional trade agreement effects on FDI in an interdependent world. Journal of Econometrics 145: 194208. 
Baltagi BH, Song SH, Jung BC, Koh W. 2007. Testing for Serial Correlation, Spatial Autocorrelation and Random Effects using Panel Data. Journal of Econometrics 140: 5-51.

Baltagi BH, Liu L. 2008. Testing for Random Effects and Spatial Lag Dependence in Panel Data Models. Statistics and Probability Letters 78: 3304-3311.

Baxter M, Crucini M. 1993. Explaining Saving-Investment Correlation. American Economic Review 83: 416-436.

Bell KP, Bockstael NE. 2000. Applying the generalized moments estimation approach to spatial problems involving micro-level data. The Review of Economics and Statistics 82: 72-82.

Brueckner JK. 1998. Testing for strategic interaction among local governments: the case of growth controls. Journal of Urban Economics 44: 438-67.

Brueckner JK. 2003. Strategic interaction among governments: An overview of empirical studies. International Regional Science Review 26: 175-188.

Chudik A, Pesaran MH, Tosetti E. 2009. Weak and Strong Cross Section Dependence and Estimation of Large Panels. Mimeo, University of Cambridge.

Cliff AD, Ord JK. 1981. Spatial Processes : Models and Applications. Pion: London.

Coakley J, Kulasi F, Smith R. 1998. The Feldstein-Horioka Puzzle and Capital Mobility: A Review. International Journal of Finance and Economics 3: 169-188.

Conley TG. 1999. GMM Estimation with Cross Sectional Dependence. Journal of Econometrics 92: 1-45.

Conley TG, Ligon E. 2002. Economic distance, spillovers and cross country comparisons. Journal of Economic Growth 7: 157-187.

Conley TG, Topa G. 2002. Socio-economic distance and spatial patterns in unemployment. Journal of Applied Econometrics 17: 303-327.

Corbin A. 2001. Country Specific Effect in the Feldstein-Horioka Paradox: a panel data analysis. Economic Letters 72: 297-302.

Coiteux M, Olivier S. 2000. The Saving Retention Coefficient in the Long Run and in the Short Run: Evidence from Panel Data. Journal of International Money and Finance 19: 535-548.

Dooley M, Frankel J, Mathieson D. 1987. International Capital Mobility: What Do Saving-Investment Correlations Tell Us? IMF Staff Papers 34: 503-530.

Driscoll JC, Kraay AC. 1998. Covariance Matrix Estimation with Spatially Dependent Panel Data. Review of Economics and Statistics 80: 549-560.

Elhorst P. 2001. Dynamic Models in Space and Time. Geographical Analysis 33: 119-140.

Elhorst P. 2003. Specification and Estimation of Spatial Panel Data Models. International Regional Science review 26: 244-268. 
Ertur C, Le Gallo J, Baumont C. 2006. The European Regional Convergence Process, 1980-1995: Do Spatial Regimes and Spatial Dependence Matter? International Regional Science Review 29: 3-34.

Ertur C, Koch W. 2007. Growth, Technological Interdependence and Spatial Externalities: Theory and Evidence. Journal of Applied Econometrics 22:6: 1033-1062.

Ertur, C, Koch W. 2008. A Contribution to the Schumpeterian Growth Theory and Empirics. Document de recherche du LEO.

Feldstein M. 1983. Domestic Saving and International Capital Movements in the Long Run and the Short Run. European Economic Review 21: 139-151.

Feldstein M, Horioka C. 1980. Domestic saving and international capital flows. The Economic Journal 90: 314-329.

Fouquau J, Hurlin C, Rabaud I. 2008. The Feldstein-Horioka puzzle : A Panel Smooth transition regression approach. Economic Modelling 25: 284-299.

Hausman JA. 1978. Specification Tests in Econometrics. Econometrica 46: 12511271.

Jansen WJ. 2000. International Capital Mobility: Evidence from Panel Data. Journal of International Money and Finance 19: 507-511.

Kapoor M, Kelejian HH, Prucha IR. 2007. Panel Data Models with Spatially Autocorrelated Error Components. Journal of Econometrics 140: 97-130.

Kelejian HH, Prucha IR. 1998. A Generalized Spatial Two-Stage Least Squares Procedure for Estimating a Spatial Autoregressive Model with Autoregressive Disturbance. Journal of Real Estate Finance and Economics 17:1: 99-121.

Kelejian HH, Prucha IR. 1999. A Generalized Moments Estimator for the Autoregressive Parameter in a Spatial Model. International Economic Review 40: 509-533.

Kelejian HH, Prucha IR. 2001. On the Asymptotic Distribution of the Moran I Test with Applications. Journal of Econometrics 104: 219-257.

Kelejian HH, Murrell P, Shepotylo O. 2008. Spatial Spillovers in the Development of Institutions. Working Paper. University of Maryland. Available at SSRN: http://ssrn.com/abstract $=1031974$.

Kelejian HH, Tavlas G, Hondroyiannis G. 2006. A Spatial Modelling Approach to Contagion Among Emerging Economies. Open economies review 17: 423Ŭ441.

Klenow PJ, Rodriguez-Clare A. 2005. Externalities and growth. In Handbook of Economic Growth, Aghion P, Durlauf S (eds). Elsevier: Amsterdam.

Krol R. 1996. International Capital Mobility: Evidence from Panel Data. Journal of International Money and Finance 15:3: 467-474.

Le Gallo J, Rey S. 2009. Spatial Analysis of Economic Convergence. In Palgrave Handbook of Econometrics. Volume 2: Applied Econometrics, Mills T, Patterson K (eds). MacMillan: Hampshire. 
Lee LF, Yu J. 2008. Estimation of Spatial Autoregressive Panel Data Models with Fixed Effects. Working Paper. Department of Economics. The Ohio State University.

Lee LF, Yu J. 2009. Some Recent Developments in Spatial Panel Data Models. Working paper. Department of Economics. The Ohio State University.

LeSage J, Pace K. 2009. Introduction to Spatial Econometrics. CRC Press/Taylor and Francis Group: London.

López-Bazo E, Vayá E, Mora A, Suriñach J. 1999. Regional Economic Dynamics and Convergence in the European Union. Annals of Regional Science 33: 343-370.

Murdoch JC, Sandler T, Sargent K. 1997. A tale of two collectives: sulphur versus nitrogen oxides emission reduction in Europe. Economica 64: 281-301.

OECD. 1990. Libéralisation des mouvements de capitaux et des services financiers dans la zone de l'OCDE. OECD.

Nerlove M. 1971. Further Evidences on the Estimation of Dynamic Economic Relations from a Time Series of Cross Sections. Econometrica 39:2: 359-382.

Penati A, Dooley M. 1984. Current Account Imbalances and Capital Formation in Industrial Countries, 1949-1981. IMF Staff Papers 31: 1-24.

Pesaran MH. 2004. General Diagnostic Tests for Cross Section Dependence in Panels. IZA Discussion paper. No. 1240.

Pesaran MH. 2006. Estimation and inference in large heterogeneous panels with a multifactor error structure. Econometrica 74: 967-1012.

Pesaran MH, Tosetti E. 2009. Large Panels with Common Factors and Spatial Correlation. Mimeo. University of Cambridge.

Sinn S. 1992. Saving-Investment Correlations and Capital Mobility: On the evidence from Annual Data. Economic Journal 102: 1162-1170.

Taylor AM. 1996. International Capital Mobility in History: the Saving-Investment Relationship. NBER Working Paper No. 5743.

Tesar L. 1991. Saving, Investment, and International Capital Flows. Journal of International Economics 31: 55-78.

Wooldridge JM. 2002. Econometric Analysis of Cross Section and Panel Data. MIT Press: London.

Yu J, de Jong R, Lee LF. 2008. Quasi-maximum likelihood estimators for spatial dynamic panel data with fixed effects when both $\mathrm{n}$ and $\mathrm{T}$ are large. Journal of Econometrics 146: 118-134. 


\section{A Appendix}

In order to compute the LM statistics, we need to compute FOC and SOC of the general model (7) and evaluate them under the null hypothesis considered.

\section{A.1 FOC}

$$
\frac{\partial L}{\partial \rho}=-(T-1) \operatorname{tr}\left[\mathbf{S}_{n}(\rho)^{-1} \mathbf{W}_{n}\right]+\frac{1}{\sigma^{2}} \sum_{t=1}^{T-1} \mathbf{V}_{n, t}^{*^{\prime}} \mathbf{R}_{n}(\lambda) \mathbf{W}_{n} \mathbf{Y}_{n, t}^{*}
$$

with $\operatorname{tr}()$, the trace operator and $\left.\mathbf{V}_{n, t}^{*}=\mathbf{R}_{n}(\lambda)\right]\left[\mathbf{S}_{n}(\rho) \mathbf{Y}_{n, t}^{*}-\mathbf{X}_{n, t}^{*} \beta\right]$

$$
\begin{aligned}
\frac{\partial L}{\partial \lambda} & =-(T-1) \operatorname{tr}\left[\mathbf{R}_{n}(\lambda)^{-1} \mathbf{M}_{n}\right]+\frac{1}{\sigma^{2}} \sum_{t=1}^{T-1} \mathbf{V}_{n, t}^{*^{\prime}} \mathbf{M}_{n}\left(\mathbf{S}_{n}(\rho) \mathbf{Y}_{n, t}^{*^{\prime}}-\mathbf{X}_{n, t}^{*^{\prime}} \beta\right) \\
\frac{\partial L}{\partial \beta} & =\frac{1}{\sigma^{2}} \sum_{t=1}^{T-1}\left[\mathbf{X}_{n, t}^{*^{\prime}} \mathbf{R}_{n}(\lambda)^{\prime} \mathbf{V}_{n, t}^{*}\right] \\
\frac{\partial L}{\partial \sigma^{2}} & =-\frac{n(T-1)}{2 \sigma^{2}}+\frac{1}{2 \sigma^{4}} \sum_{t=1}^{T-1} \mathbf{V}_{n, t}^{*^{\prime}} \mathbf{V}_{n, t}^{*}
\end{aligned}
$$

We will consider the construction of the joint test. Hence, the null under consideration is $H_{0}: \rho=\lambda=0$, implying $\mathbf{S}_{n}(\rho)=\mathbf{R}_{n}(\lambda)=\mathbf{I}_{n}$. Let us evaluate these FOC under $H_{0}$ to construct the first part of the LM test.

$$
\begin{aligned}
& \left.\frac{\partial L}{\partial \rho}\right|_{H 0}=\frac{1}{\sigma^{2}} \sum_{t=1}^{T-1}\left[\mathbf{Y}_{n, t}^{*}-\mathbf{X}_{n, t}^{*} \beta\right]^{\prime} \mathbf{W}_{n} \mathbf{Y}_{n, t}^{*} \\
& \left.\frac{\partial L}{\partial \lambda}\right|_{H 0}=\frac{1}{\sigma^{2}} \sum_{t=1}^{T-1}\left[\mathbf{Y}_{n, t}^{*}-\mathbf{X}_{n, t}^{*} \beta\right]^{\prime} \mathbf{M}_{n}\left[\mathbf{Y}_{n, t}^{*}-\mathbf{X}_{n, t}^{*} \beta\right]
\end{aligned}
$$

Under the null, we have shown that $\beta$ could be estimated by OLS, using the "pseudo" within transformed model. We will thus replace the unobserved $\mathbf{V}_{n, t}^{*}$ term by the residuals of the OLS estimation of the constrained model, $\tilde{\mathbf{V}}_{n, t}^{*}=\mathbf{Y}_{n, t}^{*}-\mathbf{X}_{n, t}^{*} \tilde{\beta}$. We also define $\tilde{\sigma}^{2}$ as the estimated residual variance of the constrained model. Using this transformation, (41) and (42) constitute the score vector that will be used to define the joint LM test.

We thus define the score vector for $\theta_{1}=[\rho \lambda]^{\prime}$ evaluated under the null as :

$$
\tilde{\mathbf{d}}=\left[\begin{array}{c}
\frac{\sum_{t=1}^{T-1} \tilde{\mathbf{V}}_{n, t}^{\prime^{\prime}} \mathbf{W}_{n} \mathbf{Y}_{n, t}^{*}}{\hat{\sigma}^{2}} \\
\frac{\sum_{t=1}^{T-1} \tilde{\mathbf{V}}_{n, t}^{*^{\prime}} \mathbf{M}_{n} \tilde{\mathbf{V}}_{n, t}^{*}}{\hat{\sigma}^{2}}
\end{array}\right]=\left[\begin{array}{c}
\tilde{R}_{y} \\
\tilde{R}_{v}
\end{array}\right]
$$

We will now go to the derivation of the Hessian matrix and evaluate it under the null hypothesis. 


\section{A.2 SOC}

The second order conditions for the $\theta_{2}=\left[\beta^{\prime} \sigma^{2}\right]^{\prime}$ parameters are :

$$
\begin{aligned}
\frac{\partial^{2} L}{\partial \beta \partial \beta^{\prime}} & =-\frac{\sum_{t=1}^{T-1} \mathbf{X}_{n, t}^{*^{\prime}} \mathbf{R}_{n}(\lambda)^{\prime} \mathbf{R}_{n}(\lambda) \mathbf{X}_{n, t}^{*}}{\sigma^{2}} \\
\frac{\partial^{2} L}{\partial \beta \partial \sigma^{2}} & =-\frac{1}{\sigma^{4}} \sum_{t=1}^{T-1}\left[\mathbf{X}_{n, t}^{*^{\prime}} \mathbf{R}_{n}(\lambda)^{\prime} \mathbf{R}_{n}(\lambda)\left(\mathbf{S}_{n}(\rho) \mathbf{Y}_{n, t}^{*}-\mathbf{X}_{n, t}^{*} \beta\right)\right] \\
\frac{\partial^{2} L}{\partial\left(\sigma^{2}\right)^{2}} & =\frac{n(T-1)}{2 \sigma^{4}}-\frac{\sum_{t=1}^{T-1} \mathbf{V}_{n, t}^{*^{\prime}} \mathbf{V}_{n, t}^{*}}{\sigma^{6}}
\end{aligned}
$$

We will now derive the Information matrix elements for $\theta_{1}$. To achieve it, we will divide this step into three. The reason behind is that we need further information to compute expectations. We will thus calculate first the SOC, find expectations and finally compute the information matrix elements.

$$
\begin{aligned}
& \frac{\partial^{2} L}{\partial(\rho)^{2}}=-(T-1) \operatorname{tr}\left[\left(\mathbf{S}_{n}(\rho)^{-1} \mathbf{W}_{n}\right)^{2}\right] \\
& -\frac{1}{\sigma^{2}} \sum_{t=1}^{T-1}\left(\mathbf{R}_{n}(\lambda) \mathbf{W}_{n} \mathbf{Y}_{n, t}^{*}\right)^{\prime}\left(\mathbf{R}_{n}(\lambda) \mathbf{W}_{n} \mathbf{Y}_{n, t}^{*}\right) \\
& \frac{\partial L^{2}}{\partial \lambda^{2}}=-(T-1) \operatorname{tr}\left[\left(\mathbf{R}_{n}(\lambda)^{-1} \mathbf{M}_{n}\right)^{2}\right] \\
& -\frac{1}{\sigma^{2}} \sum_{t=1}^{T-1}\left[\mathbf{M}_{n}\left[\mathbf{S}_{n}(\rho) \mathbf{Y}_{n, t}^{*}-\mathbf{X}_{n, t}^{*} \beta\right]\right]^{\prime}\left[\mathbf{M}_{n}\left[\mathbf{S}_{n}(\rho) \mathbf{Y}_{n, t}^{*}-\mathbf{X}_{n, t}^{*} \beta\right]\right] \\
& \frac{\partial L^{2}}{\partial \rho \partial \lambda}=-\frac{1}{\sigma^{2}}\left\{\mathbf{M}_{n}\left(\mathbf{S}_{n}(\rho) \mathbf{Y}_{n, t}^{*}-\mathbf{X}_{n, t}^{*} \beta\right)\right\}^{\prime} \mathbf{R}_{n}(\lambda) \mathbf{W}_{n} \mathbf{Y}_{n, t}^{*} \\
& -\frac{1}{\sigma^{2}} \mathbf{V}_{n, t}^{*^{\prime}} \mathbf{M}_{n} \mathbf{W}_{n} \mathbf{Y}_{n, t}^{*} \\
& \frac{\partial L^{2}}{\partial \beta \partial \lambda}=-\frac{1}{\sigma^{2}} \sum_{t=1}^{T-1}\left\{\left[\mathbf{M}_{n} \mathbf{X}_{n, t}^{*}\right]^{\prime} \mathbf{V}_{n, t}^{*}\right\} \\
& -\frac{1}{\sigma^{2}} \sum_{t=1}^{T-1}\left\{\left[\mathbf{R}_{n}(\lambda) \mathbf{X}_{n, t}^{*}\right]^{\prime} \mathbf{M}_{n}\left(\mathbf{S}_{n}(\rho) \mathbf{Y}_{n, t}^{*}-\mathbf{X}_{n, t}^{*} \beta\right)\right\} \\
& \frac{\partial L^{2}}{\partial \beta \partial \rho}=-\frac{1}{\sigma^{2}} \sum_{t=1}^{T-1} \mathbf{X}_{n, t}^{*^{\prime}} \mathbf{R}_{n}(\lambda)^{\prime} \mathbf{R}_{n}(\lambda) \mathbf{W}_{n} \mathbf{Y}_{n, t}^{*} \\
& \frac{\partial L^{2}}{\partial \sigma^{2} \partial \lambda}=-\frac{1}{\sigma^{4}} \sum_{t=1}^{T-1} \mathbf{V}_{n, t}^{*^{\prime}} \mathbf{M}_{n}\left(\mathbf{S}_{n}(\rho) \mathbf{Y}_{n, t}^{*}-\mathbf{X}_{n, t}^{*} \beta\right) \\
& \frac{\partial L^{2}}{\partial \sigma^{2} \partial \rho}=-\frac{1}{\sigma^{4}} \mathbf{V}_{n, t}^{*^{\prime}} \mathbf{R}_{n}(\lambda) \mathbf{W}_{n} \mathbf{Y}_{n, t}^{*}
\end{aligned}
$$


Let us now look at the expectations problem. The within transformation of the general model is written as follow :

$$
\begin{aligned}
& \mathbf{Y}_{n, t}^{*}=\mathbf{S}_{n}(\rho)^{-1} \mathbf{X}_{n, t}^{*} \beta+\mathbf{S}_{n}(\rho)^{-1} \mathbf{U}_{n, t}^{*} \\
&=\mathbf{S}_{n}(\rho)^{-1} \mathbf{X}_{n, t}^{*} \beta+\mathbf{S}_{n}(\rho)^{-1} \mathbf{R}_{n}(\lambda)^{-1} \mathbf{V}_{n, t}^{*} \\
& E\left(\mathbf{U}_{n, t}^{*}\right)=E\left(\mathbf{V}_{n, t}^{*}\right)=0 \\
& E\left(\mathbf{V}_{n, t}^{*} \mathbf{V}_{n, t}^{*^{\prime}}\right)=\sigma^{2} \mathbf{I}_{n(T-1)} \\
& E\left(\mathbf{U}_{n, t}^{*} \mathbf{U}_{n, t}^{*^{\prime}}\right)= \mathbf{R}_{n}(\lambda)^{-1} \sigma^{2} \mathbf{R}_{n}(\lambda)^{-1^{\prime}} \\
& E\left(\mathbf{Y}_{n, t}^{*}\right)= \mathbf{S}_{n}(\rho)^{-1} \mathbf{X}_{n, t}^{*} \beta \\
& E\left(\mathbf{Y}_{n, t}^{*} \mathbf{Y}_{n, t}^{*^{\prime}}\right)=\left(\mathbf{S}_{n}(\rho)^{-1} \mathbf{X}_{n, t}^{*} \beta\right)\left(\mathbf{S}_{n}(\rho)^{-1} \mathbf{X}_{n, t}^{*} \beta\right)^{\prime} \\
& \quad+\mathbf{S}_{n}(\rho)^{-1} \mathbf{R}_{n}(\lambda)^{-1} \sigma^{2}\left(\mathbf{S}_{n}(\rho)^{-1} \mathbf{R}_{n}(\lambda)^{-1}\right)^{\prime}
\end{aligned}
$$

Let now use these results to find the information matrix elements.

$$
\begin{aligned}
I_{\sigma^{2} \lambda} & =-E\left(\frac{\partial^{2} L}{\partial \sigma^{2} \partial \lambda}\right)=\frac{1}{\sigma^{2}}(T-1) \operatorname{tr}\left[\mathbf{M}_{n} \mathbf{R}_{n}(\lambda)^{-1}\right] \\
I_{\sigma^{2} \rho} & =-E\left(\frac{\partial^{2} L}{\partial \sigma^{2} \partial \rho}\right)=\frac{1}{\sigma^{2}}(T-1) \operatorname{tr}\left[\mathbf{R}_{n}(\lambda) \mathbf{W}_{n} \mathbf{S}_{n}(\rho)^{-1} \mathbf{R}_{n}(\lambda)^{-1}\right] \\
\mathbf{I}_{\beta \lambda} & =-E\left(\frac{\partial^{2} L}{\partial \beta \partial \lambda}\right)=\mathbf{0} \\
\mathbf{I}_{\beta \rho} & =-E\left(\frac{\partial^{2} L}{\partial \beta \partial \rho}\right)=\frac{1}{\sigma^{2}} \sum_{t=1}^{T-1} \mathbf{X}_{n, t}^{*^{\prime}} \mathbf{R}_{n}(\lambda)^{\prime} \mathbf{R}_{n}(\lambda) \mathbf{W}_{n} \mathbf{S}_{n}(\rho)^{-1} \mathbf{X}_{n, t}^{*} \beta \\
\mathbf{I}_{\beta \beta^{\prime}} & =-E\left(\frac{\partial L^{2}}{\partial \beta \partial \beta^{\prime}}\right)=\frac{\left(\mathbf{R}_{n}(\lambda) \mathbf{X}_{n, t}^{*}\right)^{\prime}\left(\mathbf{R}_{n}(\lambda) \mathbf{X}_{n, t}^{*}\right)}{\sigma^{2}} \\
\mathbf{I}_{\beta \sigma^{2}} & =-E\left(\frac{\partial L^{2}}{\partial \beta \partial \sigma^{2}}\right)=\mathbf{0} \\
I_{\sigma^{2} \sigma^{2}} & =-E\left(\frac{\partial L^{2}}{\partial \sigma^{2} \partial \sigma^{2}}\right)=\frac{n(T-1)}{2 \sigma^{4}}
\end{aligned}
$$

In order to compute the elements of the $\mathbf{I}_{11}$, we need to use some properties of the trace estimator. For instance, considering $\frac{\partial^{2} L}{\partial \rho^{2}}$, we see that it is formed of two terms. The first is non-stochastic, meaning that it is not affected by the Expectation operator. However, even though the second term is stochastic, it is a scalar. Hence, we can apply a trace operator on it:

$$
\begin{aligned}
& \frac{1}{\sigma^{2}}\left(\mathbf{R}_{n}(\lambda) \mathbf{W}_{n} \mathbf{Y}_{n, t}^{*}\right)^{\prime}\left(\mathbf{R}_{n}(\lambda)\right.\left.\mathbf{W}_{n} \mathbf{Y}_{n, t}^{*}\right)= \\
&=\frac{1}{\sigma^{2}} \operatorname{tr}\left(\left[\left(\mathbf{R}_{n}(\lambda) \mathbf{W}_{n}\right)^{\prime}\left(\mathbf{R}_{n}(\lambda) \mathbf{W}_{n}\right)\left(\mathbf{Y}_{n, t}^{*} \mathbf{Y}_{n, t}^{*^{\prime}}\right)\right]\right.
\end{aligned}
$$

The last expression coming from the cyclic property of the trace operator (commutativity). Taking expectation of (A.31) we get :

$$
\frac{1}{\sigma^{2}} \operatorname{tr}\left[\left(\mathbf{R}_{n}(\lambda) \mathbf{W}_{n}\right)^{\prime}\left(\mathbf{R}_{n}(\lambda) \mathbf{W}_{n} E\left(\mathbf{Y}_{n, t}^{*} \mathbf{Y}_{n, t}^{*^{\prime}}\right)\right)\right]
$$


Replacing the expression of $E\left(\mathbf{Y}_{n, t}^{*} \mathbf{Y}_{n, t}^{*^{\prime}}\right)$ in the above equation allows to find $I_{\rho \rho}$.

$$
\begin{aligned}
I_{\rho \rho}=- & E\left(\frac{\partial^{2} L}{\partial \rho^{2}}\right)=(T-1) \operatorname{tr}\left[\left(\mathbf{S}_{n}(\rho)^{-1} \mathbf{W}_{n}\right)^{2}\right] \\
& +\frac{1}{\sigma^{2}}\left[\sum_{t=1}^{T-1}\left(\mathbf{R}_{n}(\lambda) \mathbf{W}_{n} \mathbf{S}_{n}(\rho)^{-1} \mathbf{X}_{n, t}^{*} \beta\right)^{\prime}\left(\mathbf{R}_{n}(\lambda) \mathbf{W}_{n} \mathbf{S}_{n}(\rho)^{-1} \mathbf{X}_{n, t}^{*} \beta\right)\right] \\
+ & (T-1) \operatorname{tr}\left[\left(\mathbf{R}_{n}(\lambda) \mathbf{W}_{n} \mathbf{S}_{n}(\rho)^{-1} \mathbf{R}_{n}(\lambda)^{-1}\right)^{\prime}\left(\mathbf{R}_{n}(\lambda) \mathbf{W}_{n} \mathbf{S}_{n}(\rho)^{-1} \mathbf{R}_{n}(\lambda)^{-1}\right)\right] \\
I_{\lambda \lambda}=- & E\left(\frac{\partial^{2} L}{\partial \lambda^{2}}\right)=(T-1) \operatorname{tr}\left[\left(\mathbf{R}_{n}(\lambda)^{-1} \mathbf{M}_{n}\right)^{2}\right] \\
& +(T-1) \operatorname{tr}\left[\left(\mathbf{M}_{n} \mathbf{R}_{n}(\lambda)^{-1}\right)^{\prime}\left(\mathbf{M}_{n} \mathbf{R}_{n}(\lambda)^{-1}\right)\right]
\end{aligned}
$$

Finally, using the fact that $E\left(\mathbf{Y}_{n, t}^{*} \mathbf{U}_{n, t}^{*^{\prime}}\right)=\sigma^{2} \mathbf{S}_{n}(\rho)^{-1} \mathbf{R}_{n}(\lambda)^{-1} \mathbf{R}_{n}(\lambda)^{-1^{\prime}}$, we get :

$$
\begin{aligned}
I_{\rho \lambda}=(T-1) \operatorname{tr}\left[\left(\mathbf{M}_{n} \mathbf{R}_{n}(\lambda)^{-1}\right)^{\prime} \mathbf{R}_{n}(\lambda) \mathbf{W}_{n} \mathbf{S}_{n}(\rho)^{-1} \mathbf{R}_{n}(\lambda)^{-1}\right] \\
+(T-1) \operatorname{tr}\left[\mathbf{M}_{n} \mathbf{W}_{n} \mathbf{S}_{n}(\rho)^{-1} \mathbf{R}_{n}(\lambda)^{-1}\right]
\end{aligned}
$$

The final step consists in evaluating all this terms under the null hypothesis of absence of spatial autocorrelation $\left(\mathbf{S}_{n}=\mathbf{R}_{n}=\mathbf{I}_{n}\right)$.

$$
\begin{aligned}
\left.\mathbf{I}_{\beta \beta^{\prime}}\right|_{H 0} & =\frac{\sum_{t=1}^{T-1} \mathbf{X}_{n, t}^{*^{\prime}} \mathbf{X}_{n, t}^{*}}{\sigma^{2}} \\
\left.\mathbf{I}_{\beta \sigma^{2}}\right|_{H 0} & =\mathbf{0} \\
\left.I_{\sigma^{2} \sigma^{2}}\right|_{H 0} & =\frac{n(T-1)}{2 \sigma^{4}} \\
\left.I_{\sigma^{2} \rho}\right|_{H 0} & =0 \\
\left.I_{\sigma^{2} \lambda}\right|_{H 0} & =0 \\
\left.\mathbf{I}_{\beta \lambda}\right|_{H 0} & =\mathbf{0} \\
\left.\mathbf{I}_{\beta \rho}\right|_{H 0} & =\frac{1}{\sigma^{2}} \sum_{t-1}^{T-1} \mathbf{X}_{n, t}^{*^{\prime}} \mathbf{W}_{n} \mathbf{X}_{n, t}^{*} \beta \\
\left.I_{\rho \rho}\right|_{H 0}=(T-1) \operatorname{tr}\left[\left(\mathbf{W}_{n}+W_{n}^{\prime}\right) \mathbf{W}_{n}\right] & +\frac{1}{\sigma^{2}} \sum_{t-1}^{T-1}\left[\left(\mathbf{W}_{n} \mathbf{X}_{n, t}^{*} \beta\right)^{\prime}\left(\mathbf{W}_{n} \mathbf{X}_{n, t}^{*} \beta\right)\right] \\
\left.I_{\rho \lambda}\right|_{H 0} & =(T-1) \operatorname{tr}\left[\left(\mathbf{M}_{n}+\mathbf{M}_{n}^{\prime}\right) \mathbf{W}_{n}\right] \\
\left.I_{\lambda \lambda}\right|_{H 0} & =(T-1) \operatorname{tr}\left[\left(\mathbf{M}_{n}+\mathbf{M}_{n}^{\prime}\right) \mathbf{M}_{n}\right]
\end{aligned}
$$

Let now resume the construction of the LM statistic. We know that the test is written as follows :

$$
L M=\mathbf{d}^{\prime} \mathbf{I}^{11} \mathbf{d} \sim \chi_{q}^{2}
$$


where $q$ is the number of restrictions, $\mathbf{I}^{11}$ is the inverse of the partitioned information matrix and $d$ is the score vector. Using rules of partitioned matrix, we find that

$$
\mathbf{I}^{11}=\left\{\mathbf{I}_{11}-\mathbf{I}_{12} \mathbf{I}^{22} \mathbf{I}_{21}\right\}^{-1}
$$

Let just provide the expression of the different matrices that will be used.

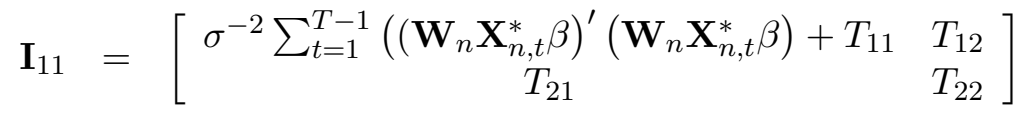

$$
\begin{aligned}
& \mathbf{I}_{12}=\left[\begin{array}{cc}
0 & \sigma^{-2}\left(\mathbf{X}_{n, t}^{* \prime} \mathbf{W}_{n} \mathbf{X}_{n, t}^{*} \beta\right)^{\prime} \\
0 & 0
\end{array}\right] \\
& \mathbf{I}_{21}=\left[\begin{array}{cc}
0 & 0 \\
\sigma^{-2}\left(\mathbf{X}^{\prime} \mathbf{W}_{n}^{*} \mathbf{X} \beta\right) & 0
\end{array}\right] \\
& \mathbf{I}^{22}=\left[\begin{array}{cc}
-2 \sigma^{4} /(n(T-1)) & 0 \\
0 & \sigma^{2}\left(\mathbf{X}_{n, t}^{*^{\prime}} \mathbf{X}_{n, t}^{*}\right)^{-1}
\end{array}\right]
\end{aligned}
$$

with the same notations as in the paper. After some algebra, we find that $\mathbf{I}^{11}=\mathbf{C}^{-1}$, where $\mathbf{C}$ is a $(2 \times 2)$ matrix. For the sake of notation, we chose to present the end of appendix in matrix form. The $\mathbf{C}$ matrix looks like :

$$
\mathbf{C}=\left[\begin{array}{cc}
\sigma^{-2}\left(\mathbf{W}_{n} \mathbf{X}^{*} \beta\right)^{\prime} \mathbf{W}_{n} \mathbf{X}^{*} \beta+T_{11} & T_{12} \\
T_{12} & T_{22}
\end{array}\right]
$$

with $\mathbf{M}_{\mathbf{X}^{*}}=\mathbf{I}_{n(T-1)}-\mathbf{X}^{*}\left(\mathbf{X}^{*^{\prime}} \mathbf{X}^{*}\right)^{-1} \mathbf{X}^{*^{\prime}}$ is the usual annihilator.

Assuming $D=\left(\mathbf{W}_{n} \mathbf{X}^{*} \beta\right)^{\prime} \mathbf{M}_{\mathbf{X}^{*}} \mathbf{W}_{n} \mathbf{X}^{*} \beta$, the determinant of $\mathbf{C}$ is :

$$
Q=|\mathbf{C}|=T_{22}\left(D+T_{11}\right)-T_{12}^{2}
$$

We compute the final test as follows :

$$
\begin{aligned}
L M_{J} & =\frac{1}{Q}\left[\begin{array}{ll}
R_{y} & R_{v}
\end{array}\right]\left[\begin{array}{cc}
T_{22} & -T_{12} \\
-T_{12} & D+T_{11}
\end{array}\right]\left[\begin{array}{c}
R_{y} \\
R_{v}
\end{array}\right] \\
& =\frac{1}{Q}\left[T_{22} R_{y}^{2}-2 T_{12} R_{y} R_{v}+\left(D+T_{11}\right) R_{v}^{2}\right]
\end{aligned}
$$

To implement it, we need to replace the unknown values of $D, Q, R_{y}, R_{v}$ by their estimate, namely $\tilde{D}, \tilde{Q}, \tilde{R}_{y}, \tilde{R}_{v}$ whose values are derived from the estimators of the constrained model, estimated by OLS. The derivation of the four other $L M$ statistics is in the same fashion except that the null is different, implying a (sometimes) different constrained model. 
Table 1: Performance of joint statistics in the Ideal DGP

\begin{tabular}{|c|c|c|c|c|c|c|c|c|c|c|}
\hline \multicolumn{11}{|c|}{$\mathbf{W}=\mathbf{M}, \mathbf{N}=49, \mathrm{~T}=10$} \\
\hline & \multicolumn{2}{|c|}{$\rho=0$} & \multicolumn{2}{|c|}{$\rho=0.1$} & \multicolumn{2}{|c|}{$\rho=0.2$} & \multicolumn{2}{|c|}{$\rho=0.3$} & \multicolumn{2}{|c|}{$\rho=0.4$} \\
\hline$\lambda$ & $L M_{J}$ & $L R_{J}$ & $L M_{J}$ & $L R_{J}$ & $L M_{J}$ & $L R_{J}$ & $L M_{J}$ & $L R_{J}$ & $L M_{J}$ & $L R_{J}$ \\
\hline 0 & 0.042 & 0.035 & 0.285 & 0.276 & 0.84 & 0.832 & 0.993 & 0.992 & 1 & 1 \\
\hline 0.1 & 0.724 & 0.713 & 0.949 & 0.95 & 0.998 & 0.999 & 1 & 1 & 1 & 1 \\
\hline 0.2 & 0.998 & 1 & 1 & 1 & 1 & 1 & 1 & 1 & 1 & 1 \\
\hline 0.3 & 1 & 1 & 1 & 1 & 1 & 1 & 1 & 1 & 1 & 1 \\
\hline \multicolumn{11}{|c|}{$\mathbf{W}=\mathbf{M}, \mathrm{N}=81, \mathrm{~T}=7$} \\
\hline$\lambda$ & $L M_{J}$ & $L R_{J}$ & $L M_{J}$ & $L R_{J}$ & $L M_{J}$ & $L R_{J}$ & $L M_{J}$ & $L R_{J}$ & $L M_{J}$ & $L R_{J}$ \\
\hline 0 & 0.051 & 0.053 & 0.731 & 0.727 & 0.999 & 1 & 1 & 1 & 1 & 1 \\
\hline 0.1 & 0.304 & 0.293 & 0.966 & 0.969 & 1 & 1 & 1 & 1 & 1 & 1 \\
\hline 0.2 & 0.86 & 0.851 & 0.999 & 0.999 & 1 & 1 & 1 & 1 & 1 & 1 \\
\hline 0.3 & 0.999 & 0.998 & 1 & 1 & 1 & 1 & 1 & 1 & 1 & 1 \\
\hline 0.4 & 1 & 1 & 1 & 1 & 1 & 1 & 1 & 1 & 1 & 1 \\
\hline \multicolumn{11}{|c|}{$\mathbf{W} \neq \mathbf{M}, \mathrm{N}=49, \mathrm{~T}=10$} \\
\hline$\lambda$ & $L M_{J}$ & $L R_{J}$ & $L M_{J}$ & $L R_{J}$ & $L M_{J}$ & $L R_{J}$ & $L M_{J}$ & $L R_{J}$ & $L M_{J}$ & $L R_{J}$ \\
\hline 0 & 0.033 & 0.032 & 0.236 & 0.19 & 0.704 & 0.669 & 0.984 & 0.97 & 1 & 1 \\
\hline 0.1 & 0.429 & 0.424 & 0.653 & 0.625 & 0.87 & 0.848 & 0.989 & 0.987 & 1 & 0.999 \\
\hline 0.2 & 0.968 & 0.964 & 0.985 & 0.982 & 0.994 & 0.993 & 1 & 1 & 1 & 1 \\
\hline 0.3 & 1 & 1 & 1 & 1 & 1 & 1 & 1 & 1 & 1 & 1 \\
\hline \multicolumn{11}{|c|}{$\mathbf{W} \neq \mathbf{M}, \mathrm{N}=81, \mathrm{~T}=7$} \\
\hline$\lambda$ & $L M_{J}$ & $L R_{J}$ & $L M_{J}$ & $L R_{J}$ & $L M_{J}$ & $L R_{J}$ & $L M_{J}$ & $L R_{J}$ & $L M_{J}$ & $L R_{J}$ \\
\hline 0 & 0.04 & 0.043 & 0.227 & 0.193 & 0.734 & 0.696 & 0.968 & 0.953 & 1 & 1 \\
\hline 0.1 & 0.435 & 0.43 & 0.61 & 0.585 & 0.886 & 0.868 & 0.986 & 0.984 & 1 & 1 \\
\hline 0.2 & 0.96 & 0.955 & 0.991 & 0.988 & 0.994 & 0.993 & 1 & 1 & 1 & 1 \\
\hline 0.3 & 1 & 1 & 1 & 1 & 1 & 1 & 1 & 1 & 1 & 1 \\
\hline
\end{tabular}

$L M_{J}$ and $L R_{J}$ test the presence of spatial autocorrelation in the sample studied. 
Table 2: Performance of joint statistics in the Robust DGP

\begin{tabular}{|c|c|c|c|c|c|c|c|c|c|c|}
\hline \multicolumn{11}{|c|}{$\mathbf{W}=\mathbf{M}, \mathbf{N}=49, \mathbf{T}=10$} \\
\hline \multirow[b]{2}{*}{$\lambda$} & \multicolumn{2}{|c|}{$\rho=0$} & \multicolumn{2}{|c|}{$\rho=0.1$} & \multicolumn{2}{|c|}{$\rho=0.2$} & \multicolumn{2}{|c|}{$\rho=0.3$} & \multicolumn{2}{|c|}{$\rho=0.4$} \\
\hline & $L M_{J}$ & $L R_{J}$ & $L M_{J}$ & $L R_{J}$ & $L M_{J}$ & $L R_{J}$ & $L M_{J}$ & $L R_{J}$ & $L M_{J}$ & $L R_{J}$ \\
\hline 0 & 0.044 & 0.043 & 0.642 & 0.636 & 1 & 1 & 1 & 1 & 1 & 1 \\
\hline 0.1 & 0.271 & 0.263 & 0.934 & 0.936 & 1 & 1 & 1 & 1 & 1 & 1 \\
\hline 0.2 & 0.839 & 0.826 & 0.999 & 0.999 & 1 & 1 & 1 & 1 & 1 & 1 \\
\hline 0.3 & 0.991 & 0.991 & 1 & 1 & 1 & 1 & 1 & 1 & 1 & 1 \\
\hline 0.4 & 1 & 1 & 1 & 1 & 1 & 1 & 1 & 1 & 1 & 1 \\
\hline \multicolumn{11}{|c|}{$\mathbf{W}=\mathbf{M}, \mathbf{N}=81, \mathrm{~T}=7$} \\
\hline$\lambda$ & $L M_{J}$ & $L R_{J}$ & $L M_{J}$ & $L R_{J}$ & $L M_{J}$ & $L R_{J}$ & $L M_{J}$ & $L R_{J}$ & $L M_{J}$ & $L R_{J}$ \\
\hline 0 & 0.039 & 0.037 & 0.738 & 0.733 & 1 & 1 & 1 & 1 & 1 & 1 \\
\hline 0.1 & 0.304 & 0.308 & 0.963 & 0.966 & 1 & 1 & 1 & 1 & 1 & 1 \\
\hline 0.2 & 0.839 & 0.839 & 1 & 1 & 1 & 1 & 1 & 1 & 1 & 1 \\
\hline 0.3 & 0.998 & 0.998 & 1 & 1 & 1 & 1 & 1 & 1 & 1 & 1 \\
\hline 0.4 & 1 & 1 & 1 & 1 & 1 & 1 & 1 & 1 & 1 & 1 \\
\hline \multicolumn{11}{|c|}{$\mathbf{W} \neq \mathbf{M}, \mathrm{N}=49, \mathrm{~T}=10$} \\
\hline$\lambda$ & $L M_{J}$ & $L R_{J}$ & $L M_{J}$ & $L R_{J}$ & $L M_{J}$ & $L R_{J}$ & $L M_{J}$ & $L R_{J}$ & $L M_{J}$ & $L R_{J}$ \\
\hline 0 & 0.035 & 0.041 & 0.198 & 0.163 & 0.688 & 0.64 & 0.971 & 0.966 & 1 & 0.999 \\
\hline 0.1 & 0.36 & 0.36 & 0.568 & 0.538 & 0.843 & 0.822 & 0.987 & 0.985 & 1 & 1 \\
\hline 0.2 & 0.941 & 0.939 & 0.961 & 0.958 & 0.994 & 0.993 & 0.999 & 0.999 & 1 & 1 \\
\hline 0.3 & 0.999 & 0.999 & 1 & 1 & 1 & 1 & 1 & 1 & 1 & 1 \\
\hline \multicolumn{11}{|c|}{$\mathbf{W} \neq \mathbf{M}, \mathbf{N}=49, \mathrm{~T}=10$} \\
\hline$\lambda$ & $L M_{J}$ & $L R_{J}$ & $L M_{J}$ & $L R_{J}$ & $L M_{J}$ & $L R_{J}$ & $L M_{J}$ & $L R_{J}$ & $L M_{J}$ & $L R_{J}$ \\
\hline 0 & 0.052 & 0.05 & 0.216 & 0.18 & 0.686 & 0.65 & 0.97 & 0.953 & 1 & 0.999 \\
\hline 0.1 & 0.321 & 0.315 & 0.52 & 0.491 & 0.844 & 0.822 & 0.978 & 0.974 & 1 & 1 \\
\hline 0.2 & 0.898 & 0.894 & 0.944 & 0.941 & 0.984 & 0.983 & 0.998 & 0.997 & 1 & 1 \\
\hline 0.3 & 0.999 & 1 & 0.999 & 1 & 1 & 1 & 1 & 1 & 1 & 1 \\
\hline
\end{tabular}

$L M_{J}$ and $L R_{J}$ test the presence of spatial autocorrelation in the sample studied. 
Table 3: Performance of $L M_{\lambda}$ and $L R_{\lambda}$ statistics in the Ideal DGP

\begin{tabular}{|c|c|c|c|c|c|c|c|c|c|c|}
\hline \multicolumn{11}{|c|}{$\mathbf{W}=\mathbf{M}, \mathrm{N}=49, \mathrm{~T}=10$} \\
\hline \multirow[b]{2}{*}{$\rho$} & \multicolumn{2}{|c|}{$\lambda=0$} & \multicolumn{2}{|c|}{$\lambda=0.1$} & \multicolumn{2}{|c|}{$\lambda=0.2$} & \multicolumn{2}{|c|}{$\lambda=0.3$} & \multicolumn{2}{|c|}{$\lambda=0.4$} \\
\hline & $L M_{\lambda}$ & $L R_{\lambda}$ & $L M_{\lambda}$ & $L R_{\lambda}$ & $L M_{\lambda}$ & $L R_{\lambda}$ & $L M_{\lambda}$ & $L R_{\lambda}$ & $L M_{\lambda}$ & $L R_{\lambda}$ \\
\hline 0 & 0.055 & 0.007 & 0.351 & 0.191 & 0.907 & 0.795 & 0.996 & 0.992 & 1 & 1 \\
\hline 0.1 & 0.388 & 0.228 & 0.902 & 0.793 & 0.997 & 0.989 & 1 & 1 & 1 & 1 \\
\hline 0.2 & 0.921 & 0.841 & 0.999 & 0.996 & 1 & 1 & 1 & 1 & 1 & 1 \\
\hline 0.3 & 0.998 & 0.997 & 1 & 1 & 1 & 1 & 1 & 1 & 1 & 1 \\
\hline 0.4 & 1 & 1 & 1 & 1 & 1 & 1 & 1 & 1 & 1 & 1 \\
\hline \multicolumn{11}{|c|}{$\mathbf{W}=\mathbf{M}, \mathrm{N}=81, \mathrm{~T}=7$} \\
\hline$\rho$ & $L M_{\lambda}$ & $L R_{\lambda}$ & $L M_{\lambda}$ & $L R_{\lambda}$ & $L M_{\lambda}$ & $L R_{\lambda}$ & $L M_{\lambda}$ & $L R_{\lambda}$ & $L M_{\lambda}$ & $L R_{\lambda}$ \\
\hline 0 & 0.047 & 0.015 & 0.368 & 0.224 & 0.924 & 0.814 & 0.999 & 0.998 & 1 & 1 \\
\hline 0.1 & 0.359 & 0.21 & 0.906 & 0.813 & 0.998 & 0.995 & 1 & 1 & 1 & 1 \\
\hline 0.2 & 0.912 & 0.839 & 0.997 & 0.994 & 1 & 1 & 1 & 1 & 1 & 1 \\
\hline 0.3 & 1 & 0.997 & 1 & 1 & 1 & 1 & 1 & 1 & 1 & 1 \\
\hline 0.4 & 1 & 1 & 1 & 1 & 1 & 1 & 1 & 1 & 1 & 1 \\
\hline \multicolumn{11}{|c|}{$\mathbf{W} \neq \mathbf{M}, \mathrm{N}=49, \mathrm{~T}=10$} \\
\hline$\rho$ & $L M_{\lambda}$ & $L R_{\lambda}$ & $L M_{\lambda}$ & $L R_{\lambda}$ & $L M_{\lambda}$ & $L R_{\lambda}$ & $L M_{\lambda}$ & $L R_{\lambda}$ & $L M_{\lambda}$ & $L R_{\lambda}$ \\
\hline 0 & 0.05 & 0.012 & 0.246 & 0.112 & 0.788 & 0.646 & 0.977 & 0.941 & 1 & 1 \\
\hline 0.1 & 0.057 & 0.017 & 0.34 & 0.179 & 0.813 & 0.647 & 0.988 & 0.966 & 1 & 0.998 \\
\hline 0.2 & 0.15 & 0.063 & 0.541 & 0.349 & 0.906 & 0.792 & 0.996 & 0.983 & 1 & 1 \\
\hline 0.3 & 0.374 & 0.214 & 0.813 & 0.667 & 0.972 & 0.933 & 1 & 0.998 & 1 & 1 \\
\hline 0.4 & 0.773 & 0.629 & 0.972 & 0.922 & 0.998 & 0.994 & 1 & 1 & 1 & 1 \\
\hline 0.5 & 0.978 & 0.949 & 0.999 & 0.999 & 1 & 1 & 1 & 1 & 1 & 1 \\
\hline 0.6 & 1 & 0.998 & 1 & 1 & 1 & 1 & 1 & 1 & 1 & 1 \\
\hline \multicolumn{11}{|c|}{$\mathbf{W} \neq \mathbf{M}, \mathrm{N}=81, \mathrm{~T}=7$} \\
\hline$\rho$ & $L M_{\lambda}$ & $L R_{\lambda}$ & $L M_{\lambda}$ & $L R_{\lambda}$ & $L M_{\lambda}$ & $L R_{\lambda}$ & $L M_{\lambda}$ & $L R_{\lambda}$ & $L M_{\lambda}$ & $L R_{\lambda}$ \\
\hline 0 & 0.05 & 0.008 & 0.278 & 0.135 & 0.782 & 0.593 & 0.993 & 0.952 & 1 & 1 \\
\hline 0.1 & 0.052 & 0.012 & 0.385 & 0.202 & 0.802 & 0.668 & 0.983 & 0.964 & 1 & 0.999 \\
\hline 0.2 & 0.133 & 0.043 & 0.526 & 0.316 & 0.907 & 0.801 & 0.995 & 0.98 & 1 & 1 \\
\hline 0.3 & 0.38 & 0.216 & 0.822 & 0.662 & 0.98 & 0.954 & 0.998 & 0.993 & 1 & 1 \\
\hline 0.4 & 0.798 & 0.669 & 0.968 & 0.926 & 0.999 & 0.996 & 1 & 1 & 1 & 1 \\
\hline 0.5 & 0.986 & 0.962 & 1 & 0.994 & 1 & 1 & 1 & 1 & 1 & 1 \\
\hline 0.6 & 1 & 1 & 1 & 1 & 1 & 1 & 1 & 1 & 1 & 1 \\
\hline
\end{tabular}

$L M_{\lambda}$ and $L R_{\lambda}$ test for the presence of spatially autocorrelated errors in the specification. 
Table 4: Performance of $L M_{\lambda}$ and $L R_{\lambda}$ statistics in the Robust DGP

\begin{tabular}{|c|c|c|c|c|c|c|c|c|c|c|}
\hline \multicolumn{11}{|c|}{$\mathbf{W}=\mathbf{M}, \mathrm{N}=49, \mathrm{~T}=10$} \\
\hline \multirow[b]{2}{*}{$\rho$} & \multicolumn{2}{|c|}{$\lambda=0$} & \multicolumn{2}{|c|}{$\lambda=0.1$} & \multicolumn{2}{|c|}{$\lambda=0.2$} & \multicolumn{2}{|c|}{$\lambda=0.3$} & \multicolumn{2}{|c|}{$\lambda=0.4$} \\
\hline & $L M_{\lambda}$ & $L R_{\lambda}$ & $L M_{\lambda}$ & $L R_{\lambda}$ & $L M_{\lambda}$ & $L R_{\lambda}$ & $L M_{\lambda}$ & $L R_{\lambda}$ & $L M_{\lambda}$ & $L R_{\lambda}$ \\
\hline 0 & 0.053 & 0.005 & 0.354 & 0.193 & 0.91 & 0.802 & 0.995 & 0.99 & 1 & 1 \\
\hline 0.1 & 0.384 & 0.216 & 0.903 & 0.785 & 0.997 & 0.987 & 1 & 1 & 1 & 1 \\
\hline 0.2 & 0.916 & 0.818 & 0.999 & 0.996 & 1 & 1 & 1 & 1 & 1 & 1 \\
\hline 0.3 & 0.997 & 0.996 & 1 & 1 & 1 & 1 & 1 & 1 & 1 & 1 \\
\hline 0.4 & 1 & 1 & 1 & 1 & 1 & 1 & 1 & 1 & 1 & 1 \\
\hline \multicolumn{11}{|c|}{$\mathbf{W}=\mathbf{M}, \mathrm{N}=81, \mathrm{~T}=7$} \\
\hline$\rho$ & $L M_{\lambda}$ & $L R_{\lambda}$ & $L M_{\lambda}$ & $L R_{\lambda}$ & $L M_{\lambda}$ & $L R_{\lambda}$ & $L M_{\lambda}$ & $L R_{\lambda}$ & $L M_{\lambda}$ & $L R_{\lambda}$ \\
\hline 0 & 0.041 & 0.009 & 0.382 & 0.216 & 0.917 & 0.819 & 1 & 0.996 & 1 & 1 \\
\hline 0.1 & 0.396 & 0.222 & 0.902 & 0.808 & 0.999 & 0.992 & 1 & 1 & 1 & 1 \\
\hline 0.2 & 0.916 & 0.838 & 0.999 & 0.997 & 1 & 1 & 1 & 1 & 1 & 1 \\
\hline 0.3 & 1 & 0.999 & 1 & 1 & 1 & 1 & 1 & 1 & 1 & 1 \\
\hline 0.4 & 1 & 1 & 1 & 1 & 1 & 1 & 1 & 1 & 1 & 1 \\
\hline \multicolumn{11}{|c|}{$\mathbf{W} \neq \mathbf{M}, \mathrm{N}=49, \mathrm{~T}=10$} \\
\hline$\rho$ & $L M_{\lambda}$ & $L R_{\lambda}$ & $L M_{\lambda}$ & $L R_{\lambda}$ & $L M_{\lambda}$ & $L R_{\lambda}$ & $L M_{\lambda}$ & $L R_{\lambda}$ & $L M_{\lambda}$ & $L R_{\lambda}$ \\
\hline 0 & 0.051 & 0.02 & 0.255 & 0.117 & 0.764 & 0.575 & 0.981 & 0.946 & 1 & 0.998 \\
\hline 0.1 & 0.053 & 0.019 & 0.292 & 0.135 & 0.816 & 0.635 & 0.983 & 0.945 & 1 & 1 \\
\hline 0.2 & 0.111 & 0.033 & 0.462 & 0.273 & 0.885 & 0.769 & 0.994 & 0.975 & 1 & 0.999 \\
\hline 0.3 & 0.308 & 0.188 & 0.75 & 0.592 & 0.969 & 0.917 & 1 & 0.996 & 1 & 1 \\
\hline 0.4 & 0.699 & 0.546 & 0.945 & 0.876 & 0.994 & 0.986 & 1 & 1 & 1 & 1 \\
\hline 0.5 & 0.958 & 0.92 & 0.993 & 0.987 & 1 & 0.999 & 1 & 1 & 1 & 1 \\
\hline 0.6 & 1 & 1 & 1 & 1 & 1 & 1 & 1 & 1 & 1 & 1 \\
\hline \multicolumn{11}{|c|}{$\mathbf{W} \neq \mathbf{M}, \mathrm{N}=81, \mathrm{~T}=7$} \\
\hline$\rho$ & $L M_{\lambda}$ & $L R_{\lambda}$ & $L M_{\lambda}$ & $L R_{\lambda}$ & $L M_{\lambda}$ & $L R_{\lambda}$ & $L M_{\lambda}$ & $L R_{\lambda}$ & $L M_{\lambda}$ & $L R_{\lambda}$ \\
\hline 0 & 0.049 & 0.005 & 0.243 & 0.101 & 0.769 & 0.58 & 0.98 & 0.948 & 1 & 0.999 \\
\hline 0.1 & 0.069 & 0.026 & 0.338 & 0.168 & 0.8 & 0.627 & 0.988 & 0.956 & 1 & 1 \\
\hline 0.2 & 0.11 & 0.044 & 0.487 & 0.308 & 0.899 & 0.8 & 0.997 & 0.987 & 1 & 0.999 \\
\hline 0.3 & 0.329 & 0.187 & 0.752 & 0.609 & 0.966 & 0.926 & 1 & 0.994 & 1 & 1 \\
\hline 0.4 & 0.705 & 0.58 & 0.952 & 0.907 & 0.996 & 0.993 & 1 & 0.999 & 1 & 1 \\
\hline 0.5 & 0.977 & 0.954 & 0.998 & 0.993 & 1 & 1 & 1 & 1 & 1 & 1 \\
\hline 0.6 & 1 & 1 & 1 & 1 & 1 & 1 & 1 & 1 & 1 & 1 \\
\hline
\end{tabular}

$L M_{\lambda}$ and $L R_{\lambda}$ test for the presence of spatially autocorrelated errors in the specification. 
Table 5: Performance of $L M_{\rho}$ and $L R_{\rho}$ statistics in the Ideal DGP

\begin{tabular}{|c|c|c|c|c|c|c|c|c|c|c|}
\hline \multirow[b]{3}{*}{$\lambda$} & \multicolumn{10}{|c|}{$\mathbf{W}=\mathbf{M}, \mathbf{N}=49, \mathrm{~T}=10$} \\
\hline & \multicolumn{2}{|c|}{$\rho=0$} & \multicolumn{2}{|c|}{$\rho=0.1$} & \multicolumn{2}{|c|}{$\rho=0.2$} & \multicolumn{2}{|c|}{$\rho=0.3$} & \multicolumn{2}{|c|}{$\rho=0.4$} \\
\hline & $L M_{\rho}$ & $L R_{\rho}$ & $L M_{\rho}$ & $L R_{\rho}$ & $L M_{\rho}$ & $L R_{\rho}$ & $L M_{\rho}$ & $L R_{\rho}$ & $L M_{\rho}$ & $L R_{\rho}$ \\
\hline 0 & 0.048 & 0.008 & 0.822 & 0.666 & 1 & 0.998 & 1 & 1 & 1 & 1 \\
\hline 0.1 & 0.153 & 0.063 & 0.963 & 0.897 & 1 & 1 & 1 & 1 & 1 & 1 \\
\hline 0.2 & 0.484 & 0.315 & 0.996 & 0.981 & 1 & 1 & 1 & 1 & 1 & 1 \\
\hline 0.3 & 0.833 & 0.709 & 0.999 & 0.998 & 1 & 1 & 1 & 1 & 1 & 1 \\
\hline 0.4 & 0.981 & 0.963 & 1 & 1 & 1 & 1 & 1 & 1 & 1 & 1 \\
\hline 0.5 & 0.998 & 0.997 & 1 & 1 & 1 & 1 & 1 & 1 & 1 & 1 \\
\hline 0.6 & 1 & 1 & 1 & 1 & 1 & 1 & 1 & 1 & 1 & 1 \\
\hline \multicolumn{11}{|c|}{$\mathbf{W}=\mathbf{M}, \mathbf{N}=81, \mathrm{~T}=7$} \\
\hline$\lambda$ & $L M_{\rho}$ & $L R_{\rho}$ & $L M_{\rho}$ & $L R_{\rho}$ & $L M_{\rho}$ & $L R_{\rho}$ & $L M_{\rho}$ & $L R_{\rho}$ & $L M_{\rho}$ & $L R_{\rho}$ \\
\hline 0 & 0.063 & 0.019 & 0.815 & 0.673 & 1 & 1 & 1 & 1 & 1 & 1 \\
\hline 0.1 & 0.16 & 0.063 & 0.967 & 0.924 & 1 & 1 & 1 & 1 & 1 & 1 \\
\hline 0.2 & 0.497 & 0.311 & 0.998 & 0.99 & 1 & 1 & 1 & 1 & 1 & 1 \\
\hline 0.3 & 0.838 & 0.705 & 1 & 0.999 & 1 & 1 & 1 & 1 & 1 & 1 \\
\hline 0.4 & 0.983 & 0.955 & 1 & 1 & 1 & 1 & 1 & 1 & 1 & 1 \\
\hline 0.5 & 1 & 0.998 & 1 & 1 & 1 & 1 & 1 & 1 & 1 & 1 \\
\hline \multicolumn{11}{|c|}{$\mathbf{W} \neq \mathbf{M}, \mathbf{N}=49, \mathrm{~T}=10$} \\
\hline$\lambda$ & $L M_{\rho}$ & $L R_{\rho}$ & $L M_{\rho}$ & $L R_{\rho}$ & $L M_{\rho}$ & $L R_{\rho}$ & $L M_{\rho}$ & $L R_{\rho}$ & $L M_{\rho}$ & $L R_{\rho}$ \\
\hline 0 & 0.038 & 0.007 & 0.522 & 0.355 & 0.98 & 0.947 & 1 & 1 & 1 & 1 \\
\hline 0.1 & 0.074 & 0.023 & 0.552 & 0.368 & 0.992 & 0.967 & 1 & 1 & 1 & 1 \\
\hline 0.2 & 0.095 & 0.033 & 0.598 & 0.406 & 0.982 & 0.949 & 1 & 1 & 1 & 1 \\
\hline 0.3 & 0.131 & 0.051 & 0.629 & 0.435 & 0.987 & 0.96 & 1 & 1 & 1 & 1 \\
\hline 0.4 & 0.172 & 0.066 & 0.662 & 0.49 & 0.981 & 0.961 & 1 & 1 & 1 & 1 \\
\hline 0.5 & 0.241 & 0.106 & 0.7 & 0.536 & 0.982 & 0.955 & 1 & 1 & 1 & 1 \\
\hline 0.6 & 0.318 & 0.163 & 0.707 & 0.555 & 0.978 & 0.944 & 0.999 & 0.998 & 1 & 1 \\
\hline 0.7 & 0.442 & 0.258 & 0.727 & 0.587 & 0.968 & 0.942 & 1 & 0.998 & 1 & 1 \\
\hline 0.8 & 0.612 & 0.429 & 0.759 & 0.617 & 0.937 & 0.886 & 1 & 0.999 & 1 & 1 \\
\hline 0.9 & 0.736 & 0.571 & 0.798 & 0.668 & 0.922 & 0.863 & 0.979 & 0.96 & 0.998 & 0.996 \\
\hline \multicolumn{11}{|c|}{$\mathbf{W} \neq \mathbf{M}, \mathbf{N}=49, \mathrm{~T}=10$} \\
\hline$\lambda$ & $L M_{\rho}$ & $L R_{\rho}$ & $L M_{\rho}$ & $L R_{\rho}$ & $L M_{\rho}$ & $L R_{\rho}$ & $L M_{\rho}$ & $L R_{\rho}$ & $L M_{\rho}$ & $L R_{\rho}$ \\
\hline 0 & 0.038 & 0.008 & 0.536 & 0.339 & 0.977 & 0.957 & 1 & 1 & 1 & 1 \\
\hline 0.1 & 0.06 & 0.017 & 0.602 & 0.395 & 0.986 & 0.957 & 1 & 1 & 1 & 1 \\
\hline 0.2 & 0.098 & 0.027 & 0.581 & 0.382 & 0.986 & 0.962 & 1 & 1 & 1 & 1 \\
\hline 0.3 & 0.123 & 0.043 & 0.646 & 0.469 & 0.988 & 0.959 & 1 & 1 & 1 & 1 \\
\hline 0.4 & 0.197 & 0.09 & 0.681 & 0.489 & 0.99 & 0.963 & 1 & 1 & 1 & 1 \\
\hline 0.5 & 0.228 & 0.091 & 0.713 & 0.543 & 0.99 & 0.972 & 1 & 1 & 1 & 1 \\
\hline 0.6 & 0.326 & 0.18 & 0.727 & 0.577 & 0.984 & 0.958 & 1 & 1 & 1 & 1 \\
\hline 0.7 & 0.471 & 0.27 & 0.765 & 0.62 & 0.978 & 0.951 & 1 & 0.999 & 1 & 1 \\
\hline 0.8 & 0.561 & 0.367 & 0.794 & 0.643 & 0.953 & 0.91 & 0.999 & 0.994 & 1 & 1 \\
\hline 0.9 & 0.745 & 0.6 & 0.812 & 0.686 & 0.939 & 0.881 & 0.986 & 0.968 & 0.999 & 0.997 \\
\hline
\end{tabular}


Table 6: Performance of $L M_{\rho}$ and $L R_{\rho}$ statistics in the Robust GDP

\begin{tabular}{|c|c|c|c|c|c|c|c|c|c|c|}
\hline \multicolumn{11}{|c|}{$\mathbf{W}=\mathbf{M}, \mathrm{N}=49, \mathrm{~T}=10$} \\
\hline & \multicolumn{2}{|c|}{$\rho=0$} & \multicolumn{2}{|c|}{$\rho=0.1$} & \multicolumn{2}{|c|}{$\rho=0.2$} & \multicolumn{2}{|c|}{$\rho=0.3$} & \multicolumn{2}{|c|}{$\rho=0.4$} \\
\hline$\lambda$ & $L M_{\rho}$ & $L R_{\rho}$ & $L M_{\rho}$ & $L R_{\rho}$ & $L M_{\rho}$ & $L R_{\rho}$ & $L M_{\rho}$ & $L R_{\rho}$ & $L M_{\rho}$ & $L R_{\rho}$ \\
\hline 0 & 0.038 & 0.007 & 0.522 & 0.355 & 0.98 & 0.947 & 1 & 1 & 1 & 1 \\
\hline 0.1 & 0.248 & 0.13 & 0.92 & 0.803 & 1 & 1 & 1 & 1 & 1 & 1 \\
\hline 0.2 & 0.764 & 0.605 & 0.995 & 0.983 & 1 & 1 & 1 & 1 & 1 & 1 \\
\hline 0.3 & 0.984 & 0.954 & 1 & 1 & 1 & 1 & 1 & 1 & 1 & 1 \\
\hline 0.4 & 1 & 0.999 & 1 & 1 & 1 & 1 & 1 & 1 & 1 & 1 \\
\hline \multicolumn{11}{|c|}{$\mathbf{W}=\mathbf{M}, \mathrm{N}=81, \mathrm{~T}=7$} \\
\hline$\lambda$ & $L M_{\rho}$ & $L R_{\rho}$ & $L M_{\rho}$ & $L R_{\rho}$ & $L M_{\rho}$ & $L R_{\rho}$ & $L M_{\rho}$ & $L R_{\rho}$ & $L M_{\rho}$ & $L R_{\rho}$ \\
\hline 0 & 0.047 & 0.008 & 0.446 & 0.283 & 0.964 & 0.924 & 1 & 0.999 & 1 & 1 \\
\hline 0.1 & 0.306 & 0.162 & 0.923 & 0.821 & 0.999 & 0.997 & 1 & 1 & 1 & 1 \\
\hline 0.2 & 0.862 & 0.727 & 0.998 & 0.993 & 1 & 1 & 1 & 1 & 1 & 1 \\
\hline 0.3 & 0.996 & 0.983 & 1 & 1 & 1 & 1 & 1 & 1 & 1 & 1 \\
\hline 0.4 & 1 & 1 & 1 & 1 & 1 & 1 & 1 & 1 & 1 & 1 \\
\hline \multicolumn{11}{|c|}{$\mathbf{W} \neq \mathbf{M}, \mathbf{N}=49, \mathbf{T}=10$} \\
\hline$\lambda$ & $L M_{\rho}$ & $L R_{\rho}$ & $L M_{\rho}$ & $L R_{\rho}$ & $L M_{\rho}$ & $L R_{\rho}$ & $L M_{\rho}$ & $L R_{\rho}$ & $L M_{\rho}$ & $L R_{\rho}$ \\
\hline 0 & 0.042 & 0.006 & 0.391 & 0.24 & 0.952 & 0.876 & 1 & 1 & 1 & 1 \\
\hline 0.1 & 0.066 & 0.019 & 0.469 & 0.29 & 0.959 & 0.89 & 0.999 & 0.998 & 1 & 1 \\
\hline 0.2 & 0.113 & 0.038 & 0.516 & 0.344 & 0.956 & 0.898 & 0.999 & 0.999 & 1 & 1 \\
\hline 0.3 & 0.132 & 0.055 & 0.574 & 0.392 & 0.968 & 0.918 & 0.999 & 0.999 & 1 & 1 \\
\hline 0.4 & 0.197 & 0.09 & 0.618 & 0.439 & 0.97 & 0.92 & 1 & 1 & 1 & 1 \\
\hline 0.5 & 0.302 & 0.131 & 0.675 & 0.495 & 0.973 & 0.935 & 1 & 1 & 1 & 1 \\
\hline 0.6 & 0.384 & 0.19 & 0.636 & 0.472 & 0.961 & 0.922 & 1 & 0.999 & 1 & 1 \\
\hline 0.7 & 0.489 & 0.302 & 0.716 & 0.554 & 0.961 & 0.919 & 1 & 0.996 & 1 & 1 \\
\hline 0.8 & 0.615 & 0.402 & 0.741 & 0.587 & 0.947 & 0.896 & 0.993 & 0.984 & 1 & 0.999 \\
\hline 0.9 & 0.769 & 0.607 & 0.794 & 0.654 & 0.917 & 0.838 & 0.98 & 0.96 & 0.998 & 0.996 \\
\hline \multicolumn{11}{|c|}{$\mathbf{W} \neq \mathbf{M}, \mathrm{N}=81, \mathrm{~T}=7$} \\
\hline$\lambda$ & $L M_{\rho}$ & $L R_{\rho}$ & $L M_{\rho}$ & $L R_{\rho}$ & $L M_{\rho}$ & $L R_{\rho}$ & $L M_{\rho}$ & $L R_{\rho}$ & $L M_{\rho}$ & $L R_{\rho}$ \\
\hline 0 & 0.047 & 0.008 & 0.446 & 0.283 & 0.964 & 0.924 & 1 & 0.999 & 1 & 1 \\
\hline 0.1 & 0.069 & 0.024 & 0.517 & 0.328 & 0.966 & 0.929 & 1 & 1 & 1 & 1 \\
\hline 0.2 & 0.084 & 0.027 & 0.553 & 0.366 & 0.969 & 0.927 & 1 & 1 & 1 & 1 \\
\hline 0.3 & 0.13 & 0.046 & 0.607 & 0.408 & 0.982 & 0.943 & 0.999 & 0.999 & 1 & 1 \\
\hline 0.4 & 0.214 & 0.083 & 0.626 & 0.446 & 0.979 & 0.942 & 1 & 1 & 1 & 1 \\
\hline 0.5 & 0.265 & 0.119 & 0.647 & 0.463 & 0.981 & 0.943 & 1 & 1 & 1 & 1 \\
\hline 0.6 & 0.345 & 0.18 & 0.678 & 0.512 & 0.968 & 0.938 & 1 & 0.999 & 1 & 1 \\
\hline 0.7 & 0.476 & 0.279 & 0.744 & 0.59 & 0.964 & 0.918 & 1 & 0.998 & 1 & 1 \\
\hline 0.8 & 0.613 & 0.409 & 0.753 & 0.593 & 0.944 & 0.9 & 0.995 & 0.99 & 1 & 1 \\
\hline 0.9 & 0.752 & 0.589 & 0.786 & 0.659 & 0.918 & 0.855 & 0.988 & 0.967 & 1 & 1 \\
\hline
\end{tabular}

$L M_{\rho}$ and $L R_{\rho}$ test for the presence an endogenous spatial lag in the specification. 
Table 7: Performance of $L M_{\lambda \mid \rho}$ and $L R_{\lambda \mid \rho}$ in the Ideal DGP

\begin{tabular}{|c|c|c|c|c|c|c|c|c|c|c|}
\hline \multicolumn{11}{|c|}{$\mathbf{W}=\mathbf{M}, \mathbf{N}=49, \mathrm{~T}=10$} \\
\hline \multirow[b]{2}{*}{$\rho$} & \multicolumn{2}{|c|}{$\lambda=0$} & \multicolumn{2}{|c|}{$\lambda=0.1$} & \multicolumn{2}{|c|}{$\lambda=0.2$} & \multicolumn{2}{|c|}{$\lambda=0.3$} & \multicolumn{2}{|c|}{$\lambda=0.4$} \\
\hline & $L M_{\lambda \mid \rho}$ & $L R_{\lambda \mid \rho}$ & $L M_{\lambda \mid \rho}$ & $L R_{\lambda \mid \rho}$ & $L M_{\lambda \mid \rho}$ & $L R_{\lambda \mid \rho}$ & $L M_{\lambda \mid \rho}$ & $L R_{\lambda \mid \rho}$ & $L M_{\lambda \mid \rho}$ & $L R_{\lambda \mid \rho}$ \\
\hline 0 & 0.046 & 0.048 & 0.257 & 0.246 & 0.751 & 0.747 & 0.979 & 0.967 & 1 & 0.996 \\
\hline 0.1 & 0.05 & 0.043 & 0.243 & 0.253 & 0.799 & 0.803 & 0.978 & 0.977 & 1 & 1 \\
\hline 0.2 & 0.042 & 0.023 & 0.258 & 0.258 & 0.778 & 0.791 & 0.979 & 0.978 & 1 & 1 \\
\hline 0.3 & 0.052 & 0.02 & 0.289 & 0.294 & 0.75 & 0.756 & 0.972 & 0.979 & 1 & 1 \\
\hline 0.4 & 0.052 & 0.02 & 0.263 & 0.265 & 0.773 & 0.784 & 0.982 & 0.983 & 0.998 & 0.999 \\
\hline 0.5 & 0.049 & 0.017 & 0.26 & 0.263 & 0.758 & 0.758 & 0.978 & 0.98 & 1 & 1 \\
\hline 0.6 & 0.051 & 0.018 & 0.296 & 0.29 & 0.772 & 0.785 & 0.968 & 0.971 & 1 & 1 \\
\hline 0.7 & 0.052 & 0.012 & 0.308 & 0.302 & 0.812 & 0.806 & 0.973 & 0.979 & 1 & 1 \\
\hline 0.8 & 0.045 & 0.017 & 0.275 & 0.274 & 0.79 & 0.789 & 0.991 & 0.991 & 1 & 1 \\
\hline 0.9 & 0.056 & 0.025 & 0.311 & 0.287 & 0.819 & 0.824 & 0.992 & 0.993 & 0.999 & 0.999 \\
\hline \multicolumn{11}{|c|}{$\mathbf{W}=\mathbf{M}, \mathrm{N}=81, \mathrm{~T}=7$} \\
\hline$\rho$ & $L M_{\lambda \mid \rho}$ & $L R_{\lambda \mid \rho}$ & $L M_{\lambda \mid \rho}$ & $L R_{\lambda \mid \rho}$ & $L M_{\lambda \mid \rho}$ & $L R_{\lambda \mid \rho}$ & $L M_{\lambda \mid \rho}$ & $L R_{\lambda \mid \rho}$ & $L M_{\lambda \mid \rho}$ & $L R_{\lambda \mid \rho}$ \\
\hline 0 & 0.042 & 0.041 & 0.283 & 0.268 & 0.784 & 0.783 & 0.983 & 0.975 & 1 & 0.998 \\
\hline 0.1 & 0.05 & 0.039 & 0.268 & 0.269 & 0.777 & 0.789 & 0.977 & 0.979 & 0.999 & 1 \\
\hline 0.2 & 0.034 & 0.019 & 0.269 & 0.279 & 0.744 & 0.758 & 0.973 & 0.975 & 1 & 1 \\
\hline 0.3 & 0.058 & 0.019 & 0.301 & 0.309 & 0.765 & 0.783 & 0.975 & 0.98 & 1 & 1 \\
\hline 0.4 & 0.051 & 0.019 & 0.25 & 0.243 & 0.791 & 0.799 & 0.975 & 0.979 & 0.999 & 0.999 \\
\hline 0.5 & 0.043 & 0.015 & 0.28 & 0.282 & 0.801 & 0.808 & 0.977 & 0.979 & 0.998 & 0.999 \\
\hline 0.6 & 0.043 & 0.015 & 0.307 & 0.297 & 0.783 & 0.794 & 0.985 & 0.989 & 0.999 & 0.999 \\
\hline 0.7 & 0.062 & 0.022 & 0.291 & 0.278 & 0.831 & 0.825 & 0.98 & 0.986 & 1 & 1 \\
\hline 0.8 & 0.052 & 0.02 & 0.307 & 0.308 & 0.815 & 0.82 & 0.981 & 0.981 & 1 & 1 \\
\hline 0.9 & 0.049 & 0.032 & 0.329 & 0.326 & 0.838 & 0.841 & 0.991 & 0.993 & 1 & 1 \\
\hline \multicolumn{11}{|c|}{$\mathbf{W} \neq \mathbf{M}, \mathbf{N}=49, \mathrm{~T}=10$} \\
\hline$\rho$ & $L M_{\lambda \mid \rho}$ & $L R_{\lambda \mid \rho}$ & $L M_{\lambda \mid \rho}$ & $L R_{\lambda \mid \rho}$ & $L M_{\lambda \mid \rho}$ & $L R_{\lambda \mid \rho}$ & $L M_{\lambda \mid \rho}$ & $L R_{\lambda \mid \rho}$ & $L M_{\lambda \mid \rho}$ & $L R_{\lambda \mid \rho}$ \\
\hline 0 & 0.047 & 0.05 & 0.219 & 0.202 & 0.779 & 0.763 & 0.971 & 0.967 & 1 & 1 \\
\hline 0.1 & 0.044 & 0.048 & 0.259 & 0.247 & 0.756 & 0.738 & 0.984 & 0.976 & 0.999 & 0.999 \\
\hline 0.2 & 0.063 & 0.044 & 0.252 & 0.234 & 0.761 & 0.744 & 0.98 & 0.98 & 1 & 1 \\
\hline 0.3 & 0.039 & 0.02 & 0.271 & 0.248 & 0.728 & 0.711 & 0.981 & 0.978 & 1 & 1 \\
\hline 0.4 & 0.057 & 0.025 & 0.263 & 0.244 & 0.754 & 0.737 & 0.973 & 0.969 & 1 & 1 \\
\hline 0.5 & 0.041 & 0.015 & 0.245 & 0.222 & 0.762 & 0.736 & 0.976 & 0.973 & 1 & 1 \\
\hline 0.6 & 0.055 & 0.018 & 0.253 & 0.217 & 0.712 & 0.674 & 0.976 & 0.973 & 0.999 & 0.999 \\
\hline 0.7 & 0.045 & 0.019 & 0.223 & 0.19 & 0.727 & 0.704 & 0.962 & 0.959 & 1 & 1 \\
\hline 0.8 & 0.042 & 0.022 & 0.273 & 0.242 & 0.707 & 0.679 & 0.96 & 0.956 & 0.997 & 0.996 \\
\hline 0.9 & 0.049 & 0.021 & 0.245 & 0.192 & 0.687 & 0.663 & 0.949 & 0.941 & 0.998 & 0.998 \\
\hline \multicolumn{11}{|c|}{$\mathbf{W} \neq \mathbf{M}, \mathrm{N}=81, \mathrm{~T}=7$} \\
\hline$\rho$ & $L M_{\lambda \mid \rho}$ & $L R_{\lambda \mid \rho}$ & $L M_{\lambda \mid \rho}$ & $L R_{\lambda \mid \rho}$ & $L M_{\lambda \mid \rho}$ & $L R_{\lambda \mid \rho}$ & $L M_{\lambda \mid \rho}$ & $L R_{\lambda \mid \rho}$ & $L M_{\lambda \mid \rho}$ & $L R_{\lambda \mid \rho}$ \\
\hline 0 & 0.047 & 0.051 & 0.26 & 0.245 & 0.769 & 0.744 & 0.989 & 0.98 & 1 & 0.999 \\
\hline 0.1 & 0.043 & 0.046 & 0.287 & 0.27 & 0.754 & 0.744 & 0.979 & 0.978 & 1 & 1 \\
\hline 0.2 & 0.043 & 0.029 & 0.247 & 0.227 & 0.75 & 0.73 & 0.982 & 0.978 & 1 & 1 \\
\hline 0.3 & 0.048 & 0.023 & 0.272 & 0.247 & 0.785 & 0.757 & 0.977 & 0.975 & 0.999 & 0.999 \\
\hline 0.4 & 0.056 & 0.022 & 0.248 & 0.232 & 0.761 & 0.74 & 0.975 & 0.973 & 0.999 & 0.999 \\
\hline 0.5 & 0.043 & 0.02 & 0.231 & 0.204 & 0.76 & 0.736 & 0.967 & 0.962 & 1 & 1 \\
\hline 0.6 & 0.046 & 0.018 & 0.249 & 0.223 & 0.73 & 0.709 & 0.968 & 0.963 & 1 & 1 \\
\hline 0.7 & 0.043 & 0.019 & 0.265 & 0.238 & 0.739 & 0.706 & 0.97 & 0.966 & 0.999 & 0.999 \\
\hline 0.8 & 0.047 & 0.013 & 0.242 & 0.204 & 0.701 & 0.67 & 0.965 & 0.957 & 0.998 & 1 \\
\hline 0.9 & 0.04 & 0.016 & 0.224 & 0.166 & 0.67 & 0.646 & 0.954 & 0.948 & 0.999 & 0.999 \\
\hline
\end{tabular}

$L M_{\lambda \mid \rho}$ and $L R_{\lambda \mid \rho}$ test for the presence of spatially correlated errors when an endogenous spatial lag is included in the specification. 
Table 8: Performance of $L M_{\lambda \mid \rho}$ and $L R_{\lambda \mid \rho}$ in the Robust DGP

\begin{tabular}{|c|c|c|c|c|c|c|c|c|c|c|}
\hline \multicolumn{11}{|c|}{$\mathbf{W}=\mathbf{M}, \mathbf{N}=49, \mathrm{~T}=10$} \\
\hline \multirow[b]{2}{*}{$\rho$} & \multicolumn{2}{|c|}{$\lambda=0$} & \multicolumn{2}{|c|}{$\lambda=0.1$} & \multicolumn{2}{|c|}{$\lambda=0.2$} & \multicolumn{2}{|c|}{$\lambda=0.3$} & \multicolumn{2}{|c|}{$\lambda=0.4$} \\
\hline & $L M_{\lambda \mid \rho}$ & $L R_{\lambda \mid \rho}$ & $L M_{\lambda \mid \rho}$ & $L R_{\lambda \mid \rho}$ & $L M_{\lambda \mid \rho}$ & $L R_{\lambda \mid \rho}$ & $L M_{\lambda \mid \rho}$ & $L R_{\lambda \mid \rho}$ & $L M_{\lambda \mid \rho}$ & $L R_{\lambda \mid \rho}$ \\
\hline 0 & 0.047 & 0.052 & 0.231 & 0.23 & 0.69 & 0.688 & 0.963 & 0.941 & 0.999 & 0.997 \\
\hline 0.1 & 0.057 & 0.05 & 0.255 & 0.26 & 0.717 & 0.722 & 0.963 & 0.968 & 0.999 & 1 \\
\hline 0.2 & 0.053 & 0.023 & 0.262 & 0.275 & 0.706 & 0.723 & 0.969 & 0.975 & 1 & 1 \\
\hline 0.3 & 0.055 & 0.014 & 0.273 & 0.279 & 0.746 & 0.751 & 0.975 & 0.976 & 0.999 & 0.999 \\
\hline 0.4 & 0.049 & 0.024 & 0.237 & 0.239 & 0.785 & 0.8 & 0.981 & 0.985 & 1 & 1 \\
\hline 0.5 & 0.039 & 0.012 & 0.278 & 0.285 & 0.772 & 0.788 & 0.981 & 0.986 & 0.999 & 0.999 \\
\hline 0.6 & 0.054 & 0.03 & 0.302 & 0.3 & 0.805 & 0.817 & 0.984 & 0.987 & 1 & 1 \\
\hline 0.7 & 0.06 & 0.041 & 0.306 & 0.297 & 0.806 & 0.814 & 0.99 & 0.991 & 1 & 1 \\
\hline 0.8 & 0.052 & 0.034 & 0.318 & 0.327 & 0.832 & 0.841 & 0.993 & 0.993 & 1 & 1 \\
\hline 0.9 & 0.052 & 0.035 & 0.357 & 0.346 & 0.87 & 0.87 & 0.997 & 0.998 & 1 & 1 \\
\hline \multicolumn{11}{|c|}{$\mathbf{W}=\mathbf{M}, \mathrm{N}=81, \mathrm{~T}=7$} \\
\hline$\rho$ & $L M_{\lambda \mid \rho}$ & $L R_{\lambda \mid \rho}$ & $L M_{\lambda \mid \rho}$ & $L R_{\lambda \mid \rho}$ & $L M_{\lambda \mid \rho}$ & $L R_{\lambda \mid \rho}$ & $L M_{\lambda \mid \rho}$ & $L R_{\lambda \mid \rho}$ & $L M_{\lambda \mid \rho}$ & $L R_{\lambda \mid \rho}$ \\
\hline 0 & 0.048 & 0.045 & 0.268 & 0.264 & 0.745 & 0.74 & 0.981 & 0.97 & 0.999 & 0.999 \\
\hline 0.1 & 0.06 & 0.05 & 0.258 & 0.259 & 0.783 & 0.793 & 0.979 & 0.986 & 1 & 1 \\
\hline 0.2 & 0.056 & 0.032 & 0.26 & 0.27 & 0.788 & 0.797 & 0.988 & 0.99 & 1 & 1 \\
\hline 0.3 & 0.04 & 0.016 & 0.281 & 0.286 & 0.803 & 0.814 & 0.992 & 0.992 & 1 & 1 \\
\hline 0.4 & 0.047 & 0.018 & 0.28 & 0.285 & 0.812 & 0.833 & 0.99 & 0.993 & 1 & 1 \\
\hline 0.5 & 0.055 & 0.027 & 0.274 & 0.283 & 0.844 & 0.847 & 0.994 & 0.997 & 1 & 1 \\
\hline 0.6 & 0.05 & 0.031 & 0.335 & 0.334 & 0.818 & 0.824 & 0.993 & 0.994 & 1 & 1 \\
\hline 0.7 & 0.051 & 0.034 & 0.322 & 0.323 & 0.847 & 0.85 & 0.995 & 0.996 & 1 & 1 \\
\hline 0.8 & 0.05 & 0.03 & 0.342 & 0.36 & 0.862 & 0.869 & 0.999 & 0.996 & 1 & 1 \\
\hline 0.9 & 0.05 & 0.043 & 0.367 & 0.368 & 0.888 & 0.881 & 0.997 & 0.997 & 1 & 1 \\
\hline \multicolumn{11}{|c|}{$\mathbf{W} \neq \mathbf{M}, \mathbf{N}=49, \mathrm{~T}=10$} \\
\hline$\rho$ & $L M_{\lambda \mid \rho}$ & $L R_{\lambda \mid \rho}$ & $L M_{\lambda \mid \rho}$ & $L R_{\lambda \mid \rho}$ & $L M_{\lambda \mid \rho}$ & $L R_{\lambda \mid \rho}$ & $L M_{\lambda \mid \rho}$ & $L R_{\lambda \mid \rho}$ & $L M_{\lambda \mid \rho}$ & $L R_{\lambda \mid \rho}$ \\
\hline 0 & 0.051 & 0.054 & 0.23 & 0.209 & 0.74 & 0.712 & 0.977 & 0.972 & 0.999 & 0.998 \\
\hline 0.1 & 0.057 & 0.059 & 0.225 & 0.215 & 0.751 & 0.734 & 0.97 & 0.969 & 0.999 & 0.999 \\
\hline 0.2 & 0.04 & 0.026 & 0.222 & 0.202 & 0.744 & 0.73 & 0.983 & 0.98 & 1 & 1 \\
\hline 0.3 & 0.05 & 0.025 & 0.238 & 0.218 & 0.754 & 0.736 & 0.973 & 0.968 & 1 & 1 \\
\hline 0.4 & 0.057 & 0.034 & 0.216 & 0.199 & 0.695 & 0.676 & 0.977 & 0.976 & 1 & 1 \\
\hline 0.5 & 0.057 & 0.031 & 0.224 & 0.202 & 0.735 & 0.711 & 0.964 & 0.963 & 0.999 & 0.999 \\
\hline 0.6 & 0.06 & 0.02 & 0.223 & 0.192 & 0.72 & 0.698 & 0.973 & 0.97 & 1 & 0.999 \\
\hline 0.7 & 0.055 & 0.035 & 0.235 & 0.203 & 0.707 & 0.677 & 0.967 & 0.963 & 0.999 & 0.998 \\
\hline 0.8 & 0.048 & 0.025 & 0.226 & 0.21 & 0.698 & 0.675 & 0.951 & 0.948 & 1 & 1 \\
\hline 0.9 & 0.042 & 0.022 & 0.234 & 0.19 & 0.697 & 0.664 & 0.957 & 0.944 & 0.996 & 0.997 \\
\hline \multicolumn{11}{|c|}{$\mathbf{W} \neq \mathbf{M}, \mathrm{N}=81, \mathrm{~T}=7$} \\
\hline$\rho$ & $L M_{\lambda \mid \rho}$ & $L R_{\lambda \mid \rho}$ & $L M_{\lambda \mid \rho}$ & $L R_{\lambda \mid \rho}$ & $L M_{\lambda \mid \rho}$ & $L R_{\lambda \mid \rho}$ & $L M_{\lambda \mid \rho}$ & $L R_{\lambda \mid \rho}$ & $L M_{\lambda \mid \rho}$ & $L R_{\lambda \mid \rho}$ \\
\hline 0 & 0.048 & 0.047 & 0.218 & 0.195 & 0.753 & 0.722 & 0.977 & 0.972 & 1 & 1 \\
\hline 0.1 & 0.064 & 0.066 & 0.26 & 0.241 & 0.745 & 0.731 & 0.98 & 0.98 & 1 & 1 \\
\hline 0.2 & 0.046 & 0.03 & 0.239 & 0.227 & 0.755 & 0.747 & 0.982 & 0.978 & 0.999 & 0.999 \\
\hline 0.3 & 0.057 & 0.022 & 0.237 & 0.217 & 0.74 & 0.726 & 0.982 & 0.978 & 1 & 1 \\
\hline 0.4 & 0.042 & 0.03 & 0.257 & 0.235 & 0.752 & 0.73 & 0.973 & 0.968 & 1 & 1 \\
\hline 0.5 & 0.04 & 0.018 & 0.217 & 0.187 & 0.726 & 0.702 & 0.983 & 0.976 & 1 & 1 \\
\hline 0.6 & 0.052 & 0.022 & 0.247 & 0.214 & 0.749 & 0.717 & 0.972 & 0.969 & 0.999 & 0.999 \\
\hline 0.7 & 0.055 & 0.041 & 0.255 & 0.217 & 0.731 & 0.703 & 0.964 & 0.958 & 1 & 1 \\
\hline 0.8 & 0.051 & 0.035 & 0.219 & 0.198 & 0.711 & 0.692 & 0.959 & 0.953 & 0.999 & 0.999 \\
\hline 0.9 & 0.05 & 0.026 & 0.225 & 0.185 & 0.704 & 0.678 & 0.965 & 0.955 & 1 & 1 \\
\hline
\end{tabular}

$L M_{\lambda \mid \rho}$ and $L R_{\lambda \mid \rho}$ test for the presence of spatially correlated errors when an endogenous spatial lag is included in the specification. 


\begin{tabular}{|c|c|c|c|c|c|c|c|c|}
\hline \multicolumn{9}{|c|}{$\mathbf{W}=\mathbf{M}, \mathbf{N}=49, \mathrm{~T}=10$} \\
\hline & \multicolumn{2}{|c|}{$\rho=0$} & \multicolumn{2}{|c|}{$\rho=0.1$} & \multicolumn{2}{|c|}{$\rho=0.2$} & \multicolumn{2}{|c|}{$\rho=0.3$} \\
\hline$\lambda$ & $L M_{\rho \mid \lambda}$ & $L R_{\rho \mid \lambda}$ & $L M_{\rho \mid \lambda}$ & $L R_{\rho \mid \lambda}$ & $L M_{\rho \mid \lambda}$ & $L R_{\rho \mid \lambda}$ & $L M_{\rho \mid \lambda}$ & $L R_{\rho \mid \lambda}$ \\
\hline 0 & 0.046 & 0.046 & 0.625 & 0.618 & 0.991 & 0.99 & 1 & 1 \\
\hline 0.1 & 0.063 & 0.061 & 0.601 & 0.622 & 0.993 & 0.995 & 1 & 1 \\
\hline 0.2 & 0.057 & 0.051 & 0.583 & 0.603 & 0.985 & 0.991 & 1 & 1 \\
\hline 0.3 & 0.046 & 0.023 & 0.616 & 0.597 & 0.986 & 0.989 & 1 & 1 \\
\hline 0.4 & 0.058 & 0.022 & 0.577 & 0.594 & 0.981 & 0.984 & 1 & 1 \\
\hline 0.5 & 0.063 & 0.009 & 0.54 & 0.579 & 0.987 & 0.991 & 1 & 1 \\
\hline 0.6 & 0.04 & 0.008 & 0.612 & 0.584 & 0.988 & 0.987 & 1 & 1 \\
\hline 0.7 & 0.054 & 0.02 & 0.545 & 0.554 & 0.979 & 0.981 & 1 & 1 \\
\hline 0.8 & 0.043 & 0.022 & 0.538 & 0.565 & 0.981 & 0.983 & 1 & 1 \\
\hline 0.9 & 0.048 & 0.019 & 0.606 & 0.588 & 0.99 & 0.991 & 1 & 0.999 \\
\hline \multicolumn{9}{|c|}{$\mathbf{W}=\mathbf{M}, \mathrm{N}=81, \mathrm{~T}=7$} \\
\hline$\lambda$ & $L M_{\rho \mid \lambda}$ & $L R_{\rho \mid \lambda}$ & $L M_{\rho \mid \lambda}$ & $L R_{\rho \mid \lambda}$ & $L M_{\rho \mid \lambda}$ & $L R_{\rho \mid \lambda}$ & $L M_{\rho \mid \lambda}$ & $L R_{\rho \mid \lambda}$ \\
\hline 0 & 0.054 & 0.055 & 0.655 & 0.645 & 0.998 & 0.993 & 1 & 1 \\
\hline 0.1 & 0.05 & 0.045 & 0.646 & 0.66 & 0.992 & 0.992 & 1 & 1 \\
\hline 0.2 & 0.057 & 0.05 & 0.606 & 0.621 & 0.991 & 0.994 & 1 & 1 \\
\hline 0.3 & 0.051 & 0.026 & 0.66 & 0.644 & 0.988 & 0.99 & 1 & 1 \\
\hline 0.4 & 0.057 & 0.03 & 0.602 & 0.613 & 0.993 & 0.993 & 1 & 1 \\
\hline 0.5 & 0.059 & 0.017 & 0.578 & 0.619 & 0.985 & 0.988 & 1 & 1 \\
\hline 0.6 & 0.049 & 0.016 & 0.644 & 0.613 & 0.988 & 0.987 & 1 & 1 \\
\hline 0.7 & 0.052 & 0.011 & 0.591 & 0.61 & 0.983 & 0.988 & 1 & 1 \\
\hline 0.8 & 0.042 & 0.02 & 0.578 & 0.6 & 0.992 & 0.992 & 1 & 1 \\
\hline 0.9 & 0.048 & 0.019 & 0.671 & 0.654 & 0.996 & 0.997 & 1 & 1 \\
\hline \multicolumn{9}{|c|}{$\mathbf{W} \neq \mathbf{M}, \mathrm{N}=49, \mathrm{~T}=10$} \\
\hline$\lambda$ & $L M_{\rho \mid \lambda}$ & $L R_{\rho \mid \lambda}$ & $L M_{\rho \mid \lambda}$ & $L R_{\rho \mid \lambda}$ & $L M_{\rho \mid \lambda}$ & $L R_{\rho \mid \lambda}$ & $L M_{\rho \mid \lambda}$ & $L R_{\rho \mid \lambda}$ \\
\hline 0 & 0.038 & 0.036 & 0.519 & 0.511 & 0.982 & 0.978 & 1 & 0.999 \\
\hline 0.1 & 0.057 & 0.058 & 0.492 & 0.501 & 0.989 & 0.989 & 1 & 1 \\
\hline 0.2 & 0.049 & 0.052 & 0.479 & 0.494 & 0.964 & 0.965 & 1 & 1 \\
\hline 0.3 & 0.045 & 0.036 & 0.46 & 0.465 & 0.966 & 0.97 & 1 & 1 \\
\hline 0.4 & 0.033 & 0.026 & 0.473 & 0.483 & 0.964 & 0.965 & 1 & 1 \\
\hline 0.5 & 0.056 & 0.028 & 0.449 & 0.458 & 0.962 & 0.963 & 1 & 1 \\
\hline 0.6 & 0.044 & 0.027 & 0.45 & 0.455 & 0.961 & 0.963 & 1 & 1 \\
\hline 0.7 & 0.052 & 0.029 & 0.425 & 0.439 & 0.962 & 0.965 & 1 & 1 \\
\hline 0.8 & 0.041 & 0.031 & 0.416 & 0.424 & 0.946 & 0.949 & 1 & 1 \\
\hline 0.9 & 0.046 & 0.036 & 0.429 & 0.433 & 0.959 & 0.957 & 1 & 1 \\
\hline \multicolumn{9}{|c|}{$\mathbf{W} \neq \mathbf{M}, \mathbf{N}=81, \mathrm{~T}=7$} \\
\hline$\lambda$ & $L M_{\rho \mid \lambda}$ & $L R_{\rho \mid \lambda}$ & $L M_{\rho \mid \lambda}$ & $L R_{\rho \mid \lambda}$ & $L M_{\rho \mid \lambda}$ & $L R_{\rho \mid \lambda}$ & $L M_{\rho \mid \lambda}$ & $L R_{\rho \mid \lambda}$ \\
\hline 0 & 0.035 & 0.035 & 0.527 & 0.519 & 0.978 & 0.973 & 1 & 1 \\
\hline 0.1 & 0.047 & 0.049 & 0.534 & 0.545 & 0.976 & 0.981 & 1 & 1 \\
\hline 0.2 & 0.042 & 0.045 & 0.478 & 0.489 & 0.972 & 0.973 & 1 & 1 \\
\hline 0.3 & 0.033 & 0.025 & 0.506 & 0.506 & 0.971 & 0.971 & 1 & 1 \\
\hline 0.4 & 0.051 & 0.045 & 0.492 & 0.504 & 0.971 & 0.973 & 1 & 1 \\
\hline 0.5 & 0.038 & 0.024 & 0.497 & 0.51 & 0.976 & 0.98 & 1 & 1 \\
\hline 0.6 & 0.06 & 0.03 & 0.504 & 0.512 & 0.979 & 0.979 & 1 & 1 \\
\hline 0.7 & 0.046 & 0.028 & 0.461 & 0.475 & 0.965 & 0.967 & 1 & 1 \\
\hline 0.8 & 0.04 & 0.026 & 0.487 & 0.495 & 0.969 & 0.972 & 1 & 1 \\
\hline 0.9 & 0.049 & 0.031 & 0.465 & 0.471 & 0.964 & 0.964 & 1 & 1 \\
\hline
\end{tabular}

$L M_{\rho \mid \lambda}$ and $L R_{\rho \mid \lambda}$ test for the presence an endogenous spatial lag when spatially autocorrelated errors are included in the specification. 
Table 10: Performance of $L M_{\rho \mid \lambda}$ and $L R_{\rho \mid \lambda}$ in the Robust DGP with $\mathbf{W} \neq \mathbf{M}$ $\mathrm{N}=49, \mathrm{~T}=10$

\begin{tabular}{cccccccccccc}
\multicolumn{4}{c}{$\rho=0$} & \multicolumn{2}{c}{$\rho=0.1$} & \multicolumn{2}{c}{$\rho=0.2$} & \multicolumn{2}{c}{$\rho=0.3$} & \multicolumn{2}{c}{$\rho=0.4$} \\
$\lambda$ & $L M_{\rho \mid \lambda}$ & $L R_{\rho \mid \lambda}$ & $L M_{\rho \mid \lambda}$ & $L R_{\rho \mid \lambda}$ & $L M_{\rho \mid \lambda}$ & $L R_{\rho \mid \lambda}$ & $L M_{\rho \mid \lambda}$ & $L R_{\rho \mid \lambda}$ & $L M_{\rho \mid \lambda}$ & $L R_{\rho \mid \lambda}$ \\
\hline 0 & $\mathbf{0 . 0 4 1}$ & $\mathbf{0 . 0 4 6}$ & 0.398 & 0.385 & 0.953 & 0.948 & 1 & 1 & 1 & 1 \\
0.1 & $\mathbf{0 . 0 5 2}$ & $\mathbf{0 . 0 5 5}$ & 0.414 & 0.432 & 0.942 & 0.946 & 0.999 & 0.999 & 1 & 1 \\
0.2 & $\mathbf{0 . 0 5 1}$ & $\mathbf{0 . 0 5}$ & 0.398 & 0.411 & 0.926 & 0.933 & 0.999 & 0.999 & 1 & 1 \\
0.3 & $\mathbf{0 . 0 6}$ & $\mathbf{0 . 0 4 3}$ & 0.414 & 0.416 & 0.936 & 0.942 & 0.999 & 0.999 & 1 & 1 \\
0.4 & $\mathbf{0 . 0 4 1}$ & $\mathbf{0 . 0 3 6}$ & 0.398 & 0.406 & 0.924 & 0.929 & 1 & 1 & 1 & 1 \\
0.5 & $\mathbf{0 . 0 4 5}$ & $\mathbf{0 . 0 1 8}$ & 0.409 & 0.425 & 0.94 & 0.942 & 1 & 1 & 1 & 1 \\
0.6 & $\mathbf{0 . 0 5 3}$ & $\mathbf{0 . 0 2 8}$ & 0.376 & 0.38 & 0.912 & 0.912 & 0.999 & 0.999 & 1 & 1 \\
0.7 & $\mathbf{0 . 0 6}$ & $\mathbf{0 . 0 5 3}$ & 0.359 & 0.373 & 0.912 & 0.92 & 1 & 1 & 1 & 1 \\
0.8 & $\mathbf{0 . 0 4 7}$ & $\mathbf{0 . 0 4 6}$ & 0.371 & 0.377 & 0.903 & 0.909 & 0.999 & 0.999 & 1 & 1 \\
0.9 & $\mathbf{0 . 0 3 9}$ & $\mathbf{0 . 0 3 3}$ & 0.346 & 0.355 & 0.915 & 0.909 & 1 & 0.999 & 1 & 1 \\
\hline & & & & & $\mathrm{N}=81, \mathrm{~T}=7$ & & & & \\
$\lambda$ & $L M_{\rho \mid \lambda}$ & $L R_{\rho \mid \lambda}$ & $L M_{\rho \mid \lambda}$ & $L R_{\rho \mid \lambda}$ & $L M_{\rho \mid \lambda}$ & $L R_{\rho \mid \lambda}$ & $L M_{\rho \mid \lambda}$ & $L R_{\rho \mid \lambda}$ & $L M_{\rho \mid \lambda}$ & $L R_{\rho \mid \lambda}$ \\
\hline 0 & $\mathbf{0 . 0 4 7}$ & $\mathbf{0 . 0 5 1}$ & 0.432 & 0.434 & 0.968 & 0.964 & 1 & 1 & 1 & 1 \\
0.1 & $\mathbf{0 . 0 4 7}$ & $\mathbf{0 . 0 4 7}$ & 0.448 & 0.459 & 0.955 & 0.958 & 1 & 1 & 1 & 1 \\
0.2 & $\mathbf{0 . 0 4 2}$ & $\mathbf{0 . 0 4 4}$ & 0.431 & 0.441 & 0.949 & 0.951 & 1 & 1 & 1 & 1 \\
0.3 & $\mathbf{0 . 0 4 7}$ & $\mathbf{0 . 0 3 1}$ & 0.433 & 0.446 & 0.963 & 0.962 & 0.999 & 0.999 & 1 & 1 \\
0.4 & $\mathbf{0 . 0 4 7}$ & $\mathbf{0 . 0 4}$ & 0.424 & 0.433 & 0.937 & 0.939 & 1 & 1 & 1 & 1 \\
0.5 & $\mathbf{0 . 0 4 5}$ & $\mathbf{0 . 0 2}$ & 0.402 & 0.407 & 0.949 & 0.955 & 1 & 1 & 1 & 1 \\
0.6 & $\mathbf{0 . 0 5 4}$ & $\mathbf{0 . 0 3 1}$ & 0.405 & 0.408 & 0.946 & 0.949 & 1 & 1 & 1 & 1 \\
0.7 & $\mathbf{0 . 0 4 6}$ & $\mathbf{0 . 0 3 9}$ & 0.406 & 0.414 & 0.933 & 0.934 & 1 & 1 & 1 & 1 \\
0.8 & $\mathbf{0 . 0 4 5}$ & $\mathbf{0 . 0 3 9}$ & 0.399 & 0.406 & 0.932 & 0.936 & 1 & 1 & 1 & 1 \\
0.9 & $\mathbf{0 . 0 5}$ & $\mathbf{0 . 0 4 7}$ & 0.406 & 0.407 & 0.936 & 0.932 & 1 & 1 & 1 & 1 \\
\hline
\end{tabular}

$L M_{\rho \mid \lambda}$ and $L R_{\rho \mid \lambda}$ test for the presence an endogenous spatial lag when spatially autocorrelated errors are included in the specification. 


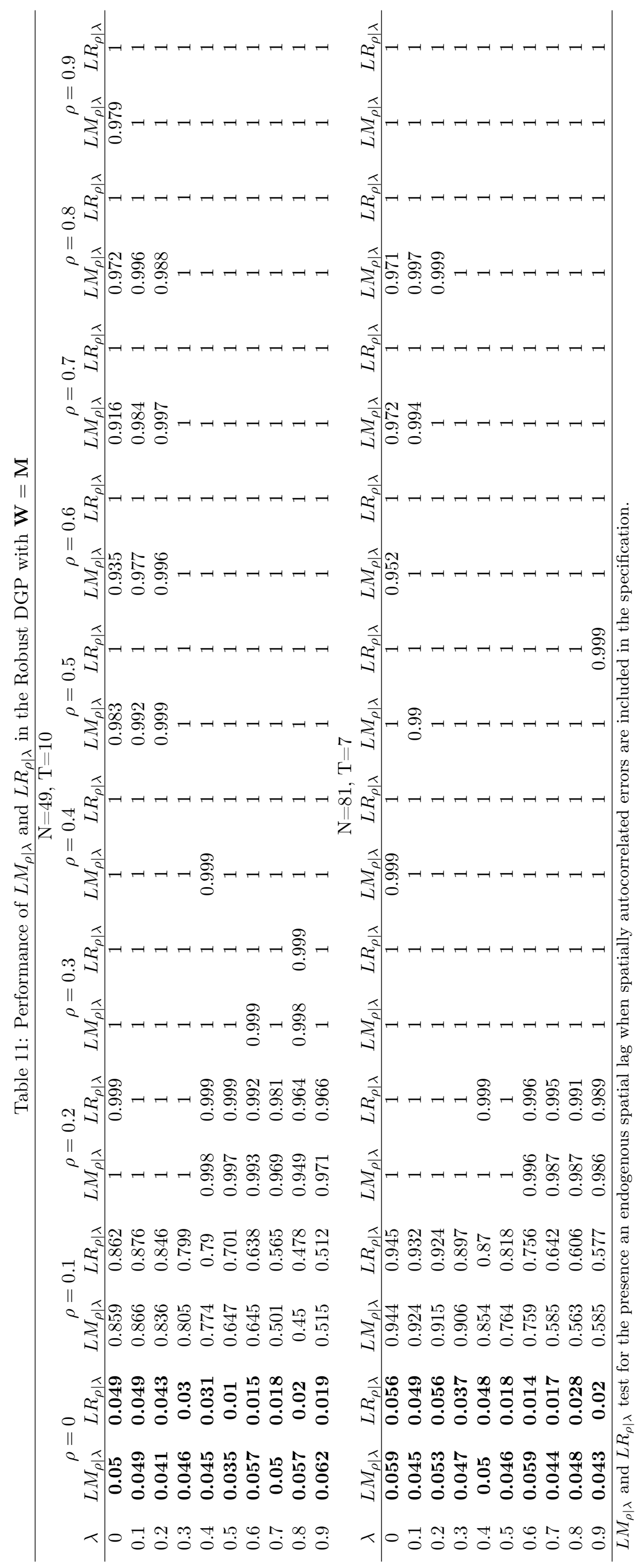


Table 12: Descriptive statistics for ratio of investment and saving

\begin{tabular}{cccccc}
\hline & Obs. & mean & Std deviation & $\min$ & $\max$ \\
Investment & 984 & 25.54793 & 5.987409 & 9.798207 & 44.85361 \\
Savings & 984 & 24.84542 & 6.990893 & 0.1976267 & 41.84594 \\
\hline
\end{tabular}

Table 13: Results of the within estimation Dependent variable Investment rate

\begin{tabular}{cccc} 
Period & $1960-1970$ & $1971-1985$ & $1986-2000$ \\
\hline Saving rate & 0.932 & 0.707 & 0.442 \\
& $(0.000)$ & $(0.000)$ & $(0.000)$ \\
\hline$R^{2}$ & 0.653 & 0.453 & 0.108 \\
\hline
\end{tabular}

p-values are between brackets. 
Table 14: Tests to detect spatial autocorrelation

$1960-1970 \quad 1971-1985 \quad 1986-2000$

Weight matrix : inverse distance

\begin{tabular}{|c|c|c|c|}
\hline$L M_{J}$ & $\begin{array}{c}2.03 \\
(0.363)\end{array}$ & $\begin{array}{l}166.97 \\
(0.000)\end{array}$ & $\begin{array}{c}82.58 \\
(0.000)\end{array}$ \\
\hline$L R_{J}$ & $\begin{array}{c}2.50 \\
(0.286)\end{array}$ & $\begin{array}{c}73.36 \\
(0.000)\end{array}$ & $\begin{array}{c}43.08 \\
(0.000)\end{array}$ \\
\hline$L M_{\lambda}$ & - & $\begin{array}{l}147.51 \\
(0.000)\end{array}$ & $\begin{array}{c}72.79 \\
(0.000)\end{array}$ \\
\hline$L R_{\lambda}$ & - & $\begin{array}{c}70.47 \\
(0.000)\end{array}$ & $\begin{array}{c}39.07 \\
(0.000)\end{array}$ \\
\hline$L M_{\rho}$ & - & $\begin{array}{l}133.53 \\
(0.000)\end{array}$ & $\begin{array}{l}82.56 \\
(0.000)\end{array}$ \\
\hline$L R_{\rho}$ & - & $\begin{array}{c}69.86 \\
(0.000)\end{array}$ & $\begin{array}{l}42.08 \\
(0.000)\end{array}$ \\
\hline$L M_{\lambda \mid \rho}$ & - & $\begin{array}{c}2.14 \\
(0.143)\end{array}$ & $\begin{array}{c}0.44 \\
(0.504)\end{array}$ \\
\hline$L R_{\lambda \mid \rho}$ & - & $\begin{array}{c}3.49 \\
(0.062)\end{array}$ & $\begin{array}{c}1.06 \\
(0.316)\end{array}$ \\
\hline$L M_{\rho \mid \lambda}$ & - & $\begin{array}{l}100.52 \\
(0.000)\end{array}$ & $\begin{array}{l}183.64 \\
(0.000)\end{array}$ \\
\hline$L R_{\rho \mid \lambda}$ & - & $\begin{array}{c}2.88 \\
(0.089)\end{array}$ & $\begin{array}{c}4.01 \\
(0.045)\end{array}$ \\
\hline Preferred model & a-spatial & SAR & SAR \\
\hline $\begin{array}{c}\text { Test for } \\
\text { individual effects }\end{array}$ & $\begin{array}{c}26.44 \\
(0.000) \\
\end{array}$ & $\begin{array}{l}234.59 \\
(0.000) \\
\end{array}$ & $\begin{array}{l}291.62 \\
(0.000) \\
\end{array}$ \\
\hline \multicolumn{4}{|c|}{ "Weight matrix : 7 Nearest neighbours } \\
\hline$L M_{J}$ & $\begin{array}{c}2.54 \\
(0.280)\end{array}$ & $\begin{array}{l}128.61 \\
(0.000)\end{array}$ & $\begin{array}{c}56.40 \\
(0.000)\end{array}$ \\
\hline$L R_{J}$ & $\begin{array}{c}3.27 \\
(0.194)\end{array}$ & $\begin{array}{c}64.12 \\
(0.000)\end{array}$ & $\begin{array}{l}33.10 \\
(0.000)\end{array}$ \\
\hline$L M_{\lambda}$ & - & $\begin{array}{l}114.03 \\
(0.000)\end{array}$ & $\begin{array}{c}52.69 \\
(0.000)\end{array}$ \\
\hline$L R_{\lambda}$ & - & $\begin{array}{c}60.93 \\
(0.000)\end{array}$ & $\begin{array}{c}30.43 \\
(0.000)\end{array}$ \\
\hline$L M_{\rho}$ & - & $\begin{array}{l}105.75 \\
(0.000)\end{array}$ & $\begin{array}{c}55.73 \\
(0.000)\end{array}$ \\
\hline$L R_{\rho}$ & - & $\begin{array}{c}61.65 \\
(0.000)\end{array}$ & $\begin{array}{c}33.10 \\
(0.000)\end{array}$ \\
\hline$L M_{\lambda \mid \rho}$ & - & $\begin{array}{c}1.37 \\
(0.241)\end{array}$ & $\begin{array}{c}0.01 \\
(0.965)\end{array}$ \\
\hline$L R_{\lambda \mid \rho}$ & - & $\begin{array}{c}2.46 \\
(0.116)\end{array}$ & $\begin{array}{c}0.01 \\
(0.931)\end{array}$ \\
\hline$L M_{\rho \mid \lambda}$ & & $\begin{array}{l}106.48 \\
(0.000)\end{array}$ & $\begin{array}{l}154.44 \\
(0.000)\end{array}$ \\
\hline$L R_{\rho \mid \lambda}$ & - & $\begin{array}{c}3.18 \\
(0.074)\end{array}$ & $\begin{array}{c}2.68 \\
(0.102)\end{array}$ \\
\hline Preferred model & a-spatial & SAR & SAR \\
\hline $\begin{array}{c}\text { Test for } \\
\text { individual effects }\end{array}$ & $\begin{array}{c}26.44 \\
(0.000)\end{array}$ & $\begin{array}{l}226.63 \\
(0.000)\end{array}$ & $\begin{array}{l}303.44 \\
(0.000)\end{array}$ \\
\hline
\end{tabular}


Table 15: Estimation of final models

\begin{tabular}{|c|c|c|c|}
\hline \multicolumn{4}{|c|}{ Dependent variable : Investment } \\
\hline & 1960-1970 & 1971-1985 & 1986-2000 \\
\hline \multicolumn{4}{|c|}{ Weight matrix : inverse distance } \\
\hline Model & a-spatial & SAR & SAR \\
\hline \multirow{2}{*}{ Savings } & 0.932 & 0.614 & 0.355 \\
\hline & $(0.000)$ & $(0.000)$ & $(0.000)$ \\
\hline \multirow[t]{2}{*}{$\rho$} & & 0.507 & 0.535 \\
\hline & & $(0.000)$ & $(0.000)$ \\
\hline Tests for & 26.440 & 234.59 & 291.62 \\
\hline fixed effects & $(0.000)$ & $(0.000)$ & $(0.000)$ \\
\hline \multicolumn{4}{|c|}{ Weight matrix : 7 nearest neighbours } \\
\hline Model & a-spatial & SAR & SAR \\
\hline \multirow[t]{2}{*}{ Savings } & 0.932 & 0.609 & 0.367 \\
\hline & $(0.000)$ & $(0.000)$ & $(0.000)$ \\
\hline \multirow[t]{2}{*}{$\rho$} & & 0.452 & 0.451 \\
\hline & & $(0.000)$ & $(0.000)$ \\
\hline Tests for & 26.440 & 226.63 & 303.44 \\
\hline fixed effects & $(0.000)$ & $(0.000)$ & $(0.000)$ \\
\hline
\end{tabular}

p-values are between brackets. $\rho$ is the spatial autoregressive parameter associated with the spatial endogenous lag. Tests for fixed effects are the usual Chow test for the a-spatial model and LR tests for the last two subsamples since the Chow statistic is affected by the presence of spatial autocorrelation. 
Table 16 : Matrix of reaction coefficients for the 1971-1985 period.

\begin{tabular}{lcccccccc}
\hline & AUS & AUT & BEL & CAN & CHE & DNK & ESP & FIN \\
AUS & $\mathbf{0 . 6 2 5 1}$ & 0.0254 & 0.0327 & 0.019 & 0.0274 & 0.0274 & 0.022 & 0.0243 \\
AUT & 0.0034 & $\mathbf{0 . 6 3 1 6}$ & 0.0528 & 0.0086 & 0.0523 & 0.0443 & 0.0256 & 0.0292 \\
BEL & 0.0025 & 0.0299 & $\mathbf{0 . 6 5 0 2}$ & 0.0069 & 0.0464 & 0.0353 & 0.0226 & 0.0205 \\
CAN & 0.0074 & 0.0249 & 0.0354 & $\mathbf{0 . 6 3 2 2}$ & 0.0285 & 0.0284 & 0.0246 & 0.0236 \\
CHE & 0.003 & 0.0422 & 0.0661 & 0.0079 & $\mathbf{0 . 6 3 6}$ & 0.0361 & 0.0288 & 0.0229 \\
DNK & 0.0032 & 0.0377 & 0.0532 & 0.0083 & 0.0381 & $\mathbf{0 . 6 3 5 6}$ & 0.0219 & 0.0365 \\
ESP & 0.0037 & 0.0315 & 0.0492 & 0.0105 & 0.0439 & 0.0316 & $\mathbf{0 . 6 3 1 1}$ & 0.0224 \\
FIN & 0.0039 & 0.0344 & 0.0426 & 0.0096 & 0.0334 & 0.0504 & 0.0214 & $\mathbf{0 . 6 3 1 8}$ \\
FRA & 0.0027 & 0.0305 & 0.0844 & 0.0074 & 0.0536 & 0.033 & 0.0271 & 0.0205 \\
GBR & 0.0027 & 0.0285 & 0.079 & 0.0077 & 0.0423 & 0.0342 & 0.0256 & 0.0211 \\
GRC & 0.0046 & 0.0438 & 0.0437 & 0.0101 & 0.0413 & 0.0352 & 0.0281 & 0.028 \\
IRL & 0.0033 & 0.0294 & 0.0591 & 0.0099 & 0.0391 & 0.037 & 0.0287 & 0.0245 \\
ISL & 0.0048 & 0.0303 & 0.0469 & 0.017 & 0.0353 & 0.0387 & 0.0275 & 0.031 \\
ITA & 0.0036 & 0.0491 & 0.0501 & 0.0091 & 0.0563 & 0.0355 & 0.0319 & 0.0251 \\
JPN & 0.0174 & 0.0261 & 0.0338 & 0.0175 & 0.0279 & 0.0289 & 0.0218 & 0.0264 \\
KOR & 0.0161 & 0.0267 & 0.0344 & 0.0167 & 0.0285 & 0.0296 & 0.0221 & 0.0272 \\
MEX & 0.0126 & 0.0266 & 0.0369 & 0.0425 & 0.0301 & 0.0298 & 0.0259 & 0.0251 \\
NLD & 0.0026 & 0.0306 & 0.1046 & 0.0072 & 0.0426 & 0.0405 & 0.0222 & 0.0222 \\
NOR & 0.0034 & 0.0321 & 0.0471 & 0.009 & 0.034 & 0.0628 & 0.0214 & 0.0424 \\
NZL & 0.0782 & 0.0247 & 0.0321 & 0.0211 & 0.0267 & 0.0268 & 0.0215 & 0.0237 \\
PRT & 0.004 & 0.0304 & 0.0469 & 0.0118 & 0.0402 & 0.0314 & 0.0755 & 0.0227 \\
SWE & 0.0034 & 0.0332 & 0.0433 & 0.0087 & 0.0329 & 0.06 & 0.0205 & 0.0647 \\
TUR & 0.0051 & 0.0418 & 0.0427 & 0.0106 & 0.0385 & 0.0365 & 0.0264 & 0.0312 \\
USA & 0.0077 & 0.0245 & 0.0347 & 0.0979 & 0.0281 & 0.0277 & 0.0244 & 0.023 \\
\hline
\end{tabular}


Table 16 (continued)

\begin{tabular}{ccccccccc}
\hline & FRA & GBR & GRC & IRL & ISL & ITA & JPN & KOR \\
AUS & 0.0299 & 0.0294 & 0.0218 & 0.0241 & 0.0194 & 0.0237 & 0.0291 & 0.0282 \\
AUT & 0.0461 & 0.0419 & 0.0281 & 0.0292 & 0.0164 & 0.0432 & 0.0059 & 0.0063 \\
BEL & 0.0722 & 0.0656 & 0.0159 & 0.0332 & 0.0144 & 0.0249 & 0.0043 & 0.0046 \\
CAN & 0.0325 & 0.0327 & 0.0187 & 0.0285 & 0.0266 & 0.0231 & 0.0114 & 0.0115 \\
CHE & 0.0653 & 0.05 & 0.0214 & 0.0312 & 0.0154 & 0.0399 & 0.0051 & 0.0054 \\
DNK & 0.0425 & 0.0427 & 0.0193 & 0.0312 & 0.0179 & 0.0265 & 0.0056 & 0.0059 \\
ESP & 0.0505 & 0.0464 & 0.0222 & 0.0351 & 0.0183 & 0.0346 & 0.0061 & 0.0064 \\
FIN & 0.0365 & 0.0364 & 0.0212 & 0.0286 & 0.0198 & 0.026 & 0.007 & 0.0076 \\
FRA & $\mathbf{0 . 6 4 2 8}$ & 0.0725 & 0.017 & 0.0356 & 0.0151 & 0.0276 & 0.0046 & 0.0049 \\
GBR & 0.0746 & $\mathbf{0 . 6 4 1 9}$ & 0.0163 & 0.0463 & 0.0164 & 0.025 & 0.0047 & 0.005 \\
GRC & 0.0401 & 0.0373 & $\mathbf{0 . 6 2 7 2}$ & 0.0278 & 0.0171 & 0.0473 & 0.0075 & 0.0081 \\
IRL & 0.0542 & 0.0686 & 0.018 & $\mathbf{0 . 6 3 1 2}$ & 0.0234 & 0.0261 & 0.0057 & 0.006 \\
ISL & 0.0421 & 0.0444 & 0.0203 & 0.0427 & $\mathbf{0 . 6 2 3 1}$ & 0.0266 & 0.0086 & 0.009 \\
ITA & 0.0475 & 0.0418 & 0.0345 & 0.0294 & 0.0164 & $\mathbf{0 . 6 3}$ & 0.0061 & 0.0065 \\
JPN & 0.0306 & 0.0303 & 0.0214 & 0.025 & 0.0207 & 0.0237 & $\mathbf{0 . 6 2 8 6}$ & 0.0887 \\
KOR & 0.0311 & 0.0308 & 0.022 & 0.0253 & 0.0207 & 0.0242 & 0.0849 & $\mathbf{0 . 6 2 8 6}$ \\
MEX & 0.0339 & 0.0338 & 0.0208 & 0.0288 & 0.025 & 0.0249 & 0.0157 & 0.0153 \\
NLD & 0.0585 & 0.0618 & 0.0162 & 0.0346 & 0.0152 & 0.0246 & 0.0045 & 0.0048 \\
NOR & 0.0391 & 0.0405 & 0.0185 & 0.0323 & 0.0208 & 0.0245 & 0.006 & 0.0064 \\
NZL & 0.0292 & 0.0289 & 0.021 & 0.0239 & 0.0197 & 0.023 & 0.0274 & 0.0263 \\
PRT & 0.0467 & 0.0446 & 0.022 & 0.0357 & 0.0198 & 0.0321 & 0.0065 & 0.0069 \\
SWE & 0.0364 & 0.0367 & 0.0192 & 0.0286 & 0.0188 & 0.0246 & 0.0061 & 0.0065 \\
TUR & 0.0386 & 0.0366 & 0.0583 & 0.0277 & 0.0179 & 0.0385 & 0.0086 & 0.0093 \\
USA & 0.0319 & 0.032 & 0.0186 & 0.0277 & 0.0252 & 0.0229 & 0.0115 & 0.0115 \\
\hline
\end{tabular}


Table 16 (continued)

\begin{tabular}{ccccccccc}
\hline & MEX & NLD & NOR & NZL & PRT & SWE & TUR & USA \\
AUS & 0.0181 & 0.0313 & 0.0262 & 0.0734 & 0.0202 & 0.0269 & 0.0212 & 0.0189 \\
AUT & 0.0052 & 0.0498 & 0.0339 & 0.0031 & 0.0209 & 0.0356 & 0.0233 & 0.0081 \\
BEL & 0.004 & 0.0963 & 0.0282 & 0.0023 & 0.0183 & 0.0263 & 0.0135 & 0.0065 \\
CAN & 0.0239 & 0.0338 & 0.0277 & 0.0078 & 0.0236 & 0.0272 & 0.0171 & 0.0936 \\
CHE & 0.0047 & 0.0558 & 0.029 & 0.0027 & 0.0224 & 0.0284 & 0.0173 & 0.0075 \\
DNK & 0.0049 & 0.0561 & 0.0565 & 0.0029 & 0.0184 & 0.0547 & 0.0173 & 0.0078 \\
ESP & 0.0062 & 0.0445 & 0.0279 & 0.0034 & 0.0642 & 0.0271 & 0.0181 & 0.0099 \\
FIN & 0.0057 & 0.0425 & 0.0528 & 0.0035 & 0.0185 & 0.0817 & 0.0205 & 0.009 \\
FRA & 0.0044 & 0.063 & 0.0274 & 0.0025 & 0.0213 & 0.0258 & 0.0142 & 0.007 \\
GBR & 0.0045 & 0.0685 & 0.0292 & 0.0025 & 0.0209 & 0.0268 & 0.0139 & 0.0072 \\
GRC & 0.0063 & 0.041 & 0.0304 & 0.0042 & 0.0237 & 0.032 & 0.0506 & 0.0096 \\
IRL & 0.0056 & 0.0568 & 0.0345 & 0.0031 & 0.0249 & 0.0309 & 0.0156 & 0.0092 \\
ISL & 0.009 & 0.0458 & 0.0406 & 0.0046 & 0.0251 & 0.0372 & 0.0184 & 0.0153 \\
ITA & 0.0055 & 0.0456 & 0.0295 & 0.0033 & 0.0252 & 0.03 & 0.0244 & 0.0086 \\
JPN & 0.0135 & 0.0325 & 0.0281 & 0.0154 & 0.0199 & 0.0289 & 0.0211 & 0.0167 \\
KOR & 0.0126 & 0.033 & 0.0286 & 0.0141 & 0.0201 & 0.0296 & 0.022 & 0.016 \\
MEX & $\mathbf{0 . 6 1 9 5}$ & 0.0352 & 0.0288 & 0.0139 & 0.0247 & 0.0286 & 0.0191 & 0.0472 \\
NLD & 0.0042 & $\mathbf{0 . 6 4 6 2}$ & 0.0316 & 0.0024 & 0.0182 & 0.0289 & 0.0139 & 0.0067 \\
NOR & 0.0053 & 0.0487 & $\mathbf{0 . 6 3 5}$ & 0.0031 & 0.0184 & 0.0685 & 0.0169 & 0.0084 \\
NZL & 0.0212 & 0.0307 & 0.0258 & $\mathbf{0 . 6 2 5}$ & 0.0201 & 0.0263 & 0.0203 & 0.0212 \\
PRT & 0.0069 & 0.0429 & 0.0282 & 0.0037 & $\mathbf{0 . 6 2 8 9}$ & 0.0273 & 0.0182 & 0.0112 \\
SWE & 0.0052 & 0.0439 & 0.0676 & 0.0031 & 0.0176 & $\mathbf{0 . 6 3 7 8}$ & 0.0179 & 0.0082 \\
TUR & 0.0067 & 0.0405 & 0.0321 & 0.0046 & 0.0226 & 0.0345 & $\mathbf{0 . 6 2 5 4}$ & 0.01 \\
USA & 0.0278 & 0.0331 & 0.027 & 0.0082 & 0.0234 & 0.0265 & 0.017 & $\mathbf{0 . 6 3 2 4}$ \\
\hline AII & & & & & & &
\end{tabular}

All numbers are significant at the $1 \%$ level. 
Table 17 : Matrix of reaction coefficients for the 1986-2000 period.

\begin{tabular}{ccccccccc}
\hline & AUS & AUT & BEL & CAN & CHE & DNK & ESP & FIN \\
AUS & $\mathbf{0 . 3 6 2 1}$ & 0.0166 & 0.0218 & 0.0121 & 0.0181 & 0.018 & 0.0144 & 0.0158 \\
AUT & 0.0022 & $\mathbf{0 . 3 6 6 8}$ & 0.0343 & 0.0056 & 0.0334 & 0.0284 & 0.0166 & 0.0188 \\
BEL & 0.0017 & 0.0194 & $\mathbf{0 . 3 7 9 3}$ & 0.0046 & 0.0298 & 0.0229 & 0.0148 & 0.0135 \\
CAN & 0.0048 & 0.0163 & 0.0235 & $\mathbf{0 . 3 6 6 8}$ & 0.0188 & 0.0186 & 0.016 & 0.0154 \\
CHE & 0.002 & 0.0269 & 0.0425 & 0.0052 & $\mathbf{0 . 3 6 9 8}$ & 0.0234 & 0.0185 & 0.0149 \\
DNK & 0.0021 & 0.0242 & 0.0345 & 0.0055 & 0.0247 & $\mathbf{0 . 3 6 9 5}$ & 0.0143 & 0.0233 \\
ESP & 0.0024 & 0.0204 & 0.0321 & 0.0068 & 0.0283 & 0.0207 & $\mathbf{0 . 3 6 6 3}$ & 0.0147 \\
FIN & 0.0025 & 0.0222 & 0.028 & 0.0062 & 0.0218 & 0.0322 & 0.014 & $\mathbf{0 . 3 6 6 9}$ \\
FRA & 0.0018 & 0.0198 & 0.0536 & 0.0049 & 0.0342 & 0.0215 & 0.0175 & 0.0135 \\
GBR & 0.0018 & 0.0186 & 0.0504 & 0.0051 & 0.0273 & 0.0222 & 0.0166 & 0.0138 \\
GRC & 0.003 & 0.0279 & 0.0287 & 0.0066 & 0.0267 & 0.0229 & 0.0181 & 0.0181 \\
IRL & 0.0022 & 0.0191 & 0.0382 & 0.0064 & 0.0253 & 0.024 & 0.0185 & 0.0159 \\
ISL & 0.0031 & 0.0197 & 0.0307 & 0.0108 & 0.023 & 0.025 & 0.0177 & 0.0199 \\
ITA & 0.0024 & 0.0311 & 0.0326 & 0.0059 & 0.0358 & 0.023 & 0.0204 & 0.0163 \\
JPN & 0.0109 & 0.0171 & 0.0225 & 0.0112 & 0.0184 & 0.019 & 0.0142 & 0.0171 \\
KOR & 0.0101 & 0.0174 & 0.0228 & 0.0107 & 0.0187 & 0.0194 & 0.0144 & 0.0176 \\
MEX & 0.0079 & 0.0174 & 0.0244 & 0.0266 & 0.0198 & 0.0195 & 0.0168 & 0.0163 \\
NLD & 0.0017 & 0.0199 & 0.0658 & 0.0047 & 0.0275 & 0.0261 & 0.0145 & 0.0145 \\
NOR & 0.0022 & 0.0208 & 0.0308 & 0.0059 & 0.0222 & 0.0397 & 0.014 & 0.0269 \\
NZL & 0.0479 & 0.0162 & 0.0214 & 0.0134 & 0.0176 & 0.0177 & 0.0141 & 0.0155 \\
PRT & 0.0026 & 0.0197 & 0.0307 & 0.0076 & 0.026 & 0.0205 & 0.047 & 0.0148 \\
SWE & 0.0022 & 0.0215 & 0.0284 & 0.0057 & 0.0215 & 0.038 & 0.0134 & 0.0405 \\
TUR & 0.0033 & 0.0267 & 0.028 & 0.0069 & 0.025 & 0.0236 & 0.0171 & 0.02 \\
USA & 0.0049 & 0.0161 & 0.023 & 0.0603 & 0.0185 & 0.0182 & 0.0158 & 0.015 \\
\hline & & & & & & & & \\
\hline
\end{tabular}


Table 17 (continued)

\begin{tabular}{lcccccccc}
\hline & FRA & GBR & GRC & IRL & ISL & ITA & JPN & KOR \\
AUS & 0.0198 & 0.0195 & 0.0141 & 0.0158 & 0.0125 & 0.0155 & 0.0182 & 0.0177 \\
AUT & 0.0299 & 0.0273 & 0.0179 & 0.019 & 0.0106 & 0.0274 & 0.0039 & 0.0041 \\
BEL & 0.0458 & 0.0418 & 0.0104 & 0.0214 & 0.0094 & 0.0162 & 0.0029 & 0.0031 \\
CAN & 0.0215 & 0.0216 & 0.0122 & 0.0185 & 0.0169 & 0.0151 & 0.0073 & 0.0073 \\
CHE & 0.0416 & 0.0323 & 0.0138 & 0.0202 & 0.01 & 0.0254 & 0.0034 & 0.0036 \\
DNK & 0.0277 & 0.0278 & 0.0125 & 0.0202 & 0.0115 & 0.0172 & 0.0037 & 0.0039 \\
ESP & 0.0326 & 0.03 & 0.0143 & 0.0226 & 0.0118 & 0.0221 & 0.004 & 0.0042 \\
FIN & 0.024 & 0.0239 & 0.0137 & 0.0186 & 0.0127 & 0.0169 & 0.0046 & 0.0049 \\
FRA & $\mathbf{0 . 3 7 4 3}$ & 0.046 & 0.0111 & 0.0229 & 0.0098 & 0.0179 & 0.003 & 0.0032 \\
GBR & 0.0473 & $\mathbf{0 . 3 7 3 7}$ & 0.0107 & 0.0294 & 0.0106 & 0.0163 & 0.0031 & 0.0033 \\
GRC & 0.0262 & 0.0244 & $\mathbf{0 . 3 6 3 8}$ & 0.0181 & 0.0111 & 0.0299 & 0.0049 & 0.0052 \\
IRL & 0.0349 & 0.0436 & 0.0117 & $\mathbf{0 . 3 6 6 5}$ & 0.0149 & 0.0169 & 0.0037 & 0.0039 \\
ISL & 0.0274 & 0.0288 & 0.0132 & 0.0272 & $\mathbf{0 . 3 6 1 1}$ & 0.0172 & 0.0056 & 0.0058 \\
ITA & 0.0307 & 0.0272 & 0.0218 & 0.0191 & 0.0106 & $\mathbf{0 . 3 6 5 7}$ & 0.004 & 0.0042 \\
JPN & 0.0203 & 0.0201 & 0.0138 & 0.0164 & 0.0133 & 0.0155 & $\mathbf{0 . 3 6 4 4}$ & 0.0545 \\
KOR & 0.0206 & 0.0203 & 0.0142 & 0.0165 & 0.0133 & 0.0158 & 0.0521 & $\mathbf{0 . 3 6 4 4}$ \\
MEX & 0.0223 & 0.0223 & 0.0134 & 0.0187 & 0.0159 & 0.0162 & 0.0099 & 0.0097 \\
NLD & 0.0376 & 0.0395 & 0.0106 & 0.0223 & 0.0099 & 0.0161 & 0.003 & 0.0032 \\
NOR & 0.0256 & 0.0264 & 0.012 & 0.0209 & 0.0133 & 0.016 & 0.0039 & 0.0042 \\
NZL & 0.0194 & 0.0192 & 0.0136 & 0.0157 & 0.0127 & 0.015 & 0.0172 & 0.0165 \\
PRT & 0.0303 & 0.0289 & 0.0142 & 0.023 & 0.0127 & 0.0206 & 0.0042 & 0.0045 \\
SWE & 0.0239 & 0.0241 & 0.0124 & 0.0186 & 0.0121 & 0.016 & 0.004 & 0.0043 \\
TUR & 0.0253 & 0.024 & 0.0363 & 0.0181 & 0.0116 & 0.0246 & 0.0055 & 0.006 \\
USA & 0.0211 & 0.0211 & 0.0121 & 0.0181 & 0.0161 & 0.0149 & 0.0074 & 0.0074 \\
\hline & & & & & & & & \\
\hline
\end{tabular}


Table 17 (continued)

\begin{tabular}{ccccccccc}
\hline & MEX & NLD & NOR & NZL & PRT & SWE & TUR & USA \\
AUS & 0.0114 & 0.0208 & 0.0172 & 0.045 & 0.0131 & 0.0176 & 0.0136 & 0.0121 \\
AUT & 0.0034 & 0.0323 & 0.0219 & 0.0021 & 0.0136 & 0.023 & 0.0149 & 0.0053 \\
BEL & 0.0027 & 0.0606 & 0.0184 & 0.0015 & 0.012 & 0.0173 & 0.0088 & 0.0043 \\
CAN & 0.0149 & 0.0224 & 0.0181 & 0.0049 & 0.0152 & 0.0178 & 0.0111 & 0.0576 \\
CHE & 0.0031 & 0.036 & 0.0189 & 0.0018 & 0.0145 & 0.0186 & 0.0112 & 0.0049 \\
DNK & 0.0032 & 0.0361 & 0.0358 & 0.0019 & 0.012 & 0.0347 & 0.0112 & 0.0051 \\
ESP & 0.004 & 0.029 & 0.0182 & 0.0022 & 0.0399 & 0.0178 & 0.0117 & 0.0064 \\
FIN & 0.0037 & 0.0278 & 0.0335 & 0.0023 & 0.0121 & 0.0511 & 0.0132 & 0.0058 \\
FRA & 0.0029 & 0.0405 & 0.0179 & 0.0016 & 0.0138 & 0.017 & 0.0093 & 0.0046 \\
GBR & 0.0029 & 0.0438 & 0.019 & 0.0017 & 0.0136 & 0.0176 & 0.0091 & 0.0047 \\
GRC & 0.0041 & 0.0269 & 0.0198 & 0.0027 & 0.0153 & 0.0208 & 0.0315 & 0.0062 \\
IRL & 0.0037 & 0.0366 & 0.0223 & 0.002 & 0.016 & 0.0201 & 0.0102 & 0.006 \\
ISL & 0.0057 & 0.0298 & 0.026 & 0.003 & 0.0162 & 0.024 & 0.0119 & 0.0098 \\
ITA & 0.0036 & 0.0297 & 0.0192 & 0.0022 & 0.0162 & 0.0196 & 0.0156 & 0.0056 \\
JPN & 0.0085 & 0.0215 & 0.0183 & 0.0097 & 0.0129 & 0.0189 & 0.0136 & 0.0107 \\
KOR & 0.008 & 0.0219 & 0.0187 & 0.0089 & 0.013 & 0.0193 & 0.0141 & 0.0103 \\
MEX & $\mathbf{0 . 3 5 8 5}$ & 0.0232 & 0.0188 & 0.0087 & 0.0159 & 0.0187 & 0.0123 & 0.0294 \\
NLD & 0.0028 & $\mathbf{0 . 3 7 6 6}$ & 0.0205 & 0.0016 & 0.0119 & 0.0189 & 0.0091 & 0.0044 \\
NOR & 0.0034 & 0.0316 & $\mathbf{0 . 3 6 9}$ & 0.002 & 0.012 & 0.0431 & 0.011 & 0.0055 \\
NZL & 0.0132 & 0.0204 & 0.0169 & $\mathbf{0 . 3 6 2}$ & 0.0131 & 0.0173 & 0.0131 & 0.0135 \\
PRT & 0.0045 & 0.0281 & 0.0184 & 0.0024 & $\mathbf{0 . 3 6 4 9}$ & 0.0179 & 0.0118 & 0.0072 \\
SWE & 0.0034 & 0.0287 & 0.0425 & 0.002 & 0.0115 & $\mathbf{0 . 3 7 0 8}$ & 0.0116 & 0.0054 \\
TUR & 0.0043 & 0.0266 & 0.0208 & 0.003 & 0.0146 & 0.0223 & $\mathbf{0 . 3 6 2 6}$ & 0.0065 \\
USA & 0.0173 & 0.0219 & 0.0176 & 0.0052 & 0.0151 & 0.0174 & 0.011 & $\mathbf{0 . 3 6 6 9}$ \\
\hline All & &
\end{tabular}

All numbers are significant at the $1 \%$ level. 